\title{
Nutritional immunity: the impact of metals on lung immune cells and the airway microbiome during chronic respiratory disease
}

\author{
Claire Healy ${ }^{1,2}$, Natalia Munoz-Wolf ${ }^{1,2}$, Janné Strydom,2, Lynne Faherty ${ }^{1,2}$, Niamh C. Williams ${ }^{1,2}$, Sarah Kenny ${ }^{1}$, \\ Seamas C. Donnelly, ${ }^{1,2}$ and Suzanne M. Cloonan ${ }^{1,2,3^{*}} \mathbb{0}$
}

\begin{abstract}
Nutritional immunity is the sequestration of bioavailable trace metals such as iron, zinc and copper by the host to limit pathogenicity by invading microorganisms. As one of the most conserved activities of the innate immune system, limiting the availability of free trace metals by cells of the immune system serves not only to conceal these vital nutrients from invading bacteria but also operates to tightly regulate host immune cell responses and function. In the setting of chronic lung disease, the regulation of trace metals by the host is often disrupted, leading to the altered availability of these nutrients to commensal and invading opportunistic pathogenic microbes. Similarly, alterations in the uptake, secretion, turnover and redox activity of these vitally important metals has significant repercussions for immune cell function including the response to and resolution of infection. This review will discuss the intricate role of nutritional immunity in host immune cells of the lung and how changes in this fundamental process as a result of chronic lung disease may alter the airway microbiome, disease progression and the response to infection.
\end{abstract}

Keywords: Nutritional immunity, Microbiome, Immunity, Metals, Iron, COPD, Asthma, IPF, Mycobacteria

\section{Background}

Respiratory disease remains a global leading cause of death despite the advancements made by respiratory research [1]. Furthermore, those with poor respiratory health have a greatly diminished quality of life and recurrent hospitalizations. To alleviate the immense burden of respiratory disease we need a greater understanding of the resident and recruited immune cells in the lungs, their role in respiratory disease, and their complex interplay with the lung microbiome and with invading pathogenic microorganisms. Metabolite and nutrient availability in the lung, whether produced by the host or the microbiota, are likely to have a crucial role in the progression of

*Correspondence: suzanne.cloonan@tcd.ie

${ }^{1}$ School of Medicine, Trinity Biomedical Sciences Institute, Trinity College Dublin, Dublin, Ireland

Full list of author information is available at the end of the article lung disease as many immune cells require these specific nutrients to fuel their immune function when activated.

Nutritional immunity is classically defined as the sequestration of trace metals, most notably iron, by the host organism in an effort to block bacterial metal acquisition and thus limit disease progression during infection. Trace metals are essential to all forms of life. Most organisms require manganese, cobalt, iron, copper, nickel, selenium and zinc. Metal cofactors serve both catalytic and structural roles in a range of biological processes. With the exception of zinc, these metals are redox-active and this property contributes both to their catalytic activities and toxicity. Iron is the most abundant element on Earth and is also the most widely used metal in biological processes. This dependence on iron for most organisms makes it a vital currency in the context of host-pathogen interactions. The host takes advantage of this by original author(s) and the source, provide a link to the Creative Commons licence, and indicate if changes were made. The images or other third party material in this article are included in the article's Creative Commons licence, unless indicated otherwise in a credit line to the material. If material is not included in the article's Creative Commons licence and your intended use is not permitted by statutory regulation or exceeds the permitted use, you will need to obtain permission directly from the copyright holder. To view a copy of this licence, visit http://creativecommons.org/licenses/by/4.0/. The Creative Commons Public Domain Dedication waiver (http://creativeco mmons.org/publicdomain/zero/1.0/) applies to the data made available in this article, unless otherwise stated in a credit line to the data. 
sequestering iron during infection as a means of blocking iron acquisition by the invading microorganism, whereas bacteria have evolved mechanisms to steal iron from the host despite host iron sequestration [2]. Many respiratory pathogens such as Mycobacterium tuberculosis, Pseudomonas aeruginosa, Klebsiella pneumoniae and Haemophilus influenzae require iron and use several iron acquisition strategies [3-6]. Similarly, the commensals that comprise the microbiome require iron and other metals to support their metabolism. Thus, iron homeostasis and iron sequestration in the lung is crucial to controlling lung infections and to support a normal microbiome [7, 8]. Though nutritional immunity was first described and best characterized for iron, the sequestration, and intoxication of other metals including, zinc, manganese and copper has also been described $[2,9]$.

While the concept of nutritional immunity has traditionally encompassed the sequestration of metals from microbes, the innate and adaptive immune systems can also actively utilize metals to facilitate bactericidal function. For example, the host utilizes copper to exert a bactericidal effect through both redox-dependent and independent mechanisms limiting bacterial growth and facilitating bacterial death [10-12]. Similarly, the S100 family of calcium-binding host proteins, primarily calprotectin, are commonly found at the site of infection, where they can chelate free zinc, iron, nickel, copper and manganese and exert bactericidal activity in addition to nutrient deprivation having a pleiotropic role in the nutritional immune response [13]. Less ubiquitous metals also have a role to play: selenium, as part of selenoproteins, is important in defence against viral replication, and host manganese can reduce superoxide dismutase (SOD) activity in pathogens [14]. The proper storage, metabolism, and utilisation of these metals by immune cells is therefore critical both in facilitating immune cell function and depriving microbes of the necessary nutrients for survival and proliferation in the host. Intriguingly, pathogen reliance on the acquisition of hostderived metal supports further investigations into therapeutic avenues for metal chelators in chronic and acute lung infection.

Finally, metabolites created through metal-dependent metabolic pathways are also essential for the function of immune cells such as macrophages, neutrophils and T-cells [15]. As our understanding of the rapidly emerging field of immunometabolism expands, we are now beginning to appreciate that trace metal biology may also be essential for the correct functioning of key metabolic pathways (e.g., glycolysis, fatty acid oxidation, the tricarboxylic acid cycle, etc.) engaged by immune cells. We could therefore expand the meaning of nutritional immunity to encompass not just the battle over trace metals but also the role of all metabolites and nutrients that are dependent on metal biology important for host pathogen interactions.

This review will discuss the key roles for metals in lung immune cell function, how metals are altered and dysregulated in chronic respiratory disease and the evidence if any, for the role of metals in dictating the repertoire of commensal bacteria present in the lung as well as the role of nutritional immunity in the response of the lung to infection.

\section{Metals, innate immune cells of the lung and response to infection}

Metal cofactors serve both catalytic and structural roles in a range of biological processes and play an important role in the development, maturation and function of immune cells. The role of metals in the biology of immune cells that are present in the lung is discussed below.

\section{Monocytes and macrophages}

Macrophages are a heterogenous family of professional phagocytes and are the most abundant immune cell present in the lung under homeostatic conditions [16]. Their key role in the respiratory immune response is highlighted by macrophage dysfunction contributing to chronic lung diseases including chronic obstructive pulmonary disease (COPD) [17], asthma [18] and cystic fibrosis [19]. Their phagocytic abilities make them excellent surveillance cells both in the context of lung homeostasis and inflammation or infection. Tissue resident macrophages maintain immune homeostasis by carrying out essential housekeeping roles such as tissue repair while also acting as a first line of defence against microbial infections [20]. There are two major classes of lung resident macrophage populations. Alveolar macrophages (AMs) are the most abundant and are easily characterised for their low levels of the phagocytic receptor CD11b, their autofluorescent nature and high levels of the integrin CD11c and the lectin SiglecF [21]. AMs originate from foetal liver cells in the embryonic yolk sac where under the influence of granulocyte macrophage-colony stimulating factor (GM-CSF) they remain and sustain themselves within the alveoli $[21,22]$. AMs reside within the lumen of the alveolus in close proximity to the alveolar epithelium and are directly exposed to air and the environment. AMs phagocytose antigens but also maintain homeostasis in the lung by catabolizing surfactant, removing particles as well as limiting inflammation [23, 24]. Originally thought to be sessile, AMs have recently been shown to be motile and to continuously crawl and cleanse all alveoli of particulate matter [25]. They also have the ability to communicate immunosuppressive 
signals to alveolar epithelial cells (AEC) [26]. AMs act as first line defence for respiratory pathogens, including bacteria and viruses such as Streptococcus pneumonia, Mycobacterium tuberculosis and influenza [27-29]. During infection AMs limit the inflammatory response by producing anti-inflammatory cytokines and by promoting tissue repair upon pathogen clearance [30, 31].

The second major resident lung macrophage population is the interstitial macrophage (IM) which is comprised of three phenotypically distinct subpopulations identified by their differential expression of CD11c, major histocompatibility complex (MHC)II and the mannose receptor CD206 [32, 33]. While their precise location in the lung remains controversial, IMs have been found in the lung parenchyma, both in the interstitium of the alveoli and the bronchovascular bundles with some specific subpopulations associated with nerves and blood vessels [32]. IMs originate and are maintained by circulating progenitor cells. They are smaller than AMs, immunoregulatory, and capable of antigen presentation [34].

In the setting of injury, inflammation, disease or with aging, resident AMs and IMs are often depleted and require the assistance of monocyte-derived macrophages that infiltrate the lung [35-38]. Once in the lung these infiltrating macrophages are defined by niche-derived tissue-instructive signals that trigger expression of specific differentiation programs, thus tailoring a particular lung specific functional identity [39].

\section{Macrophages, iron and heme}

Macrophages have been termed the "ferrostat" of tissue iron homeostasis [40]. Macrophages are vital for systemic iron homeostasis; supplying, sequestering or recycling iron as needed for erythropoiesis, bacteriostasis and erythrophagocytosis and constitute the main iron reservoir among immune cells and the third most important in the body after haemoglobin and liver ferritin stores [41]. Tissue-resident macrophages sequester and secrete iron on demand regulating local iron availability and modulating the tissue microenvironment, contributing to cellular and tissue function [40]. The exposure of AMs to a multitude of exogenous and endogenous sources in the lung, position AMs as key regulators of iron in the lung. As such, the storage, metabolism and detoxification of iron by AMs is paramount in their protection of alveoli against oxidative damage and maintenance of their innate immune functions (Table 1).

Following gut absorption of dietary and heme-conjugated iron, ferric iron $\left(\mathrm{Fe}^{3+}\right)$ is bound to the glycoprotein transferrin for systemic circulation. Uptake of iron by AMs is mediated through the iron transporters transferrin receptor 1 (TFR1) and divalent metal transporter 1 (DMT1); AMs also express the additional iron uptake proteins including low-density lipoprotein receptorrelated protein 1 (LRP1) and the zinc uptake receptor ZIP-14 (Fig. 1) [42-45]. Once inside the cell, iron dissociates from transferrin and is reduced to ferrous iron $\left(\mathrm{Fe}^{2+}\right)$ for storage in ferritin, a 'nanocage' like structure for safe storage of iron within the cytoplasm [46]. The transmembrane protein ferroportin (FPN) exports ferrous iron from AMs into the extracellular space, where it is oxidised and bound to transferrin in serum [42]. FPN is the only known exporter of elemental iron, allowing the release of iron into the circulation and to other cell types. In the lungs FPN is highly expressed in epithelial cells and AMs $[47,48]$. Decreased levels of FPN are observed in response to infection, in a bid to reduce cellular iron efflux and extracellular iron levels $[49,50]$. The systemic iron regulator hepcidin, expressed in response to proinflammatory cytokines and bone morphogenic protein 6 signalling, inhibits FPN to reduce circulating iron (Fig. 1) [51, 52]. While hepcidin does not seem to significantly contribute to AM lung iron trafficking [53], AMs produce endogenous hepcidin in response to challenge with the endotoxin lipopolysaccharide (LPS), potentially to sequester iron intracellularly through FPN degradation [53]. Indeed, this AM-produced hepcidin has proved essential for AM bactericidal function [54].

Heme (iron-protoporphyrin IX) is an essential metallocofactor and signalling molecule across all cell types. While in highest demand in erythroid cells, heme synthesis is an evolutionarily conserved process occurring in virtually all eukaryotic cell types. De novo heme synthesis is a multienzyme process which starts and culminates in the mitochondria, with the first step of glycine and succinyl CoA condensation by 5 -aminoevulinate synthase (ALAS) constituting the rate-limiting step in non-erythroid cells like AMs [55]. The hydrophobicity and cytotoxicity of heme necessitates carefully regulated handling in the cell, with such mechanisms of paramount importance in macrophages owing to their roles in erythrophagocytosis and heme degradation [56]. Unlike reticuloendothelial macrophages, which recycle iron from senescent erythrocytes via erythrophagocytosis [57] AMs in the alveoli do not seem to necessitate erythrophagocytosis at homeostasis. Instead, AMs uptake haemoglobin-haptoglobin and heme-hemopexin complexes via CD163 and CD63 respectively. However, AMs of patients exhibiting lung haemorrhage show iron overload, suggesting during increased heme burden AMs possess some erythophagocytic capability [58]. AMs play important roles regulating the iron pool by sensing free heme (the iron-containing porphyrin, key to $\mathrm{O}_{2}$ transport and storage among other biological processes) at concentrations reflective of pulmonary haemorrhage. AMs utilize heme to produce reactive oxygen species (ROS) and nitric oxide (NO) 
Table 1 Metal homeostasis within immune cells

\begin{tabular}{|c|c|c|c|}
\hline & Iron & Zinc & Copper \\
\hline Macrophages & $\begin{array}{l}\text { DMT1 [42] } \\
\text { Transferrin Receptor } 1 \text { (TFR1) [45] } \\
\text { Slc39a14 (ZIP14) [77] } \\
\text { Lactoferrin receptor (LfR) [43] } \\
\text { CD163 [45] } \\
\text { CD64 [220] } \\
\text { Ferroportin (FPN) [50] } \\
\text { Lipocalin-2 (LCN-2) [63] } \\
\text { Nramp-1 [45] } \\
\text { Hepcidin [53] }\end{array}$ & $\begin{array}{l}\text { SLC39A (ZIP) } \\
\text { SIc39a2 [77] } \\
\text { SIc39a4 [77] } \\
\text { SIc39a14 [77] } \\
\text { SLC30A (ZnTs) } \\
\text { SIc30a1 [77] } \\
\text { Slc30a3 [77] } \\
\text { SIc30a5 [77] }\end{array}$ & $\begin{array}{l}\text { CTR1 [84] } \\
\text { ATP7A [84] }\end{array}$ \\
\hline Neutrophils & $\begin{array}{l}\text { Nramp-1 [63] } \\
\text { Lipocalin-2 [63] } \\
\text { Myeloperoxidase [113] } \\
\text { Ceruloplasmin [119] } \\
\text { Lactoferrin [110] }\end{array}$ & $\begin{array}{l}\text { Calprotectin } \\
\text { (S100A8/S100A9 heterodimer) [13] } \\
\text { Calgranulin C } \\
\text { (S100A12) [124] }\end{array}$ & $\begin{array}{l}\text { Calgranulin C } \\
\text { (S100A12) [124] } \\
\text { ATP7A [118] } \\
\text { ATP7B [118] } \\
\text { Ceruloplasmin [119] }\end{array}$ \\
\hline NK cells & $\begin{array}{l}\text { TfR1 (CD71) [183] } \\
\text { Lactoferrin receptor (LfR) [185] }\end{array}$ & $\begin{array}{l}\text { KIR receptor [188] } \\
\text { Surface receptors with tyrosine phosphorylation } \\
\text { sites [190] }\end{array}$ & Unknown \\
\hline Dendritic cells & Unknown & ZIPs and ZNTs [102] & Unknown \\
\hline Basophils & Unknown & Methallothioneins (MTs) [158] & Unknown \\
\hline Eosinophils & Eosinophil peroxidase (EPX) [152] & Unknown & Unknown \\
\hline Mast cells & Unknown & Unknown & Ctr2 [161] \\
\hline y $\delta$ T cells & $\begin{array}{l}\text { Lactoferrin receptor (LfR) [195] } \\
\text { Hereditary hemochromatosis susceptibility } \\
\text { gene (HFE) [197, 198] } \\
\text { B2-microglobulin ( }(\mathrm{mm}-2)[198]\end{array}$ & Unknown & Unknown \\
\hline iNKT cells & $\begin{array}{l}\text { Ferroportin (FPN) [201] } \\
\text { Hepcidin [201] }\end{array}$ & Unknown & Unknown \\
\hline MAIT cells & Unknown & Unknown & Unknown \\
\hline T cells & $\begin{array}{l}\text { TfR1 (CD71) [209] } \\
\text { Lactoferrin receptor (LFR) [195] }\end{array}$ & Unknown & Unknown \\
\hline B cells & TfR1 (CD71) [209] & Unknown & Unknown \\
\hline
\end{tabular}

enhancing their bactericidal and phagocytic capabilities [59]. Importantly, erythrophagocytosis also upregulates the expression of heme oxygenase 1 (HO-1) in macrophages leading to heme degradation. Heme catabolism by HO-1 exerts anti-inflammatory effects through the products of heme degradation: bilirubin (arising from the reduction of biliverdin), carbon dioxide and ferritin all of which possess anti-inflammatory activity [60-62]. This illustrates the importance of iron metabolism, particularly of the HO-1/heme axis in maintaining AM function and lung homeostasis.

Iron-sequestration is of particular importance to limit pathogen outgrowth in the lungs. AMs act as the first line of defence against respiratory pathogens and contribute to limiting iron availability to pathogens. An example is the ability of AMs to secrete the siderophore lipocalin-2 (LCN-2) during infection that binds to enterobactin-type and mycobacterial siderophores to sequester free iron (Fig. 2) [63]. Macrophage polarisation also differentially regulates iron-response in AMs. Iron accumulation in M1-polarized AMs promotes a bacteriostatic response to the anaemia of chronic infection and simulates expression of pro-inflammatory cytokines, potentially through hepcidin-mediated FPN downregulation [64]. In contrast, M2 cells favour iron release linked to upregulation of FPN a phenotype that may be driven by upregulation of HO-1 [65]. Reciprocally, iron can also modulate monocyte polarization. In vivo murine models of iron overload drive macrophage polarization to favour the M2 phenotype mitigating pro-inflammatory responses [66]. However, in vitro studies of macrophages isolated from chronic venous leg ulcers showed excessive erythrophagocytosis and an ensuing high intracellular iron concentration to promote M1 polarization [67]. Likely, iron concentration is crucial in modulating polarisation the tissue iron deposition exhibited in venous leg ulcers is extremely high, whilst the dietary iron supplementation approach used in the murine studies provides a moderate dose [66]. While the effects of iron overload in the lung on macrophage polarization remain unclear, it will be interesting to see if such a concentration-dependent mechanism is also observed. 


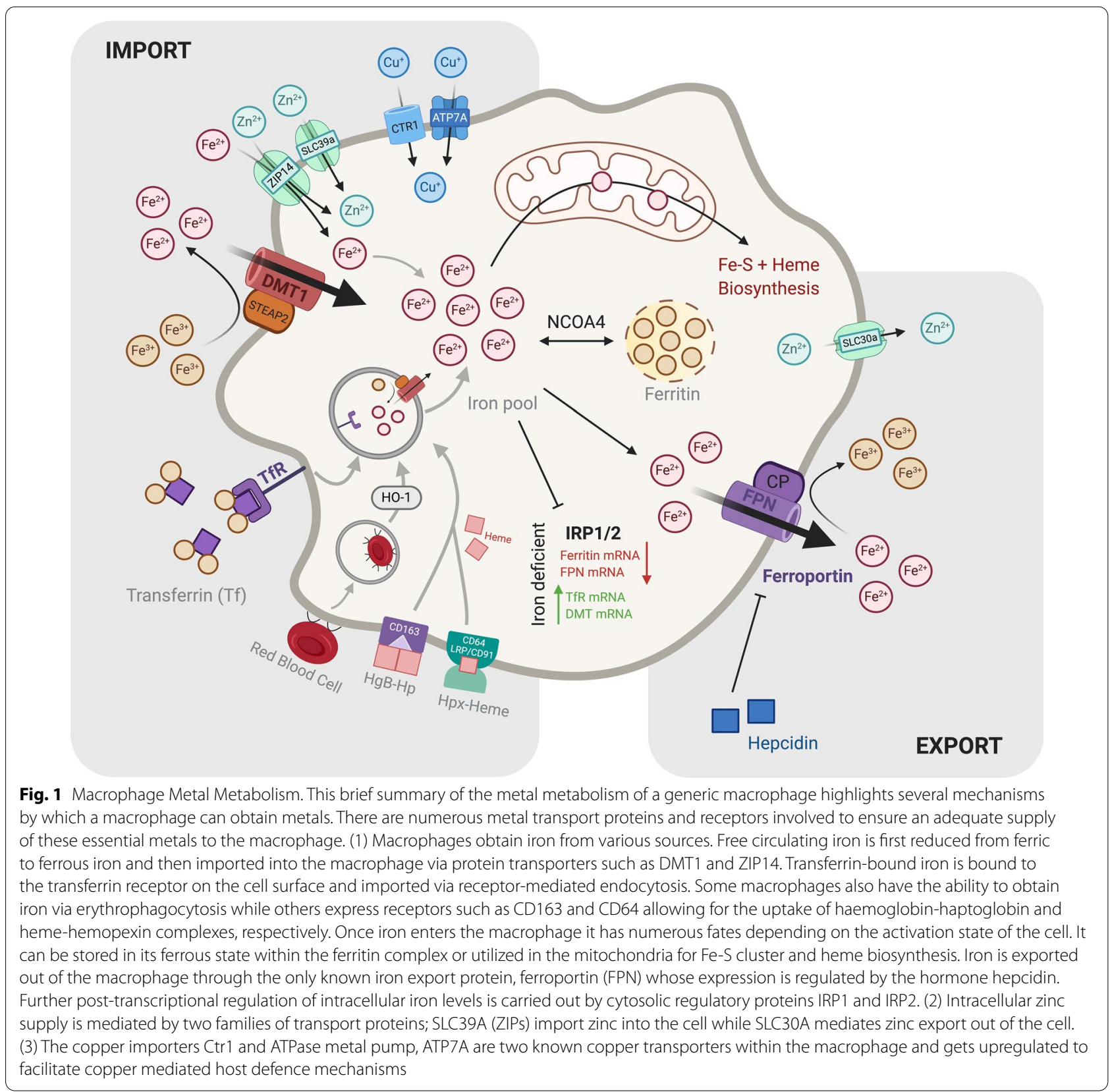

Macrophages carry out a vast array of effector functions and must be able to adopt several activation states by changing their metabolism to fuel these functions [68]. The influence of macrophage immunometabolism in determining an inflammatory or anti-inflammatory phenotype have been extensively reviewed [69]. However little attention has been given to the role that metals play in the regulation of immunometabolic networks. Iron-sulfur (Fe-S) clusters play an essential role in macrophage metabolism by acting as cofactors to essential metabolic proteins and mediating the electron transport chain. For example, the mitochondrial enzyme lipoic acid synthase (LIAS) requires Fe-S clusters to aid in the synthesis of lipoamide-requiring enzymes such as pyruvate dehydrogenase $(\mathrm{PDH})$ and $\alpha$-ketoglutarate dehydrogenase $(\alpha-K G D H)$. Furthermore, the proteins involved in electron transport, such as ferredoxins also require Fe-S clusters as a part of their redox-active centres to ultimately produce ATP [70]. In response to inflammatory stimuli Fe-S clusters are also regulated. LPS/interferon- $y$ (IFN- $\gamma)$ stimulation of murine bone marrow derived macrophages (BMDMs) and RAW264.7 macrophages 


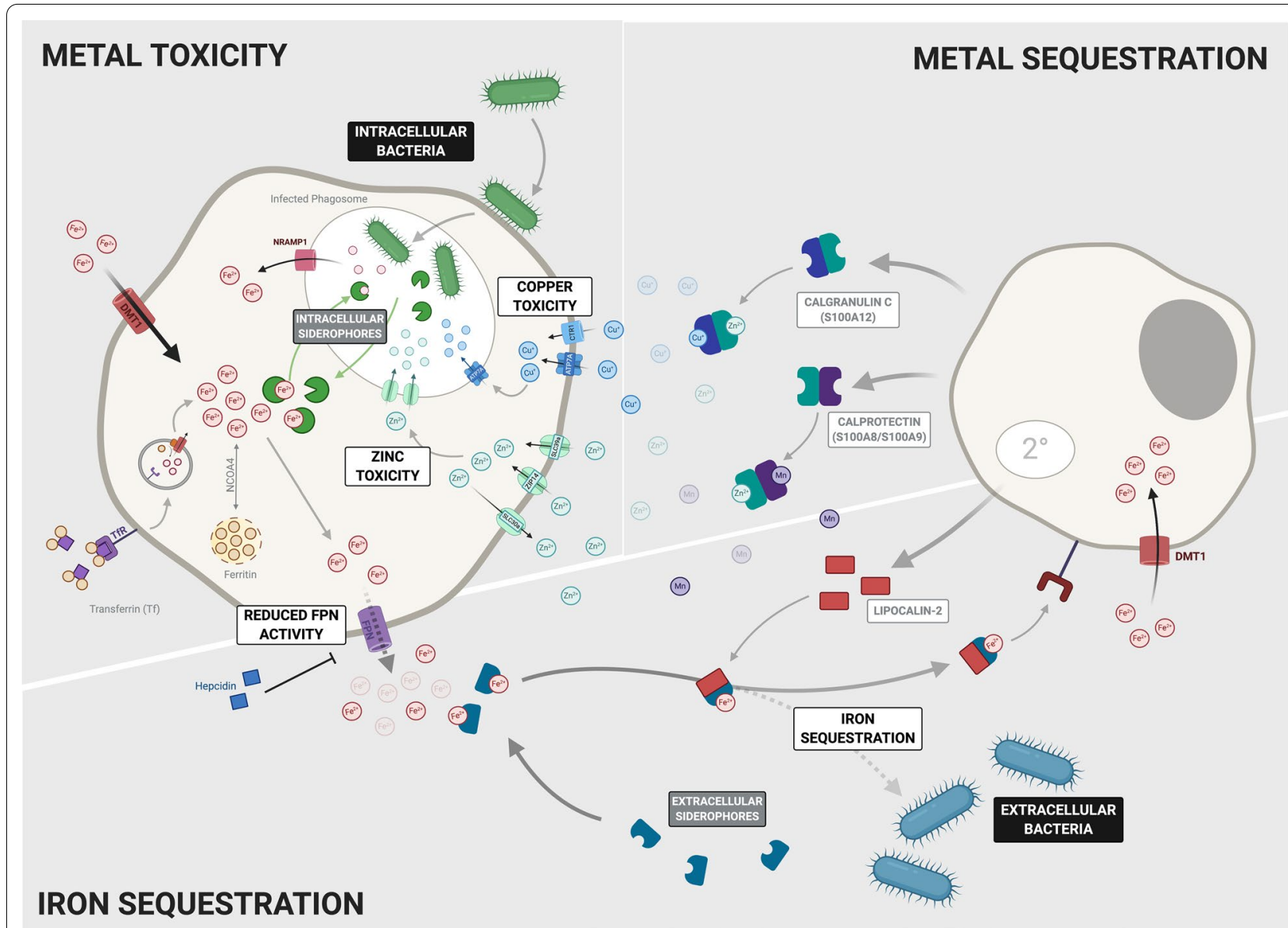

Fig. 2 Host Bactericidal Immune Defence Facilitated by Trace Metals. Metals play a pivotal role in contributing to the host immune response during bacterial infection. They can be used by immune cells to either mediate metal toxicity to rapidly kill the bacteria or be sequestered away in order to deprive the bacteria of essential metals in order to limit bacterial growth and replication. Whether the metals are sequestered or used to facilitate toxicity mechanisms is highly dependent on the nature of the bacterial infection. (1) Intracellular bacteria are mainly located within the phagosome of their host cell. Here they release siderophores allowing them to scavenge iron within the cell. In response, the host limits bacterial iron supply by exporting iron out of the infected phagosome via the NRAMP1 transporter. Furthermore, the host employs toxicity mechanisms by which both zinc and copper are pumped into the infected phagosome via their respective transporters in order to limit bacterial growth and replication. (2) Extracellular bacteria produce numerous different proteins to mediate metal acquisition from the host. The majority produce siderophores, which vary between bacterial species. However, they all function to supply the bacteria with an adequate amount of iron. The host also employs mechanisms to limit the amount of metals available to the bacteria in the extracellular space. Iron is sequestered by the upregulation of hepcidin resulting in the degradation of FPN which reduces the amount of iron exported from the cell. Furthermore, the iron importer DMT1 is also upregulated in both macrophages and neutrophils. Several metal binding proteins have also been shown to be produced mainly by neutrophils to bind extracellular metals. Lipocalin2, produced in secondary granules of neutrophils, binds bacterial siderophores and shuttles them away from the bacteria to the host cell. S100 proteins such as Calprotectin that binds zinc and manganese, and Calgranulin C binding zinc and copper also sequester these metals out of the extracellular space

downregulates two key Fe-S cluster biosynthesis proteins (cysteine desulfurase $\mathrm{Nfs} 1$ and its partner IscU) possibly to avoid damage to Fe-S clusters by NO [71]. In vivo stimulation with Mycobacterium bovis Bacille Calmette Guerin (BCG) or in vitro stimulation with IFN- $\gamma$ inhibits aconitase activity in macrophages by removing an iron atom for the Fe-S prosthetic group of aconitase which is essential for its catalytic activity in the TCA cycle. This inhibition of aconitase coincides with the downregulation of mitochondrial respiration upon its activation and also a compensatory increase of glycolysis [72]. Similarly, acute iron deprivation of primary human macrophages enhances glycolysis by upregulating hypoxia-inducible factor 1- $\alpha$ (HIF-1 $\alpha)$ regulated genes while downregulating oxidative phosphorylation (OX PHOS) via the ironresponsive transcription factor ATF-4. Furthermore, when these iron-deprived cells were stimulated with LPS their proinflammatory functions were impaired [73]. 


\section{Macrophages, zinc and copper}

Like iron, zinc is an essential cofactor for life. Vertebrates have evolved complex transport and buffering systems to maintain zinc homeostasis. The balance of zinc homeostasis is crucial during infection to block access to zinc from invading microorganisms, but also to ensure the function of immune cells. Zinc deficiency in humans is associated with impaired response to several infectious diseases [74, 75]. Zinc deficiency leads to reduced phagocytic ability of macrophages, while zinc supplementation can improve phagocytosis but the molecular mechanisms behind how zinc affects this process is not yet known [74-76]. Cellular zinc homeostasis is regulated by two families of zinc transporters, the SLC39A importers (ZIPs) and the SLC30A exporters (ZnTs). Zinc plays an important role in macrophage efferocytosis involving the coordinated action of the ZIP1 and ZIP2 importers [77]. The roles of other zinc transporters in different infection contexts is not yet known but the exporter SLC39A10 is vital for mediating zinc homeostasis in macrophages in response to LPS stimulation resulting in increased macrophage survival (Fig. 2) [78]. Slc30a1 (ZnT1) is induced in human macrophages during $M$. tuberculosis infection and zinc toxicity is a strategy used by $M$. tuberculosis infected macrophages [79]. Zinc accumulation was also observed in phagosomes containing $E$. coli contributing to its killing, suggesting that this may be a general antimicrobial strategy employed by macrophages [80].

Copper is used as an essential cofactor in an array of biological processes in many forms of life, including microorganisms. Copper deficiency increases susceptibility to many types of infections indicating an important role for this metal in host defence [10, 81, 82]. Furthermore, the prevalence of copper resistance mechanisms in pathogenic microbes highlights the importance of copper toxicity as a defence strategy in mammals. Macrophages exploit both the essentiality and toxicity of copper to defend against microbes. As a host defence strategy, copper levels rise within phagosomes of peritoneal macrophages upon infection with mycobacteria [83]. The copper importer Ctr1 and the ATPase copper pump ATP7A are induced by IFN- $y$ in macrophages. The ATPase pump is trafficked to phagosome compartments and is required for the bactericidal activity of macrophages (Fig. 2) [84]. Copper toxicity is a host defence strategy against other respiratory pathogens such as $S$. pneumoniae as pulmonary macrophages were shown to be more efficient at clearing pneumococcal bacteria lacking the CopA copper efflux pump [85]. Conversely, IFN- $\gamma$ activated macrophages reduce copper levels in phagosomes to control the fungal pathogen Histoplamsa capsulatum [86]. Fungi have high tolerance to copper and so copper toxicity as a defence strategy is not suitable for these micro-organisms, thus macrophages restrict this essential nutrient from fungi within the phagosome.

\section{Macrophages and other trace metals}

The role of other trace metals in macrophage biology is poorly studied and has mainly focused deciphering additional metal binding capabilities of proteins implicated in iron biology. In addition to iron, the membrane transporter NRAMP1 also has affinity for manganese, transporting it across the phagosomal membrane [87]. Transferrin has been shown to bind manganese in vitro and is the main manganese-positive protein in circulation in vitro, although whether macrophages can take up manganese-laden transferrin through TFR1 remains to be defined [88].

While numerous selenoproteins key in cellular processes have been characterised, their precise roles in processes governing the macrophage immune response are poorly defined. Selenoprotein methionine sulfoxide reductase B1 (MsrB1) expression is induced in macrophages upon LPS stimulation, where it regulates actin assembly and production of anti-inflammatory cytokines [89]. This anti-inflammatory role is concordant with observations that dietary selenium supplementation drives macrophages towards the alternatively activated M2 phenotype in an IL-4 dependent manner [90]. Macrophages deficient in the selenocysteine tRNA showed diminished migration in vitro, but no change in inflammatory response [91]. Targeting of specific selenoproteins in future studies will allow a greater understanding of the intricacies of selenium regulation in macrophages.

\section{Dendritic cells}

Dendritic cells (DCs) play a crucial role in the initiation and regulation of the immune response by functioning as powerful antigen presenting cells. DCs resident in the lungs can be divided into three subsets that are of independent origin and have distinct functions depending on their location [92]. Regardless of their subtype, DCs develop in the bone marrow from hematopoietic stem cells into early progenitor cells where they then branch off into their specific subsets [93]. Conventional DCs (cDCs) in the lung express either CD103 or CD11b. CD103 ${ }^{+}$DCs $(\mathrm{cDC} 1)$ are located in the respiratory epithelium where they sample contents of the conducting airways by projecting their long dendrites between epithelial cells into the airway lumen. In contrast, CD11b ${ }^{+}$DCs (cDC2) are lodged under the basement membrane of the lamina propria [94]. Once the cDCs sample an antigen, they migrate the draining lymph nodes where they present the processed antigen to naïve $\mathrm{T}$ cells which drives specific $\mathrm{T}$ cell polarization and proliferation [95]. 
Monocyte-derived DCs (moDC) are recruited to the lung during infection or inflammation to help drive the immune response. The existence of these DCs remains controversial as they express markers such as CD11c, CD64 and the MAR-1 antibody which can also represent a macrophage cell population [94, 96]. Plasmacytoid DCs (pDC) secrete large amounts of type I interferon (IFN) during viral infections and have been located in the lymphoid follicles of the small airways [97, 98]. Furthermore, pDCs also play an immunoregulatory role by sensitising $\mathrm{T}$ helper (Th) type-2 cells to harmless antigens in the lung while suppressing the generation of effector $\mathrm{T}$ cells. However, this tolerance is suggested to be suppressed when the pDCs interact with a virus and produce IFNs [99].

\section{Dendritic cells and metals}

Not much is known about the role that metals may play in the function of dendritic cells. Iron levels affect the development of DCs from bone marrow progenitors whereby high iron conditions used to culture bone marrow cells leads to a defective development of moDCs that are unable to respond efficiently to LPS [100]. On the other hand, iron deficiency in vitro also leads to the improperly differentiated moDCs that are unable to stimulate $\mathrm{T}$ cells [101]. Interestingly, reduced intracellular free iron levels promote the activation of DCs. Stimulation of DCs with LPS leads to an NFKB dependent increase of ferritin.

Zinc has also been implicated in DCs response to stimulation. A study identified a link between toll like receptor (TLR) signalling and zinc homeostasis in these immune cells. Stimulation of DCs with the TLR4 ligand LPS lead to altered expression of zinc transporters (both importers and exporters), and a decrease in intracellular free zinc [102]. Treating DCs with a zinc chelator resulted in the same effects as LPS stimulation, in terms of upregulation of MHC class II and costimulatory molecules. Further investigations are needed to understand the link between DC zinc homeostasis and their response to TLR stimulation.

\section{Neutrophils}

Neutrophils are short-lived, highly mobile phagocytes that constitute hallmarks of acute infection. As granulocytes neutrophils have enzyme-filled granules that they use to fight off infections in addition to their phagocytic capabilities and synthesis of chromatin-derived neutrophil extracellular traps (NETs) [103]. Despite their prominent phagocytic role in the response to acute infection, neutrophil function is remarkably plastic, with secreted effectors driving pro- and anti-inflammatory neutrophil function [104-106]. Neutrophils also possess the capacity to modulate the functionality of other immune cells through secreted effector proteins, with the cleavage of TLRs and cytokines by neutrophil elastase associated with altered macrophage function [107]. Given its frequent exposure to environmental pathogens, it is no surprise that the lung is a major neutrophil reservoir. Studies in a rabbit model have shown the upregulation of P-selectin by AECs in response to internal and external stimuli binds P-selectin glycoprotein ligand-1 on circulating neutrophils, facilitating further binding and subsequently tissue extravasation [108]. The majority of marginated neutrophils in the lung reside in the capillary bed, expressing the chemokine receptor (CXCR) 4 to promote their retention [109]. Such margination in the microvasculature positions a large pool to kill inhaled bacterial, fungal and viral pathogens through phagocytosis, NETosis and release of antimicrobial cytokines [107].

\section{Neutrophils and iron}

Iron is central to numerous neutrophil inflammatory responses. Neutrophils secrete LCN2 from their secondary granules to bind bacterial siderophores and limit their iron acquisition, and express NRAMP1 to sequester free iron from serum during infection [63]. Studies in the iron transport protein lactotransferrin (lactoferrin)-deficient mice have illustrated a role of neutrophil-secreted lactoferrin in modulating the oxidative burst response [110]. Iron-unsaturated lactoferrin was shown to inhibit human neutrophil apoptosis in vitro, suggesting a role in chronic neutrophilic inflammation [111]. Binding of recombinant human lactoferrin to human neutrophils in vitro induces neutrophil activation although the precise mechanisms of lactoferrin binding to the neutrophil surface remain uncharacterised [112]. Neutrophils also express the heme-containing myeloperoxidase, which catalyses the generation of hypochlorite anions to drive degranulation and NETosis [113].

Iron availability may also influence metabolic reprogramming in neutrophils. HIF- $1 \alpha$ is regulated by a family of prolyl hydroxylases (PHDs) and under normoxic conditions the PHDs rapidly hydroxylate HIF- $1 \alpha$ and so target it for degradation [114]. Phd $2^{-/-}$neutrophils have higher ATP levels, increased abundance of pentose phosphate pathway intermediates, more glycogen stores and a greater glycolytic capacity [115]. This suggests that neutrophils, like macrophages, require HIF- $1 \alpha$ to engage in glycolysis. Low oxygen tension is not the only way to block HIF- $1 \alpha$ hydroxylation, as PHDs also require $\alpha$-ketoglutarate, $\mathrm{Fe}^{2+}$ and ascorbate. Increased expression of HIF- $2 \alpha$, regulated in a similar manner to HIF- $1 \alpha$, has been linked to decreased death of neutrophils by apoptosis, and iron chelators have been shown to inhibit apoptosis in neutrophils [116]. Heme metabolism has also been implicated in neutrophil function during respiratory infections as $\mathrm{HO}-1$ expression in the lung is 
also associated with reduced neutrophil recruitment in murine models of LPS-induced pulmonary inflammation [117].

\section{Neutrophils and other metals}

Copper is required for proper NETosis, with mice carrying mutations in the copper transporters ATP7A and ATP7B showing a reduced capacity for NET release during systemic inflammation [118]. Ceruloplasmin, a $\mathrm{Cu}^{2+}$ dependent ferroxidase elevated in serum during acute infection, is secreted by human neutrophils to upregulate ROS in a potentially bactericidal mechanism [119]. Calprotectin is released from the cell during degranulation as part of NETs, where it is essential for antifungal activity [13].

Neutrophil expression of protein effectors binding other trace metals is crucial in their bactericidal function. Zinc deficiency induces expression of the zinc-binding heterodimer calprotectin and detrimentally impacts neutrophil phagocytic capacity through impairing ROS function, along with oxidative burst, degranulation and cytokine production [120]. Indeed, zinc signalling has been shown to be vital in NET formation by neutrophils and granulocytes: both zinc excess and deficiency inhibit superoxide production in neutrophils, a prerequisite for NET formation [121]. Neutrophils express calprotectin which can bind zinc and manganese, attenuating the effects of zinc-associated enzymes such as matrix metalloproteases and manganese-dependent bacterial superoxide defences [122, 123]. Neutrophils also express calgranulin C (S100A12), which can bind copper and zinc to exert an antimicrobial effect and trigger monocyte recruitment [124]. In addition to iron and zinc, calprotectin can also chelate manganese in a manner that both sequesters the nutrient from pathogens and facilitates antimicrobial activity, with recombinant calprotectin defective in manganese binding unable to exert antifungal activity against Aspergillus fumigatus [125]. Indeed, calprotectin-mediated manganese and zinc sequestration is a host immune strategy against the $S$. aureus superoxide defence, enhancing the susceptibility of the bacterium to neutrophil killing [122] Little is known about the role of selenium in neutrophil function, although increased dietary intake may protect neutrophils from oxidative damage [126].

\section{Other granulocytes \\ Mast cells}

Mast cells are granulocytic leukocytes that mediate inflammation through degranulation in response to infection, allergens and tissue injury [127]. Mast cells are unique among leukocytes in that they are released from the bone marrow as $\mathrm{CD} 34^{+}$mast cell progenitors and do not terminally differentiate until their recruitment to tissue [128]. These immature progenitors are minimally granulated and reach maturation upon tissue homing, where stem cell factor and other tissuespecific factors drive MCs towards a pro-inflammatory, anti-inflammatory or an immunosuppressive phenotype [129]. Mast cell activation is regulated by several cell surface receptors, including TLRs, cytokine and chemokine receptors, hormone receptors and FceRI and Fc $\gamma$ receptors. The ensuing combination of stimuli facilitates a pleitropic role for mast cells in the immune response and leads to the secretion of a wide range of effector proteins: preformed mediators rapidly released upon activation (TNF- $\alpha$, histamine and proteoglycans), and mediators synthesised following activation (lipid mediators, growth factors, cytokines and chemokines) [130-132]. Mast cell frequency in the lung at homeostasis is low and interspecies variation in mast cell populations have made the homing process difficult to study, although the identification of a multitude of mast cell chemoattractants means it likely occurs through a regulated recruitment process $[133,134]$.

\section{Basophils}

Basophils similar to mast cells contain granules within their cytoplasm [135], express the high affinity receptor for IgE (FceRI) and release proinflammatory mediators such as histamine when this receptor becomes cross-linked. Both are derived from $\mathrm{CD}_{3}{ }^{+}$haematopoietic stem cells located in the bone marrow [136]. Despite their similarities, basophils and mast cells are morphologically and biochemically distinct cell types [137]. The type 2 immune response is initiated by the immune system when in contact with parasites which increases IgE production and the number of eosinophils, basophils and CD4 T cells [138]. Basophils drive this type 2 response by producing the cytokine IL-4 [139] but also plays a role in allergic reaction and airway inflammation. Basophils have quite a short lifespan and so they must be constantly replenished by the bone marrow. For many years it was accepted that basophils are present in circulation and only migrate to tissue in response to certain inflammatory signals [140]. However, murine studies have proposed the idea of tissueresident basophils after it was discovered that basophils seem to be present at all stages of lung development [141]. Furthermore, these lung-resident basophils differ phenotypically from peripheral blood basophils and are driven by IL-33 and GM-CSF produced in the lung microenvironment. Localised in the alveoli, intriguingly basophils also seem to be responsible for guiding the development and function of AMs [141]. 


\section{Eosinophils}

Eosinophils are bone marrow-derived, granule containing, pro-inflammatory leukocytes. Terminally differentiated in circulation, eosinophils act as effectors in host defence from microbial and metazoan infection and allergic reaction, primarily through the activity of cationic proteins resident in the eosinophilic granule. Eosinophils comprise a mere $1-3 \%$ of circulating leukocytes and readily migrate to the lung during inflammation. Following their IL-5-induced proliferation and mobilization in the bone marrow, eosinophil recruitment into the lung is driven by local expression of the chemotactic factor eotaxin [142]. Lung-resident eosinophils reside in the parenchyma and display a marker expression pattern (Siglec- $\mathrm{F}^{\text {mid }} \mathrm{CD} 62 \mathrm{~L}^{+} \mathrm{CD} 101^{\text {low }}$ ) distinct from those recruited to the airways (Siglec- $\mathrm{F}^{\text {hi }} \mathrm{CD} 62 \mathrm{~L}^{-} \mathrm{CD} 101^{\text {hi }}$ ) although functional differences between the two subsets remain uncharacterised [143]. Murine models of chronic allergic asthma have shown secretion of CCL17 and CCL22 by dendritic cells recruits circulating eosinophils to the lung in response to allergic inflammation, where eosinophils recruit further DCs and Th2 cells [144, 145]. During viral respiratory infection, expression of TLR7 allows eosinophils to detect pathogen associated molecular patterns (PAMPs) and exert direct antiviral effects through degranulation and cytokine and superoxide production [146].

\section{Metals and granulocyte function}

There are many reports correlating altered iron metabolism with allergy and atopic airway hyperreactivity. There is a higher prevalence of allergic diseases in those with diseases causing high systemic iron levels [147]. As basophils, eosinophils and mast cells play a role in allergy, the altered iron homeostasis likely impacts the function of these cells, contributing to pathology. Reducing iron levels, by administration of an iron chelator reduces $\operatorname{IgE}$ serum levels in a rat model of Th2 mediated autoimmunity [148]. Iron supplementation results in a significant decrease of airway eosinophila in an ovalbumin-driven allergic asthma mouse model [149]. Neutrophils utilize the metalloprotein myeloperoxidase (MPO), which is a hemeprotein that plays a role host defence via oxidation [150]. Similarly, eosinophils have eosinophil peroxidase (EPX), which is a two-chain hemeprotein and has been shown to have homology to MPO in neutrophils [151]. Efficient iron stores and uptake is said to be required for the generation of EPX suggesting iron levels are important for eosinophil function and activity [152].

HIF- $1 \alpha$, whose activation is iron dependent was shown to accumulate after basophils were stimulated with anti-IgE [153]. Accumulation of HIF- $1 \alpha$ is suggested to be linked to the reaction of basophils to the hypoxic environment and stress-induced conditions. Whether intracellular iron levels in basophils affects HIF1 $\alpha$ and cell activation as it does in macrophages and dendritic cells remains to be determined. This would be an interesting avenue to explore as altered iron levels are linked with allergic inflammation.

Reduced zinc levels are associated with several allergic diseases including bronchial asthma and chronic rhinosinusitis [154-156]. Zinc is required in mast cells for degranulation and the production of cytokines and mast cell granules are reported to contain high levels of zinc [157]. FceRI is expressed on the surface of mast cells and basophils, and its activation leads to the release of cytokines (including IL-4) and chemical mediators. The requirement of zinc in basophil function has not yet been fully elucidated however the regulation of intracellular zinc levels by metallothioneins (MTs) has an important role in FceRI-driven calcineurin/nuclear factor of $\mathrm{T}$ cell signalling and IL-4 signalling in basophils [158]. Zinc suppresses eosinophilic inflammation, increased numbers of eosinophils in bronchoalveolar lavage fluid fluid (BALF) are observed in zinc deficiency while zinc supplementation reduces the numbers of eosinophils in BALF, similar to what is observed with iron $[159,160]$. However, the molecular mechanism driving this zincassociated eosinophil recruitment to the lungs is yet to be elucidated. While copper has been implicated in mast cell maturation, little remains known about the role of copper and other metals in granulocyte function [161].

\section{Innate lymphocytes}

In addition to the innate and adaptive defences, the lung harbours innate-like lymphocytes types that constitute the second and third tiers of defence. Lung tissue-resident innate-lymphocytes include innate lymphocyte cells (ILCs) which include NK cells, invariant natural killer cells (iNKT), MR1-restricted T (MAIT) cells and $\gamma \delta \mathrm{T}$ cells.

Natural killer (NK) cells are short-lived innate effectors that belong to type 1 innate lymphoid cells, and account for $5-25 \%$ of total $\mathrm{CD} 45^{+} \mathrm{Lin}^{-}$cells in the lungs [162, 163]. NK cell activation is mediated through expression of inhibitory and activating receptors. Inhibitory receptors (killer cell-immunoglobulin like receptor (KIR), CD94:NKG2A, ILT-2) recognise host major histocompatibility complex class I molecules to limit cytotoxicity towards the host and facilitate self-tolerance in the 'missing self' response [164]. Activating receptors (TLRs, NGK2D) recognise increased ligand expression on target cells to mediate the 'induced self' response [165]. NK cells migrate from the bone marrow to the lung. In humans, NK cells are found in the lung parenchyma whereas in mice they have been found in the alveoli during influenza 
infection $[166,167]$. In the human lung NK cells constitute several heterogenous populations. CD $16^{+} \mathrm{CD} 56^{\mathrm{dim}}$ cells constitute $80 \%$ of lung NK cells and are a hyporesponsive, mature, cytotoxic population that express KIR [168]. Whether the lung contains a tissue resident population of NK cells has been debated. Marquardt et al. showed lung NK cells lack expression of the CD69 tissue resident marker; however, Yamamoto et al. showed that blocking NK cell recruitment has no effect on NK ability to control lung tumour growth, suggesting lung-resident NK cells are essential in controlling metastasis $[167,169]$. Parabiotic mouse studies have shown this lung-resident population to be low relative to other tissues with a specific tissue and functional signal, potentially contributing to barrier functions in the lung [170]. Murine lung NK cells also have diminished proliferative capacity and cytotoxic activity than splenic NK cells [171], suggesting that the lung microenvironment may inhibit NK cytotoxic potential to prevent airway damage. NK cells play important roles in response to infection and cancer. Murine models have illustrated their importance against respiratory viruses, bacteria and fungi [172]. Their precise contribution during human lung infections is unclear, but they are important in the early response to influenza [173]. Loss-of-function mutations leading to NK cell deficiencies uncovered an increased susceptibility to recurrent bacterial respiratory infections [174]. NK cells have also been implicated in asthma [172] and COPD [172].

$y \delta \mathrm{T}$ cells are a subset of unconventional or innate-like $\mathrm{T}$ cells that are characterized by the expression of a $\gamma$ and $\delta$ chain $\mathrm{T}$ cell receptor (TCR) which require MHC for antigen recognition. $\gamma \delta \mathrm{T}$ cells are abundant in mucosal surfaces including the lungs [175] where they swiftly react to conserved non-peptide antigens and produce large amounts of cytokines. In the lungs, $\gamma \delta \mathrm{T}$ can constitute up to $20 \%$ of resident lymphocytes and contribute to maintain tissue homeostasis and fight infections and cancer. These cells play important roles against bacterial pathogens including S. pneumoniae, B. pertussis, $M$. tuberculosis, viruses i.e., influenza, and some fungi. Likewise, they promote protection in models of cancer. However, these cells can also contribute to pathology in asthma and lung fibrosis [176].

In addition to NK cells, ILCs include a heterogeneous population of other innate lymphocytes described both in mice and humans. These include lymphoid tissue inducer (LTi) cells and three subsets of 'helper' ILCs (ILC1, ILC2 and ILC3) which unlike NK cells, lack cytotoxic properties and secrete higher amounts of effector cytokines. Lung helper ILCs have been extensively reviewed [177]; these cells are present across all different parts of the respiratory tract and lungs, including upper respiratory tract, lung parenchyma and bronchoalveolar spaces, where they contribute to homeostasis, pathogen clearance and also pathology [177].

Invariant natural killer cells (iNKT) and MR1-restricted $\mathrm{T}$ (MAIT) cells are "innate-like" unconventional $\mathrm{T}$ cells that reside in the lung and other mucosal surfaces and play important roles during infection by recognizing non-peptidic antigens [178]. iNKT cells are $\alpha \beta$ T cells which recognize glycosphingolipids and other microbial-derived glycolipids presented in CD1d molecules. iNKT cells can swiftly produce a range of inflammatory cytokines when activated and type I iNKT also present cytolytic activity against cancerous and virally infected cells $[178,179]$. Lung type I NKT cells are mainly resident within the parenchyma (NKT17, which produce IL-17) or in the vasculature (NKT1 and NKT2) [180, 181]. MAIT cells recognize microbial-derived metabolites [178] and produce cytokines including IFN- $\gamma$, TNF- $\alpha$, IL-17A, and IL-22 aiding in bacterial killing [182]. Their role and precise localization in the lung in homeostasis remains to be determined.

\section{Metals and innate lymphocytes}

The influence of metals on innate lymphocytes has been less studied and seem to vary among different innate lymphocytes subsets.

\section{NK cells and Iron}

In contrast to monocytes and polymorphonuclear cells, little is known about a potential role for an iron-related nutritional immune response in NK cell function, although recent studies suggest a role for iron signalling pathways in NK cell activation. Specifically, expression of the transferrin receptor CD71 is upregulated in the NK cell surface during their maturation in the murine bone marrow and during activation with poly(I:C) [183]. Basal expression of CD71 in human NK cells at homeostasis is low and increases in response to cytokine treatment in vitro, although how this potential to increase iron uptake drives the activated NK cell phenotype is unclear [184]. Lactoferrin increases NK cell cytotoxicity in vitro, which could imply an antimicrobial role for NK cells in infection in response to neutrophil and macrophage secretion of lactoferrin [185]. NK cells of patients exhibiting systemic iron overload in myelodysplastic syndromes displayed upregulated c-Jun $\mathrm{N}$-terminal kinase (JNK) and downregulated p38 expression, suggesting a role for iron in NK signal transduction pathways [186]. It is interesting to note that perturbed iron homeostasis in NK target cells affects NK cell recognition-iron depletion and ferritin heavy chain (FTH) in primary cancer cells was observed to increase NK cell targeting [187]. 


\section{NK cells and other metals}

Zinc signalling has also been suggested as a mediator of the NK cell phenotype. Recognition of surface MHC-1 on endogenous cells by the NK cell KIR is essential in for NK cell-mediated killing. Zinc is essential for KIR multimerization to form this 'NK cell synapse' [188]. Zinc supplementation in vitro stimulates the differentiation of human-derived CD34 ${ }^{+}$progenitors to NK cells [189]. NK cells also possess several surface receptors with tyrosine phosphorylation sites, suggesting a role for zinc in signal transduction; the cytotoxic capabilities of NK cells are reduced in zinc deficiency and increased in zinc excess, with the precise signalling pathways driving such a change remaining unclear [190]. Roles for other trace metals such as copper or manganese in NK cell biology are difficult to decipher; the few studies attempting to ascertain this were largely performed in vitro and thus may not hold significant physiological relevance [191]. Manganese supplementation promotes NK cell antitumoral activity in vivo [192]. Manganese also activated the adhesion protein lymphocyte function-associated antigen (LFA)-1, essential for NK cell cytotoxicity [191]. In vitro supplementation with selenium increases splenic NK cell cytotoxicity [193]. Further in vivo studies are required to ascertain the exact role of manganese and selenium on NK cell function and the biological pathways implicated.

\section{iNKT, MAIT, $\gamma \delta T$ cells and metals}

Limited data on $\gamma \delta \mathrm{T}$ cells suggest that variations in iron availability may not affect these cells as much as they affect their adaptive lymphocyte counterparts. $\gamma \delta$ $\mathrm{T}$ cell express the TFR1 (or CD71) which is required by $\alpha \beta C D 4^{+}$and $\mathrm{CD} 8^{+} \mathrm{T}$ cells during proliferation. While blocking TFR1 with an anti-CD71 antibody blocks proliferation of T cells, $\gamma \delta \mathrm{T}$ cells proliferation is not affected, suggesting these cells are either less dependent on iron or rely on other mechanisms for obtaining iron [194]. Indeed, activated $\gamma \delta \mathrm{T}$ cells express high levels of the lactoferrin receptor (LfR). Like transferrin, lactoferrin can bind 2 ferric ions and provide LfR-expressing cells of an iron source; in addition lactoferrin possesses antimicrobial activity [195]. Unlike transferrin, which is present at high concentrations in plasma, lactoferrin is abundant in secretions like breast milk, and fluids covering the linings of mucosae like saliva, mucus and BALF $[195,196]$. Addition of lactoferrin to in vitro cultures increase the proliferation of $\gamma \delta \mathrm{T}$ cells upon mitogen stimulation. Given the important role of $\gamma \delta \mathrm{T}$ cells in defence against mucosal pathogens, high expression of LfR may be an adaptation for acquisition of lactoferrin-bound iron in mucosal sites including the lungs.
A functional link between the hereditary hemochromatosis $(\mathrm{HH})$ susceptibility gene HFE (also known as homeostatic iron regulator), iron status and adaptive $\mathrm{T}$ cell function has been suggested [197]. However, if or how HFE regulates $y \delta$ T cells remains unknown. HFE encodes a non-classical MHC molecule; as such the protein may have the ability to form complexes with $\beta 2$-microglobulin ( $\beta 2-\mathrm{m})$ [198]. Interestingly, mice deficient in $\beta 2-\mathrm{m}$ display iron overload patterns similar to $\mathrm{HH}[199]$ and have enhanced homeostatic proliferation of $\gamma \delta T$ cells. Since HFE deficiency is linked to iron overload, it is possible to hypothesize that excess iron could be linked to the altered $\gamma \delta \mathrm{T}$ cell proliferation in these mice and maybe also in $\mathrm{HH}$ patients. In addition to the altered lymphocyte ratios, some $\mathrm{HH}$ patients may have altered iNKT numbers. Iron overload is associated with reduced numbers of iNKT cells, which was more notable in untreated patients [200]. Intriguingly, the regulation of iron levels and iNKT activation/proliferation seems to be twoway: iron levels affect iNKT numbers, and iNKT cells can affect iron homeostasis. In vivo, activation of mouse iNKT cells by injection of their prototypical antigen $\alpha$-galactosylceramide ( $\alpha$-GalCer), not only induces iNKT proliferation but also promotes early hepcidin expression while suppressing FPN. Activation of iNKT cells also lead to an early peak of serum iron followed by accumulation of iron in the spleen and liver. These effects were abolished in $J \alpha 18^{-/-}$mice which particularly lack iNKT cells. This indicates that iron and iNKT cells reciprocally regulate each other [201]. More research is needed to understand the effects of iron and other metals on MAIT cells and other groups of innate lymphocytes.

\section{Metals and adaptive immune cells of the lung Adaptive lymphocytes ( $B$ and T cells)}

$\mathrm{T}$ and $\mathrm{B}$ cells are at the centre of cellular adaptive immune responses. These cells are virtually capable to recognize infinite antigens in a highly specific manner thanks to their TCR and B cell receptors (BCR) and offer long-term protection against infection thanks to the generation of effector and tissue resident memory $\mathrm{T}$ lymphocytes and long-lived antibody producing plasma cells. Adaptive lymphocytes can be found in different lungs compartments. Conventional $\mathrm{CD}^{+}{ }^{+}$and $\mathrm{CD} 8^{+} \mathrm{T}$ lymphocytes populate the mucociliary epithelium of the conducting airways in the trachea and bronchi. In contrast, $\mathrm{B}$ cells (mostly IgA ${ }^{+}$) and most $\mathrm{CD} 4^{+} \mathrm{T}$ cells are found in the bronchial lamina propria. Early studies also reported the presence of lymphocytes in the lung interstitium in similar numbers to those found in the circulation [202]. In addition, an intravascular pool has been identified. $T$ $\mathrm{CD}^{+}, \mathrm{T} \mathrm{CD}^{+}$and $\mathrm{B}$ lymphocytes have been isolated 
from the alveolar spaces. B cells constitute only $5-10 \%$ of the total lymphocytes isolated in BALF [202].

$\mathrm{T}$ and $\mathrm{B}$ lymphocytes can also accumulate in tertiary lymphoid structures (TLS) in the lungs. Bronchial Associated Lymphoid Tissue (iBALT) are the main TLS in the lung and serve as priming structures for $\mathrm{B}$ and $\mathrm{T}$ cells. iBALT organization is similar to secondary lymphoid organs, presenting a $\mathrm{T}$ cell zone and a $\mathrm{B}$ cell follicle. These structures are readily detectable in children but only inducible and transient in adults. Once iBALT is formed, it can be maintained in the lungs for months even in the absence of the original stimulus that triggered it and serves as a transient lymphoid structure to recruit naïve lymphocytes from the blood and to promote their interaction with local antigens in the airways to favour activation and differentiation into effector cells. The iBALT is formed near the basal side of the bronchial epithelium and in close association with the pulmonary blood vessels in response to infection or other inflammatory stimuli [203]. iBALT formation is protective against acute viral and bacterial respiratory infections and it has been also shown to contribute during chronic $M$. tuberculosis infection. However, activation of lymphocytes in TLS can also contribute to airway damage. For instance, these structures tend to form near small airways in COPD patients and are associated with more severe forms of the disease [204].

Lung resident memory $\mathrm{T}$ cells $\left(\mathrm{T}_{\mathrm{RM}}\right)$ protect against infection by expanding rapidly upon challenge. $\mathrm{CD} 4^{+}$ $T_{R M}$ reside in the lung parenchyma, whereas $C D 8^{+} T_{R M}$ are found in parenchyma and the airway epithelium. $\mathrm{CD} 4^{+} \mathrm{T}_{\mathrm{RM}}$ are maintained over longer periods of time while $\mathrm{CD} 8^{+} \mathrm{T}_{\mathrm{RM}}$ decay and must be replenished from circulating $\mathrm{T}$ effector memory cells [205]. A subset of memory $\mathrm{CD} 69^{+} \mathrm{CD} 103^{+} \mathrm{T}$ regulatory (Tregs) cells expressing FoxP3 are also found in the lungs of mice and humans [206, 207]. Antigen-specific memory Tregs differentiate in response to certain respiratory infections such as influenza and can persist in the lungs after the pathogen has been cleared. These cells can contribute to limit the damage upon reencounter with the pathogen [207] and can limit the pro-fibrotic potential of $\mathrm{CD} 44^{\text {hi }} \mathrm{CD} 69^{+} \mathrm{CD} 103^{\text {lo }}$ $\mathrm{CD}^{+} \mathrm{T}$ cells [208].

\section{Metals and adaptive immune cells}

Trace metals including iron and zinc, as well selenium can influence adaptive lymphocyte biology and function. As for most immune cells, iron is key to T and B lymphocytes. Both express TFR1 or CD71 which allows them to acquire transferrin- $\mathrm{Fe}^{3+}$ via endocytosis. The acquired iron is used as a cofactor in several enzymes and a small proportion is stored in ferritin or remains as part of the labile iron pool in the cell; although the iron reserve pool in lymphocytes is very limited compared to other immune cells [209]. In addition to TFR1, lymphocytes also express LfRs upon activation which are particularly abundant on $y \delta$ T cells [195].

Both iron overload and iron deficiency impact adaptive immunity and lymphocyte function but the different $\mathrm{T}$ helper and cytotoxic lymphocyte subsets display different responses to iron perturbations and their dependence on TFR1 iron uptake. Mice fed an iron-rich diet show lower IFN-y production and impaired delayed-type hypersensitivity responses, whereas iron-deficient diets are linked to impaired T-cell proliferation [209]. In vitro, blocking TFR1 with antibodies causes the arrest of helper $\mathrm{T}$ cell (Th)1 cell proliferation, whereas Th2 cells do not seem to be affected. In contrast, supplementation of culture media with iron and transferrin boosts lymphocyte proliferation induced by polyclonal activators such as concanavalin A, phytohemagglutinin or LPS [210]. Supporting the importance of transferrin and iron for lymphocytes in the lungs, transferrin levels in the BALF of patients with COPD and sarcoidosis correlate with the number of lymphocytes in the BALF [211].

Iron levels are key to adaptive responses to pathogens and vaccines; anemia, iron or increased hepcidin and low serum iron caused by a mutation in TMPRSS6 predict reduced responses to rubella, diphtheria, pertussis, $H$. influenzae type B and pneumococcal vaccines $[212,213]$. Importantly, in humans another homozygous mutation in TFRC (the gene encoding TFR1) results in defective TFR1 iron internalization. Homozygous carriers of this mutation display a combined immunodeficiency syndrome that results from impaired $\mathrm{T}$ and $\mathrm{B}$ cell proliferation, defective class-switching and lower antibody production, demonstrating the importance of iron uptake and TFR1 for adaptive response and lymphocyte's function [214].

$\mathrm{T}$ cells are also affected by iron overload as seen in certain cohorts of patients with $\mathrm{HH}$ or transfusiondependent thalassemia. A subgroup of thalassemia patients present with unusually low counts of $\mathrm{CD} 8^{+}$ $\mathrm{T}$ cells, which can improve after subcutaneous chelation therapy with the iron chelator deferoxamine (DFO) [215]. A similar phenotype has been observed in $\mathrm{HH}$ patients. HFE competes with transferrin for binding to TFR1 and downregulating iron cellular uptake. Iron-sensing via the HFE-TFR1 axis ultimately induces hepcidin transcription blocking iron efflux. $\mathrm{HH}$ is associated to the partial or total loss of hepcidin, which results in excessive iron uptake and accumulation in the tissues. $\mathrm{HH}$ patients present anomalies in $\mathrm{CD}^{+} \mathrm{T}$ cells and altered $\mathrm{CD} 4^{+} / \mathrm{CD}^{+} \mathrm{T}$ cell ratios; in particular $\mathrm{HH}$ is associated with lower numbers of $\mathrm{CD} 8^{+} \mathrm{T}$ cells in the circulation and liver and a defect 
in the generation of $\mathrm{CD}^{+}$memory cells [197]. Altered cytokine profiles are also seen in $\mathrm{HH}$ patients, where $\mathrm{CD}^{+} \mathrm{T}$ cells increase the production of IL-10 and IL-4 contributing to Th2 polarization of the adaptive response. Although HFE is a non-classical MHC Ib molecule it does not appear to have antigen presenting capabilities; however, a role for HFE in the antigen presentation process cannot be ruled out. TFR1 interacts with the TCR $\zeta$ chain, which is involved in signal transduction upon antigen recognition [216]. Therefore, by competing with transferrin, and depending on iron availability HFE could regulate $\mathrm{T}$ cell activation. In addition to the altered lymphocyte ratios, some $\mathrm{HH}$ patients may have altered iNKT numbers. Iron overload was associated with reduced numbers of iNKT cells, which was more notable in untreated patients [200].

The balance between iron and selenium also seems important to the development of $\mathrm{T}$ cell responses. Iron metabolism is key to survival of $\mathrm{CD}^{+} \mathrm{T}$ cells as $\mathrm{Fe}^{2+}$ mediates a type of cell death known as ferroptosis in CD8 ${ }^{+} \mathrm{T}$ cells. During ferroptosis, $\mathrm{Fe}^{2+}$ reacts with $\mathrm{H}_{2} \mathrm{O}_{2}$ produced by mitochondrial respiration driving the formation of free hydroxyl radical $(\mathrm{OH} \cdot)$ which promotes lipid peroxidation and death. The glutathione peroxidase $4(\mathrm{Gpx} 4)$ is a selenoenzyme that acts as a major scavenger of phospholipid hydroperoxides and is essential to prevent ferroptosis. Gpx4 is essential for homeostasis of both $\mathrm{CD} 4^{+}$and $\mathrm{CD} 8^{+} \mathrm{T}$ cells, as mice lacking Gpx4 have impaired proliferate $\mathrm{T}$ cell responses during a viral challenge or Leishmania infection [217]. Selenium has been shown to be important for both cellular and humoral immune responses, although cell-mediated immunity seems to depend more on selenium. Selenium can enhance responsiveness of lymphocytes to IL-2 by inducing the upregulation of IL-2 receptors, therefore promoting proliferation, cytotoxic activity and antibody production [218]. High selenium diets promote Th1 differentiation in mice. Whereas selenium deficiency has been linked to more severe influenza in mice [219]. Selenium deficiency leads to reduction in the expression of selenoprotein $\mathrm{K}$ (SelK), which is expressed in the endoplasmic reticulum membrane of many immune cells including $\mathrm{T}$ cells. SelK deficiency impairs proliferation of $\mathrm{T}$ cells as it impairs crucial $\mathrm{Ca}^{2+}$ fluxes upon TCR activation. SelK deficiency also affects neutrophil and macrophage function increasing susceptibility to a viral challenge. Zinc $\left(\mathrm{Zn}^{2+}\right)$ is required for thymic development of $\mathrm{T}$ lymphocytes. $\mathrm{Zn}^{2+}$ deficiency leads to impaired $\mathrm{Th} 1$ responses while promoting Th17 differentiation and IL-1 $\beta$ production [219].

\section{Host nutrient availability and the microbiome of the lung}

The human body harbours over 100 trillion microorganisms that live in a commensal relationship with their host, mostly at barrier surfaces and mucosae including the gut, the airways, skin and genitourinary tract. Imbalances in the composition of the human microbiota have been increasingly linked to pathologies such as allergy, asthma, chronic inflammation and autoimmunity. While the gut has been the most studied and characterized niche in terms of the microbiome, in recent years the importance of other niches including the lung microbiome, became apparent. We are only now learning that the lung is colonized by a complex and dynamic microbiota. While progress in understanding how changes in the lung microbiome contribute to disease has been limited due to sampling, technical and analytical problems, $16 \mathrm{~S}$ sequencing studies have demonstrated distinctive differences in the lung microbiome between health and disease [220]. Despite the low density of the lung microbiota, the extraordinary diversity of interacting microbiota is evident, and it is the change or decline in this diversity that is often associated with the progression of disease. To date, no particular bacterial genera have been implicated in lung disease and the variability in the diversity of the species detected in the upper versus lower respiratory tract as well as the regional variations in the host environment (e.g., mucus or surfactant secretion, $\mathrm{pH}$, nutrient or oxygen availability) has also limited our understanding of the contribution of the respiratory microbiome in lung disease [221].

It appears that the airway microbiota in healthy lungs is dominated by Bacteroidetes and with prominent genera including Prevotella, Veillonella and Streptococcus [222-224], all of which are dependent on metals for growth and survival $[9,225,226]$. Approximately $30 \%$ of all proteins in bacteria depend on metals for their function [226] and changes in host metal availability alters bacterial diversity and abundance in vivo [227-236], however there have been few studies examining the effect of metal availability directly on lung microbe populations [237]. In addition, whether or not changes in host metal availability in the lung directly modifies the lung microbiome which in turn contributes to the development and progression of chronic or acute respiratory disease is an evolving question.

Similarly, it has become evident that it is not only the microbial communities but also the metabolites they produce that can influence host susceptibility to certain diseases [238]. Despite the advances in microbiome research, our knowledge of the airway microbiome is still way behind compared to that of the gut microbiome. And as such, lung microbiota composition, microbiota 
derived metabolites and how they could contribute to lung inflammation, immunity and disease is only in its infancy $[239,240]$. Alterations in bacterial burden, gut microbial species and the metabolites they produce are associated with altered inflammation and immunity in the lungs as well as the development of lung diseases. This interaction, known as the gut-lung axis allows the access of gut-derived bacterial components, hormones, microbial metabolites, endotoxins and cytokines to the lung niche via the bloodstream, also influencing immune cell trafficking. Future research of the lung microbiota in the context of both heathy and diseased airways will likely discover the causes and consequences of altered lung microbiota in lung disease and identify microbial derived metabolites that play important roles in these processes. Whether or not microbe derived metal regulators interfere with the host immune responses in the lung therefore requires significant attention. In the below sections we will discuss the existing evidence for alterations in host metal availability in chronic respiratory disease and the evidence, if any, for the role trace metals have to play in the nutritional immune response or to changes in the lung microbiome.

\section{Nutritional immunity and the host lung microbiome in chronic respiratory disease} Asthma

Asthma is a chronic and heterogeneous disease of the airways characterized by airway hyperreactivity, difficulty breathing, cough, wheezing and chest tightness. It is estimated that 300 million people are affected with asthma and that $\sim 250,000$ die as a result each year. The pathogenesis of asthma is complex with innate and adaptive cells acting together with epithelial cells to induce airway hyperreactivity. A broad clinical spectrum of phenotypes associated to different underlying immune mechanisms (endotypes) have been described [241, 242]. Asthma can be divided in two subsets: the eosinophilic type mainly controlled by Th2 or ILC2s, and the neutrophilic type which is characterized by a strong Th17 component and can present as steroid-resistant asthma. Eosinophilic asthma can be categorized as Th $2^{\text {hi }}$ and Th $2^{\text {lo }}$ component depending on the presence of IL-4, IL-5 and IL-13 being produced and the number of eosinophils in blood and lungs [243]. In addition to Th2 cells, ILC2 can also contribute to the pathology of asthma and produce high amounts of type 2 cytokines. These cells have been identified in samples of blood but also BALF and sputum of asthmatic patients [177]. ILC2 also drive mucus hypersecretion, mucus cell metaplasia, fibrosis and inflammation which are driven by IL-13, eosinophils and monocytes [244]. Late onset and steroid-resistant asthma phenotypes are associated with IL-17 driven neutrophilic asthma and present with a more irreversible airway obstruction. Besides Th17 cells, cell including y $\delta$ T cells, NKT cells and ILC3 can contribute to IL-17A, IL-17F and IL-22 production amplifying the pathology [242, 245].

$\mathrm{T}$ helper responses in asthma are supported by DCs that are directly activated by allergens and other environmental insults or via the damaged epithelium. Allergens including house dust mites, spores, cat dander and others can have protease activity that damages epithelial cells and triggers protease-activated receptors and triggers the release of damage associated molecular patterns (DAMPs) that lead to the secretion of innate responses via IL-33, TSLP, TLR4 and C-type lectin receptors among others [242]. Among lung DCs conventional DC expressing CD11b and SIRP $1 \alpha$ and that rely on interferon regulatory factor (IRF) 4 are the most important for allergic sensitization [246, 247]. DCs and epithelial cells not only contribute to sensitization to allergens but also play a role in ongoing asthma.

\section{The lung microbiome and asthma}

The time from the perinatal period up to the first years of life is key to the establishment of a "healthy" microbiome. The composition of the microbiome at all anatomical sites is dynamically shaped by the interactions between symbionts, pathogens, the immune system, nutrition and the environment. Disturbances during the critical period of establishment of this ecosystem may determine future pathological manifestations [248]. Several retrospective and longitudinal studies suggest this is the case for asthma and airway hyperreactivity, where aberrant immune development results from environmental exposures that may influence the airway microbiota. The analysis of the airway microbiome in infants revealed an association between the airway microbial composition and the risk of developing asthma within the first 6 years of life. Enrichment of the upper airway microbiome with taxa including Veillonella, Prevotella, and Gemella, was associated with increased risk of asthma and a characteristic immune profile in the airway which was also independently associated with increased asthma risk within 6 years. While the study cannot prove causation, it suggests a potential link between an early shift in the airway microbiome composition and immune perturbation which could lead to asthma later in life [249].

Analysis of different respiratory samples in healthy vs asthmatic adults suggests that the lungs of asthmatic patients receiving corticosteroid therapy are dominated by the phylum Proteobacteria which include the genera of potential pathogens including Haemophilus, Moraxella, and Neisseria [250]. Response to steroids in accompanied by an increase in Actinobateria, whereas Kelbsiella is linked to severe asthma [250]. In addition, patients 
with severe neutrophilic asthma receiving high doses of inhaled corticosteroids display lower airway microbiome diversity, enrichment with Moraxella and Haemophilus and a reduction in Streptococcus, Gemella and Porphyromonas [248]. While airway microbiome profiles seem to correlate with severity of the disease and may even predict response to corticosteroids, it is not possible to discriminate whether these changes are promoted by the interactions between commensals and the immune system or induced by the steroid treatment. In a group of steroid-naïve asthmatic patients, members of the Sphingomonodaceae family and species of Haemophilus, Neisseria, Fusobacterium, and Porphyromonas were enriched in the bronchi compared to healthy controls, whereas members of the Mogibacteriaceae family and Lactobacillales order were lower. Importantly, steroid treatment caused a shift in the balance of the bronchial microbiome and differences were linked to responsiveness to treatment [251].

In addition to the local airway and lung microbiome, the distant gut microbiome and the gut-lung axis of immune regulation have been implicated in the pathogenesis of asthma. Significant reduction in species of the genera Lachnospira, Veillonella, Faecalibacterium and Rothia in the gut of 3-month-old infants correlated with elevated risk of developing asthma [252]. Mechanistically, high risk of asthma and gut dysbiosis has been linked to loss of short-chain fatty acid (SCFA)-producing bacteria which ferment soluble fibre into these highly volatile metabolites (e.g., acetate, propionate, butyrate) known for their immunoregulatory properties [253] Higher levels of butyrate and propionate in the stool of 1-year old toddlers associate with less atopic sensitization and low risk of asthma between the age of 5 and 6 [254] and supplementation with soluble fibre inulin, associates with clinical improvement of asthma in adults and an increase in Bifidobacteria which degrade inulin into the SCFA acetate and lactate [255].

Intestinal bacteria can also produce metabolites that may be detrimental for lung function. For example, histamine-producing bacteria are more abundant in the gut of asthmatic patients compared to healthy controls and this histamine has been liked to airway hyperrreactivity [248]. Shifts in the microbial populations in the airways and in the gut seem to be associated with asthma endotypes and severity, the causal link between development of the disease and dysbiosis remains to be proven. The current evidence supports mechanisms of immune modulation mediated by microbial-derived metabolites. While an inverse correlation between SCFA in the gut and asthma severity exists, whether significant concentrations of SCFA can reach the bloodstream or the lungs remains questioned. It would be interesting to determine if changes in the lung microbiome influence local production of these metabolites to directly modulate the lung response to allergens and other environmental insults that could promote asthma.

\section{Trace metals, nutritional immunity and asthma}

Epidemiological studies have shown a correlation between iron deficiency and atopic disease, involving iron metabolism in the regulation of the immune response to allergens and atopic airway hyperreactivity [256, 257]. A large cross-sectional study in children and adolescents in the US, found a strong association between anaemia and atopic disease, including eczema or asthma [257]. The prevalence of asthma decreases with age and the iron reserve is higher in adults compared to children supporting the idea that the higher incidence of asthma and atopic disease in early life is linked to iron deficiency. In adulthood, men have higher levels of iron compared to women and asthma primarily affects women, again supporting a role of iron in prevention of asthma [41]. Though, the relationship between iron and asthma is not straightforward: increased iron stores in the form of ferritin have been associated with decreased odds of asthma, whereas higher tissue iron (lower serum soluble transferrin receptor) and lower body iron have been linked to lower lung function [256].

The iron storage capacity of Th2 cells is higher than Th1 as the latter have a lower iron labile pool [258]. Iron chelation affects Th1 cytokine production including IFN-y, IL-12 and IL-18, but not Th2 cytokines [259]. Therefore, a limited iron supply during stimulation of lymphocytes by allergens may favour development of Th2 responses over Th1 and predispose to allergic sensitization [41]. Severe and moderate asthma patients present low cell-free (non-heme) iron levels in BALF which correlate with lower lung function measured as (forced expiratory volume in $1 \mathrm{~s}$ (FEV1), whereas iron-loaded cells numbers are increased and also show increased expression of DMT1 and TFR1. These features were recapitulated by a murine model of house dust mite asthma where macrophages display the highest expression of Tfr 1 and also upregulate Il13, suggesting that by accumulating iron they orchestrate the type 2 response in the lungs [260]. The relationship between iron metabolism and pathophysiology of asthma represents a dichotomy: while low systemic and local iron levels in BALF correlate with severity of asthma, cellular iron overload is also linked to the pathology of the disease.

Importantly, most allergens of mammalian origin including those in dander, urine, fur, and saliva of animals, belong to the lipocalin family of proteins and have the capacity to sequester bacterial siderophoreiron complexes. Some of these allergens promote Th2 
responses when not bound to iron, suggesting that iron deficiency may potentiate the stimulatory potential of lipocalin-like allergens [261, 262]. In addition, to promoting Th2 responses directly, allergens may change the microbial ecosystem of the gut and/or the airways by sequestering bacterial siderophores and altering the availability of iron to certain bacteria, therefore inducing dysbiosis. Supporting this notion, supplementation of dietary iron in rats favoured the production of the SCFA butyrate compared to animals fed an iron deficient diet [263]. This suggests that iron availability can influence the composition of the microbiome and the production of immunomodulatory metabolites such as SCFA that are important modulators of type 2 allergic responses and asthma [41].

The role of other metals in the pathophysiology of asthma is less clear but serum concentrations of trace elements including copper, zinc and selenium have been found to be altered in individuals suffering from asthma compared to healthy controls. Imbalances in selenium have been implicated in allergic inflammation. Curiously, while mice fed on high and low selenium diets show low incidence of allergic inflammation in an ovalbumin sensitization model, intermediate doses of selenium were associated with heightened inflammation. This suggests that the modulation of the allergic response by selenium in the lungs is not linear and may involve several intermediate enzymes and other unknown mechanisms [264].

Two independent studies reported elevated serum levels of copper in adults suffering from asthma compared to healthy controls, whereas zinc and selenium were lower and magnesium remained unchanged [155, 265]. While the mechanisms by which alterations in trace metal concentration contribute to the pathology are unclear, given the role of copper and zinc in regulation of redox metabolism, altered levels of these metals may contribute to imbalances in oxidative stress in asthma. Indeed, asthmatic patients present higher levels of NO and nitrated products than healthy individuals, and these correlate with the severity of the disease. Non-enzymatic decomposition of S-nitrosothiols (RNSO) is mainly catalyzed by $\mathrm{Cu}^{2+}$ ions leading to generation of $\mathrm{NO}$ and the corresponding disulphide [266]. Therefore, it is possible to postulate that elevated copper levels in asthma may accelerate RSNO consumption with the concomitant increase in NO. Supporting this hypothesis, RSNO deficiency in the airways has been linked to asthmatic respiratory failure in children which suggests that RSNO metabolism and in particular their copper-mediated catalytic decomposition could be therapeutic targets in asthma [267].

\section{Chronic obstructive pulmonary disease}

COPD is a chronic inflammatory lung disease associated with cigarette smoke or other environmental exposures. As a leading cause of death worldwide, COPD encompasses chronic bronchitis and emphysema [268, 269] and involves an aberrant immune and inflammatory responses to the inhalation of noxious particles [269, 270], with chronic inflammation persisting for many years after [204]. The definitive molecular mechanisms underlying the chronic inflammatory changes observed in COPD remain to be determined, however there is overwhelming evidence implicating AMs in this process [204, 271, 272]. AMs exposed to cigarette smoke have been shown to ineffectively clear respiratory pathogens, damaged epithelial cells as well as having defective responses to activating stimuli [273-276]. Strikingly, there is a 25-fold increase in the number of AMs in the lung parenchyma and alveolar space in COPD, which correlates with disease severity $[277,278]$ and areas of lung destruction [272], highlighting their central role in disease pathogenesis. Neutrophilic inflammation is also a hallmark of COPD and is associated with bacterial infection [279] whereas eosinophilic inflammation is present in a subgroup of COPD patients [280], and is associated with less bacterial infection [281]. Aberrant regulation of the immune response is a clear factor in the development and progression of COPD however little is known regarding the microbiome or nutritional immunity in this process.

\section{The lung microbiome and COPD}

Microbiome changes have emerged as a contributing factor in COPD progression, clinical phenotypes, severity and long-term mortality [282-284]. Studies comparing sputum and BALF microbiota between stable COPD patients and healthy controls have identified a change in microbial diversity with an increased abundance of Moraxella, Streptococcus, Proteobacteria, Veillonella, Eubacterium and Prevotella in disease [283-289]. Other studies of BALF report no difference in Streptococcus [290]. Conversely, increased Proteobacteria and reduced Firmicutes, Bacteroidetes, Streptococcus, Haemophilus influenza and Prevotella spp. have been documented in COPD lung tissue explants [291]. During an exacerbation event (periods of symptom worsening and reduced lung function) shifts in bacterial composition, characterized by a relative increase in Proteobacteria that falls in response to antibiotics have also been observed [292, 293]. Some of the above studies suggest that these changes may be independent of smoking status and that real changes in microbial diversity may be more apparent upon examination of associations in specific COPD endotypes [289] such as the neutrophilic inflammatory 
endotype $[283,294]$. Intriguingly the faecal microbiome and COPD patients is also distinct from those of healthy individuals, with Streptococcus sp000187445, Streptococcus vestibularis and multiple members of the family Lachnospiraceae correlating with reduced lung function [295]. During an acute exacerbation event the faecal microbiome has also been shown to display a lower relative abundance of Firmicutes and Actinobacteria with an increase in Bacteroidetes and Proteobacteria [296]. The importance of the gut microbiota in COPD development is further highlighted by the reversal of murine smokeinduced inflammatory emphysema via faecal microbiota transplantation or a high-fibre diet, possibly via the beneficial effects of SCFAs [297].

\section{Trace metals, nutritional immunity and COPD}

Genome wide association studies of COPD patients implicate a role for abnormal iron metabolism in COPD [298-302]. Current and former smokers have abnormally high levels of iron in sputum, BALF in exhaled breath condensate, and in AMs, compared to non-smoking controls [211, 303-312]. Anaemia and non-anaemic iron deficiency often accompany COPD [313] with anaemia being an independent predictor of mortality [314-316], and with iron-deficient COPD patients having more exacerbations than control subjects [317]. Smoke exposure also reduces hepcidin expression in murine models. COPD patients have an inappropriate suppression of hepcidin in response to iron deficiency, with less hepcidin expression in severe end-stage disease $[54,316,318]$. This may or may not be related to findings that iron accumulates inside cells and tissues of in vitro and in vivo COPD models [304, 305, 309]. Whether increased cellular iron is pathogenic [319] or a protective [307, 320] stratagem should consider the lung and systemic iron regulation as separate entities. Both the use of iron chelators directly in the lung as well as administering intravenous ferric carboxymaltose to iron deficient COPD patients may have beneficial effects [319, 321].

As previously mentioned, iron plays an important role in the functional response of macrophages and other immune cells of the lung and similarly iron regulatory pathways play an important role in the response of these cells to smoke [54, 304, 319]. Specifically, cigarette smoke increases the expression of FPN, ferritin and the TFR1 on AMs and inhibits hepcidin induction by LPS as well as inducing ferroptosis [54, 322]. However, whether or not the above changes in macrophage iron regulation in the lung as a result of smoke or COPD progression alters the interplay between immune cells and the lung microbiome remains to be determined.

Zinc levels have also been shown to play an important role in COPD. COPD patients have lower levels of zinc
$[77,323]$ and smokers with low dietary zinc intake have an increased incidence of COPD [324]. The zinc transporter ZIP8 is also increased at mRNA and protein levels in the lungs of chronic smokers [325] and zinc deficiency potentiates the effects of smoke on the epithelial cell barrier function [326, 327]. Depletion of zinc in adult mice resulted in a significant increase in lung cadmium burden and permanent lung tissue loss following prolonged smoke exposure [328]. Zinc also regulates the immune response to smoke whereby airway inflammation is exaggerated in zinc deficient mice [329] and zinc supplementation reduces AM numbers in smoke-exposed mice [330]. Zinc protects against cadmium toxicity and loss of zinc in AMs leads to the toxic accumulation of cadmium in the AMs of smokers [331]. Functionally, loss of zinc inhibits efficient efferocytosis of apoptotic epithelial cells by AMs [77]. Whether or not a loss in zinc levels alters the microbiome of the lung or the response of immune cells such as AMs to infection remains to be determined.

Copper also plays a role in the development of COPD. Copper deficiency induces emphysema in animal models [332-334] and the EBC of stable COPD patients contains lower copper levels than of controls an observation that is positively related to FEV1 in individuals with COPD [335]. Intriguingly, individuals with Menkes disease, an $\mathrm{X}$-linked recessive disorder of mutations in the intracellular copper-transporter ATP7A display an increased incidence in emphysema [336, 337]. A loss of copper directly impacts AEC integrity and copper directly regulates elastin synthesis via the copper dependent enzyme lysyl oxidase [334, 338]. However, whether or not copper levels in lung immune cells dictate function or regulate responses to cigarette smoke remains to be determined. Finally, selenium responsive genes are altered in individuals with COPD [339] and COPD patients with higher selenium have a higher FEV1 [340]. Selenium and manganese levels may be beneficial before the clinical onset of COPD in smokers [341] and manganese levels are higher in COPD patients with severe disease when compared to control smokers [342] however the role of selenium or manganese in disease pathogenesis is unknown.

\section{Cystic fibrosis}

Cystic Fibrosis (CF) is an autosomal recessive disease caused by mutations in the CF transmembrane conductance regulator gene (CFTR). The CFTR gene encodes a protein that mainly acts as a chloride channel that transports ions across the apical membrane of epithelial cells. This disease is characterized by progressive lung disease, malnutrition, growth defects and several other presentations [343]. In the lungs, CF is characterised by mucus accumulation and obstructive lung disease. Reduced mucus clearance leads to higher bacterial loads in the 
lower airways and recurrent respiratory infections are the main cause of morbidity and mortality in CF patients, with Pseudomonas aeruginosa being one of most common pathogens in CF.

\section{The lung microbiome and $C F$}

The altered landscape of the CF airways results in a dysbiosis of the airway microbiome, however which specific factors of the CF lung microenvironment drive this change in the microbiota still remains unknown [344]. The onset of the CF microbiome involves an increase in bacterial burden and a reduction in microbial species diversity. Infants and children with CF have a higher microbial diversity which is lost with age, disease progression, and the domination of CF pathogens, most notably $P$. aeruginosa [345-349]. In advanced disease the lower airways consist of mostly homogenous populations of CF pathogens [350]. The main taxa found within CF microbiomes are Streptococcus, Prevotella, Veillonella, Rothia, Actinomyces, Gemella, Granulicatella, Fusobacterium, Neisseria, Atopobium and Porphyromonas [351, 352]. Diversity appears to serve as a marker for lung function as reduced species richness correlates with reduced lung function [351,353]. Several studies have examined the airway microbiome in CF patients during exacerbation $[351,354,355]$ and have observed that overall microbial community structure remains the same during stable periods and exacerbations and that the extent of changes observed during exacerbation are dependent on the community composition and diversity at baseline. In one study the relative abundance of Gemella was increased in most of the patients during exacerbation and was the most altered genus between baseline and exacerbation [354]. In this same study a subset of patients had substantial changes in microbial community structure during exacerbation in that those that had Pseudomonas dominated microbiota communities at baseline became more diverse at exacerbation. Furthermore, the presence of anaerobes in the microbiota is associated with reduced inflammation and higher lung function at early exacerbation compared to Pseudomonas [356]. While many studies have identified the constituents of the microbial community in the CF lung and during exacerbation and stable periods, there are few mechanistic insights into how these microbial residents of the airways can contribute to this disease. A recent study quantified bacterial active translation in the sputum from $\mathrm{CF}$ patients and found that active bacteria (i.e., those actively translating proteins) represent only a subset of those captured by conventional sequencing [357]. By adopting a dormant phenotype in which reduced cellular activity endows a temporary multi-drug resistant phenotype, the inactive bacteria subpopulations could persist during antibiotic challenge. While the common residents of the CF airway microbiota are now known, their physiology and how they interact with other members of the microbiota and with their airway microenvironment remain to be more fully explored in order to understand how CF drives altered microbial community in the airways and how the CF microbiota subsequently contributes to CF disease progression.

\section{Trace metals, nutritional immunity and CF}

Systemic iron deficiency is prevalent among adults and children with CF [358-364]. However iron levels are elevated in the airways in CF with higher iron levels detected in the sputum and within AM and IMs [365-368]. With macrophages being key regulators of extracellular iron levels, they represent a likely source contributing to increased airway iron. Indeed, CF macrophages have recently been shown to have altered iron metabolism that can be corrected with CFTR modulators ivacaftor and lumacaftor [369]. Furthermore, airway epithelial cells expressing $\triangle \mathrm{F} 508-\mathrm{CFTR}$, the most common CFTR mutation observed in CF, have been shown to have altered iron homeostasis and release more iron than those expressing WT-CFTR [370, 371]. This increased iron in the airways provides easier access of this vital nutrient to bacteria present in the airways, including $P$. aeruginosa. Iron not only is essential for growth, but it also regulates biofilm formation in this respiratory pathogen $[369,370,372]$. This has a major impact on the ability of these bacteria to persist in the lungs as bacteria within biofilms are much more difficult to clear by host immunity and by antibiotic treatment. With persistent $P$. aeruginosa lung infections driving increased mortality in $\mathrm{CF}$ patients, reducing high iron levels in the lungs or depriving $P$. aeruginosa of iron appears to be a sensible strategy to control this CF pathogen. Gallium has been used to treat $P$. aeruginosa in CF, this metal was shown to and inhibit growth of $P$. aeruginosa in sputum and improved lung function in people with $\mathrm{CF}$ and chronic infection by interfering with bacterial iron metabolism [373]. Gallium compounds have also been shown to inhibit in vitro and in vivo growth of Mycobacterium abscessus by disrupting iron uptake in this emerging important pathogen in CF[374].

Zinc deficiency has been observed in CF patients, especially those with malabsorption and impaired growth. The serum or plasma levels of subsets of CF patients, both child and adult, have been observed to be lower than healthy controls in several studies [375, 376]. Zinc supplementation has been used to treat CF patients, but studies have shown conflicting results, some have shown that zinc supplementation leads to improved lung function and reduced need for antibiotics and 
hospitalisations, while others showed no benefits for zinc supplementation [377-381]. Interestingly, while zinc levels in serum can be low, zinc levels in the airways of CFs are reported to be higher [382, 383]. The prevalence and the impact of altered zinc homeostasis in $\mathrm{CF}$ is not yet well defined, however zinc levels affect microbial community structure in CF. Explants of CF lungs had S.aureus and $P$. aeruginosa co-existing in calprotectin enriched regions. As calprotectin chelates zinc, regions in the lungs enriched with this protein will have lower zinc levels which causes $P$. aeruginosa to reduce its production of antistaphyloccal factor thus allowing for the co-existence of this bacterial species [384].

Studies have found reduced activities of copper enzymes from cells (mononuclear cells, neutrophils and erythrocytes) in CF patients, possibly indicating reduced copper availability within these cells $[385,386]$. Interestingly copper supplementation does not counteract this copper deficiency in CF patients indicating an altered copper metabolism that cannot be corrected simply by supplementation [387]. One study reported that copper levels are elevated in the sputum in CF [382]. The cause and consequences of altered copper homeostasis in $\mathrm{CF}$ are yet to be determined. Selenium deficiency is observed in CF patients [388-391] but the levels of selenium in the CF lung have not been reported.

\section{Non cystic fibrosis (CF) Bronchiectasis}

Bronchiectasis refers to the permanent widening of the bronchi and usually presents clinically with coughing, sputum production and recurring respiratory infections, along with other symptoms. The widening of the bronchi leads to impaired mucociliary clearance and failure to effectively clear microbes and mucus leading to persistent infection and inflammation. Bronchiectasis is complex and heterogenous and can be the final common feature of many infectious, inflammatory, and allergic disorders [392].

\section{The microbiome and bronchiectasis}

Several studies of the lung microbiome in bronchiectasis have observed alterations. A longitudinal study of non$\mathrm{CF}$ bronchiectasis patients found that the airway microbiomes remained stable over time and that patients with lower diversity of the microbial community were more likely to experience a subsequent decline in lung function [393]. This study also reported that the microbiomes of patients that were dominated by Pseudomonas differed greatly from patients whose microbiomes are Haemophilus dominated and that antibiotic treatment did not affect their microbiome composition. However, a larger study observed that disease severity is reduced with lower microbiome diversity [394]. Another study reported no significant changes in microbial diversity and during or after exacerbations, but also observed stability in the microbiomes of patients over time ( 6 months) even with antibiotic therapy [395]. This study also highlighted the differences in culture versus sequencing to identify the composition of the airway microbiota, notably $H$. influenzae is not detected well by culture. A study which compared bronchiectasis patients treated with low dose of a macrolide antibiotic (erythromycin) with a placebo control found that patients with Haemophilus dominated microbiomes were associated with fewer exacerbations but both Pseudomonas and Haemophilus dominated microbiomes were associated with lower lung function [396, 397]. Infections caused by non-tuberculous mycobacteria (NTM) are often seen in patients with bronchiectasis. These bacteria are found in the environment and usually do not cause disease but can establish chronic infections in people with underlying conditions. The role of NTM in the lung microbiome and how it may interact with other species in the microbiota of bronchiectasis patients, or even in healthy individuals, is not yet known. Limitations of sequencing approaches commonly used contribute to this lack of knowledge and studies have shown that $16 \mathrm{~S}$ rRNA gene sequencing is not sensitive enough for Mycobacteria [398, 399]. Using a more sensitive technique for mycobacteria, a study was able to identify a non-tuberculous 'mycobacteriome' in in the mouth and upper respiratory tract of healthy individuals [399]. A study involving non-CF bronchiectasis patients with high prevalence of NTM used this mycobacteriome sequencing approach for the lower airways of 20 patients [398]. They found that in the lower airways of NTM positive patients, taxa identified as oral commensals were associated with increased inflammatory biomarkers. Studies with larger cohorts and using sensitive sequencing methods for mycobacteria are required to further investigate the role of NTM in the microbiome, particularly in the context of bronchiectasis.

\section{Trace metals, nutritional immunity and bronchiectasis}

The roles that metals such as iron, zinc and copper may play in non-cystic fibrosis bronchiectasis is not known and there are only a few reports measuring the levels of these metals in patients with this chronic lung disease. Some reports have observed that altered trace metals levels in non-CF bronchiectasis patients. Specifically, serum zinc levels have been reported to be lower in bronchiectasis patients when compared to healthy controls, but an earlier study did not find significant differences in serum zinc levels and found that even though zinc supplementation resulted in increased zinc serum levels no clinical improvement was observed [400, 401]. Two studies have detected higher levels of iron and zinc in the sputum of 
non-CF bronchiectasis patients compared to controls $[382,383]$. Bacteria that cause recurrent infections in non-CF bronchiectasis could benefit from easier access to essential trace metals in the airways, supporting their growth. Thus further study of the source of these trace metals in the airways and their role in both the pathology of non-CF bronchiectasis, and in respiratory infections in those with chronic lung disease needs to be further investigated.

\section{Lung cancer}

Lung cancer is one of the most aggressive and lethal cancers, with low treatment success and many complications. The most common form of lung cancer is nonsmall cell lung cancer which is subdivided into three main types; adenocarcinoma (AD) which arises in the peripheral bronchi, squamous cell (SC) which arises in the main bronchi and large-cell undifferentiated carcinoma [402]. The less common form of lung cancer is small cell lung cancer (SCLC) which accounts for $15 \%$ of lung cancers is characterised by its neuroendocrine features [403]. Previously, lung cancer was thought to be non-immunogenic due to failed immunotherapies but has recently been revisited as being correlated with various immune cells and responses, particularly with inflammatory responses being integral to cancer progression [404]. Commonly during early stages of lung tumour generation an influx of immune cells such as leukocytes into the tumour and around the tumour microenvironment (TME) are observed [405]. The main source of this immune inflammation comes from M2 macrophages, which have been shown to promote angiogenesis, tissue remodelling and repair for the malignant cells [406].

\section{The microbiome and lung cancer}

Studies testing patients with lung cancer found strong associations between the presence of malignancy and high densities of Haemophilus influenzae, Acidovorax, Klebsiella, Moraxella catarrhalis, Mycobacterium tuberculosis and Granulicatella adiacens [224, 407]. Furthermore, comparison between the taxa present in SCLC and AD revealed specific taxa such as Acidovorax, Klebsiella, Rhodoferax, Tepidimonas and Anaerococcus were more abundant in SCLC [408]. Possibly through an environmental influence, the density of commensal bacteria was reduced within the lungs, allowing for more insidious opportunistic species to proliferate and drive lung inflammation. Microbial profiles have been suggested as a potential biomarker for lung cancer. Using 16S RNA sequencing and real time PCR Yan et al. (2015) successfully linked microbiota present in the saliva to lung cancer. Both Capnocytophaga and Veillonella were significantly higher in patients with lung cancer [409]. However, the sample size of this study was relatively small so further investigations into the affiliation between microbial composition in saliva and lung cancer is needed. In more recent studies, comparisons of the microbial profile between patients with emphysema and or lung cancer noted that the microbial composition of lung cancer tissue samples were distinctly different from both controls and patients with emphysema [410]. Furthermore, preliminary analysis of the microbiome in the sputum identified six further bacterial species that were significantly more abundant in lung cancer samples compared to controls [411]. It is clear that there is link between microbial composition and disease status, however, it is noted that the above studies attempted to analyse the lung microbiome indirectly through analysing the microbial composition in either saliva or sputum. Further direct analysis of the lung microbiome are needed to establish a stronger link between the microbial profile within the lung and lung cancer.

The gut microbiome has also been identified to play potential roles in the pathobiology of lung cancer. For example, H.pylori has been suggested to play a role in lung cancer pathology $[412,413]$ with patients with lung cancer possibly having a significantly higher rate of seropositivity for antibodies against H.pylori [414], however these findings have not been well replicated $[415,416]$. The mechanisms behind bacterial burden and lung cancer include; the production of bacterio-toxins and proinflammatory factors, which could ultimately lead to the DNA damage and mutation causing malignancy, as well as a hyperinflammatory immune response [417, 418]. Also of note, evidence from patients with lung cancer has suggested antibiotic use during treatment to have a largely negative effect on tumour regression, with antibiotics only strengthening the pathogenic bacteria and staving off healthy commensal species [419]. Taken together it is clear that both commensal and infiltrating bacteria play a strong role in the carcinogenesis of lung cancer. Further investigations should attempt to directly assess the lung microbiome, for example, through BALF samples collected via endotracheal tube extraction to avoid contaminants from both mouth and upper airways [349].

\section{Trace metals, nutritional immunity and lung cancer}

Disturbed metal homeostasis has been previously proposed as a biomarker of lung cancer [420]. Specifically, increased level of copper and decreased level of zinc in the serum has been a successful indicator for lung cancer when compared to healthy controls [421]. Increased serum copper may aid in tumour progression through angiogenesis, while decreased zinc may lead to unprecedented changes in cell cycles and apoptosis [422]. In 
contrast, increased plasma zinc levels correlated with a lower risk of lung cancer [423]. Higher levels of zinc were also shown to regulate eight different cancer genes and suggested to prevent cancer progression through decreasing telomere attrition [423].

Iron accumulation has been seen in several cancers and has even been suggested as a potential target for cancer therapy [424]. In the context of lung cancer, ferritin levels in patients are significantly elevated when compared to controls and patients with COPD [425]. Increased expression of other iron regulators like LCN2 and TFR1 have also been observed in lung cancer patients [426, 427]. Furthermore, down-regulation of LCN2 or TFR1 in murine models for adenocarcinoma was shown to significantly suppress the growth of the tumours [426, 427]. Higher IL-6 levels in lung cancer patients [428] may be associated with the upregulation of hepcidin, resulting in decreased iron influx and induced cancer-related anaemia [429]. miR-20a, upregulated in non-small cell lung cancer (NSCLC), and can negatively regulate FPN, which may promote proliferation of cancer cells indirectly through the retention of iron [430]. Iron has also been reported to induce cancer stem cells and aggressive phenotypes through ROS generation in human lung cancer cells [431].

The TME has been characterized as a key player in tumour growth and progression [432]. Tumour associated macrophages (TAMs) are the most commonly found immune cell in the TME and have been shown to integrate an M2 'like' phenotype to promote tumour growth and proliferation [405]. In contrast, a subset of TAMs, iron-loaded TAMs (iTAMs), were characterized as tumoricidal due to their ability to switch the polarization of M2 macrophages to pro-inflammatory M1 macrophages when exposed to iron or heme [433, 434]. Furthermore, AD patients with higher levels of iTAMS were shown to have an overall better survival in comparison to controls [435]. Little is known about where the surplus of iron is coming from, although some studies have suggested cigarette smoking may be a contributing factor [304]. Metagenomic sequencing of microbial profiles in the sputum of lung cancer patients have also identified increases in iron siderophore sensors and receptors [411]. M2 'like' TAMs produce higher levels of FPN and lower levels of ferritin when compared to M1 macrophages. Furthermore, it was suggested that M2 'like' TAMs contribute to tumour progression through their "iron-recycling" phenotype and fulfil the high iron demand from tumour cells by exporting iron into the TME [433]. Interestingly, the treatment of cells with exogenous iron can prevent liver metastases by inducing a pro-inflammatory $\mathrm{M} 1$ polarization in macrophages [436].
Links between other trace-elements, such as selenium and manganese with cancer have also been explored in the literature. While there are no reports on altered levels of manganese in lung cancer, some studies have reported an increase in manganese SOD (MnSOD) activity to be correlated with the disease [437-439]. Genetic analysis demonstrated that polymorphisms in the MnSOD gene were shown to contribute to a higher risk of lung cancer [440]. It is thought that these polymorphisms accelerate tumour progression due to the reduced ability to defend against oxidative stress. In more recent studies, over expression of an isoform of MnSOD in cancer cells was shown to increase tumour invasion in vitro [441]. The altered expression and mutations of MnSOD seen in lung cancer implicate an important role for mitigating oxidative stress in order to supress tumour progression.

Finally, the role of selenium in the pathogenesis and progression of lung cancer is controversial with some studies demonstrating a lower risk of lung cancer with higher exposure to selenium [442] and others reporting no correlation between dietary selenium and lung cancer risk a [443, 444]. Further metallomic and observational analysis are needed to clarify whether these metals are associated with the pathogenesis of lung cancer.

\section{Idiopathic pulmonary fibrosis}

Idiopathic pulmonary fibrosis (IPF) is a form of interstitial lung disease, characterised by a gradual, irreversible decline in lung function [445]. Symptoms include gradual dyspnea and a non-productive cough. The disease is diagnosed via $\mathrm{CT}$ or lung biopsy to show the presence of usual interstitial pneumonia. As 'idiopathic' would suggest, the exact cause of the disease is unknown. IPF tends to affect those aged 60 and above, often with a history of smoking, and is more common in men than women. Environmental, occupational and genetic risk factors may also play a role in the onset of IPF. It is thought that persistent damage and abnormal repair to the alveolar epithelium drives the disease. Treatments for IPF are limited, and none can reverse the fibrosis. The current strategy focuses on slowing the progression of the disease and two antifibrotics have been approved, nintedanib and pirfenidone [446].

\section{The microbiome and IPF}

Active infection in patients with IPF is a high risk for mortality, and as antibiotics can improve quality of life for IPF patients, this would suggest that the microbiome can negatively affect disease progression [447, 448]. One study showed bacterial burden in BALF from IPF patients to be twice that of healthy controls, and this increase has been shown to correlate with worsening disease progression $[449,450]$. The microbiota of IPF patients has 
been shown to be less diverse than healthy controls, with increased number of Streptococcus, Haemophilus, Neisseria, and Veillonella [449]. The Streptococcus and Staphylococcus taxa in particular may be linked to increased mortality, though subsequent studies have not supported this [449, 451, 452]. Instead, it may be the increased bacterial load, rather than particular species that is detrimental [449]. Interestingly, germ free mice had reduced mortality in a bleomycin-induced pulmonary fibrosis model despite similar levels of fibrosis, with the authors of the study speculating that the microbiome may alter the balance between Th1 and Th2 responses [450]. In a study comparing the difference in the microbiomes of patients with either stable IPF or those undergoing an acute exacerbation, those with stable IPF had a reduced bacterial load in their BALF fluid [452]. With increased bacterial load comes epithelial cell damage and increased expression of pro-inflammatory cytokines leading to inflammation and fibrosis [453]. Genetics may play a role determining the increased bacterial burden. Mucin 5B is one of a family of proteins that when combined with water forms mucus. Individuals with a polymorphism in the promoter region of the $M U C 5 B$ gene, a gene associated with susceptibility to IPF have a higher bacterial burden than those lacking this polymorphism [449].

As bacteria must acquire metals essential for their viability from their host the altered metal profile in the lung during IPF, as discussed below, may affect bacterial growth. Streptococcus pneumoniae, for example, requires manganese and iron from its host, while high levels of copper are toxic [454]. Growth of both Haemophilus influenzae and $H$. parainfluenzae can be inhibited by the addition of the iron chelator, deferoxamine, to the growth media [455]. Whether the changes seen in metal availability in the lung drive infection during IPF, or whether they occur due to infection or the disease itself is unknown. Further study would be required, but as metal availability can affect the growth of the bacterial species that may exacerbate IPF, monitoring metal levels in BALF may be an indication of how susceptible patients with IPF are to bacterial infection.

\section{Trace metals, nutritional immunity and IPF}

Exposure to different metals, whether in an environmental or occupation settings, has been linked to the development of lung fibrosis and is considered a risk factor in the development of IPF [456]. The availability and metabolism of several metals have also been implicated in IPF. BALF from IPF patients showed lower levels of chromium, nickel, manganese and zinc when compared to healthy controls, and the authors of this study speculated that this may due to increased oxidative stress as these metals are involved in the antioxidant response
[457]. Of the metals implicated in IPF, iron has been the most widely studied. Iron homeostasis is known to be altered in IPF patients as increased iron deposition is seen, which may also lead to increased oxidative stress in the lung $[458,459]$. The same study also showed iron and copper to be elevated in BALF of IPF patients [457].

AMs may play a role in driving fibrosis [460]. Iron accumulation in macrophages leads to persistent activation in other diseases, and as iron is known to accumulate in AMs this may also contribute to damage in IPF [461]. BALF cells from IPF patients have increased irondependent ROS generation [461]. A higher proportion of AMs in IPF patients lack expression of TFR1, when compared to healthy controls [462]. Consequently, a higher level of transferrin is found in BALF and TFR $1^{\text {neg }}$ AMs were shown to be unable to take up transferrin. The inability to sequester iron may lead to an increase in bacterial growth in the lung, for example Staphylococcus aureus can use transferrin bound iron as a growth factor [463]. The impairment in phagocytosis seen in these TFR1 ${ }^{\text {neg }}$ AMs may also allow for increased bacterial growth. Coupled with this possible increase in bacterial burden due to a lack of TFR 1 expression, the TFR $1^{\text {neg }}$ AMs also had an altered gene expression profile that could be described as profibrotic. This increased proportion of TFR $1^{\text {neg }}$ AMs was also linked to reduced survival in IPF patients [462].

Other metalloproteins may also contribute to fibrosis. Lysyl oxidase-like 2 (LOXL2), a copper-dependent monoamine oxidase, has been linked to pulmonary fibrosis [464]. LOXL2 plays an essential role in matrix remodelling and fibrogenesis, but in both bleomycin treated mice and patients with IPF, LOXL2 expression is increased $[465,466]$. The transforming growth factor (TGF)- $\beta$ / Smad signalling pathway has been implicated in the onset of IPF, and signalling through this pathway in fibroblasts from bleomycin challenged mice was reduced when LOXL2 was silenced with siRNA [464]. Targeting LOXL2 with an inhibitory monoclonal antibody has also been shown to be protective in bleomycin exposed mice [467]. SODs convert superoxide radicals into the less damaging hydrogen peroxide and oxygen [468]. Mammalian cells possess three isoforms: the cytosolic copper-zinc SOD1, the mitochondrial manganese-dependent SOD2, and the extracellular copper-zinc SOD3. Macrophages from patients who had been exposed to asbestos have high levels of the copper, zinc-SOD/SOD1, and produce high levels of $\mathrm{H}_{2} \mathrm{O}_{2}$, while SOD $1^{-1-}$ mice were protected from developing pulmonary fibrosis following intratracheal exposure to asbestos [469]. Follow up studies showed that SOD1, via its generation of $\mathrm{H}_{2} \mathrm{O}_{2}$, drives a M2 macrophage phenotype among AMs, which is associated with the development of fibrosis $[469,470]$. 


\section{Pulmonary arterial hypertension}

Pulmonary arterial hypertension (PAH) is characterized by increased pulmonary arterial pressure associated with remodelling of the pulmonary arteries that leads to right ventricular hypertrophy and failure increasing the incidence of right heart failure and death [471]. Variants of pulmonary hypertension (PH) affect over one hundred million people globally [472]. Immune cells play an important role in the development of PAH supported by the observation that dysregulated lymphocytes, AMs, DCs, and mast cells frequently accumulate in the perivascular regions and in the pulmonary arterial vascular lesions found in human PAH tissue samples [473].

\section{The microbiome and PAH}

There have been few studies assessing the airway microbiome in individuals with PAH. However, a recent study described the difference in abundance of microbiota in pharyngeal swabs showing a significantly higher proportion of Streptococcus, Lautropia, and Ralstonia in patients with $\mathrm{PH}$ than reference subjects [474]. Gut microbiota differences have also been noted in PAH and experimental models for PAH have documented alterations in faecal microbiota composition with rodents displaying a three-fold increase in Firmicutes-to-Bacteroidetes ratio $[475,476]$.

\section{Trace metals, nutritional immunity and $P A H$}

Iron deficiency and/or anaemia is prevalent in $\mathrm{PH}$ patients, significantly affecting morbidity and mortality of $\mathrm{PH}$ patients $[477,478]$. Similarly $\mathrm{PH}$ is a major cause of morbidity and mortality in patients with hemoglobinopathies and chronic haemolytic anaemias including sickle cell disease (SCD) and thalassemia [479]. Mutations (G208C) in NFU1, a mitochondrial protein that is involved in the biosynthesis of iron-sulfur clusters develop PAH in 70\% of cases [480]. Iron accumulates in the lungs and AMs of individuals with $\mathrm{PH}$ [481]. Iron handling, in particular the hepcidin/FPN axis has been shown to be important in the vascular remodelling and endothelial cell dysfunction associated with PAH and loss of the iron regulatory protein Irp1 leads to the development of $\mathrm{PH}$ in mice [482-484]. While the role of iron in vascular endothelial and smooth muscle cells has been well studied; little is known regarding the role of iron in the perivascular immune cell infiltrates observed in $\mathrm{PH}$. Circulating macrophages recruited to the lung that contribute to pulmonary vascular remodelling in $\mathrm{PH}$ have a distinct iron and HO-1 rich phenotype driven by haemoglobin [485], however whether other immune cells also display alterations in iron handling remains to be determined.

Increases in both zinc and copper transport have also been implicated in pulmonary vascular homeostasis $[486,487]$ and selenium levels may be lower in individuals with PH [488]. Pulmonary hypertension can be triggered by chronic hypoxia. Hypoxia has been shown to cause an NO-dependent increase in labile zinc in mouse lung endothelial cells from isolated perfused lungs (IPL) [489]. Hypoxic restriction is attenuated in IPL from mice treated with a zinc chelator suggesting a role for chelatable zinc in modulating hypoxic pulmonary vasoconstriction. Furthermore, zinc homeostasis has been implicated in PH as disruption of the zinc importer ZIP12 (Slc39a12) gene attenuates the development of $\mathrm{PH}$ in rats housed in a hypoxic environment [486]. Increased expression of copper-related genes, copper uptake transporter (CTR1), copper efflux pump ATP7A, and lysyl oxidase (a copper-dependent enzyme), was observed in mice with hypoxia induced PAH. Increased expression of CTR1 has also been observed in response to hypoxia within macrophages [490]. However, administration of a low copper diet in experimental models of $\mathrm{PH}$ had no effect on right ventricular (RV) failure [491]. Dietary administration of selenium to broiler chickens prevented RV hypertrophy associated with PH syndrome [492] suggesting some possible benefit for $\mathrm{PH}$. Little is known regarding the role of these trace metals in immune cell function in the lung or interaction with the airway microbiome in $\mathrm{PAH}$.

\section{Mycobacterial lung infections: tuberculosis and non-tuberculous mycobacteria (NTM)}

Lung infections caused by mycobacteria cause a significant global health burden. Mycobacterium tuberculosis $(M t b)$, the causative agent of tuberculosis (TB), causes 9 million new infections each year and over 1 million deaths [493]. While $M t b$ is a human adapted pathogen, non-tuberculous mycobacteria (NTM) consist of over 200 species of environmental mycobacteria, some of which can opportunistically infect humans. NTM lung infections most often occur in those with underlying conditions including COPD, cystic fibrosis and bronchiectasis. NTM infections are less prevalent than TB but the prevalence of these infections is increasing globally [494]. This is concerning as the treatment for NTM requires multi-drug regimen lasting over a year with only a $50-80 \%$ success rate [495]. Furthermore, recurring infections after treatment completion are common.

\section{Mycobacteria and trace metal acquisition}

Pathogenic mycobacteria must be able to acquire nutrients to support growth, survival and persistence within the host [496]. These intracellular pathogens acquire 
iron by stealing it from their host through the production of siderophores as well as utilisation of host ironbinding proteins heme, haemoglobin and transferrin [497]. Siderophores are compounds produced by some microorganisms with a high-affinity for iron in response to iron deficient conditions [498]. There are three types of mycobacterial siderophores: mycobactin, carboxymycobactin and exochelin. Mycobactin is associated with the bacterial cell envelope and transports iron through the cell envelope into the cytoplasm. The structure of mycobactin varies between bacterial species. However, its general lipid-soluble structure allows the binding of one iron atom per molecule at a very high affinity [498, 499]. Carboxymycobactin has a very similar structure to mycobactin but it is modified by the addition of a carboxylic acid group, making it more hydrophilic, thus it is an extracellular siderophore that is secreted by the bacteria. Exochelins are extracellular siderophores and are produced only by fast growing mycobacteria. Their structure consists of ornithine-derived hydroxamates as iron coordination sites allowing them to acquire iron from insoluble sources such as ferric oxide and ferritin [499].

Mycobacteria also have alternative mechanisms to siderophores to obtain iron from their host [500]. Heme is one of the largest human iron stores and as a result it is often exploited by bacteria as a source of iron. M. tuberculosis is capable of importing heme and either extracting the iron if needed or storing the heme. M. tuberculosis also requires heme itself and encodes its own heme biosynthesis pathway, which is essential if an external heme source is not provided [501-503]. Mycobacteria can also use haemoglobin as an iron source to support their growth. The growth supported by haemoglobin was accompanied by an decrease in siderophore production, suggesting an alternative iron-acquisition system [504].

Pathogenic bacteria must be able to safely store intracellular iron once it has been acquired from the host and like vertebrates, they use ferritins and bacterioferritins (Bfrs) to do so. Typically, ferritins assemble into spherical particles or 'nanocages' consisting of 24 subunits with a ferroxidase centre within each subunit. $M$. tuberculosis has both ferritin and Bfrs (BfrA and BfrB). The expression of $b f r A$ and $b f r B$ genes is regulated by the iron sensitive regulator IdeR such that ferritins levels adapt to the level of iron [505]. Deletion of BfrA and BfrB from $M$. tuberculosis results in a decreased ability to withstand oxidative stress, antibiotic treatment and survival within human macrophages. Loss of both genes leads to reduced virulence in a guinea pig model of TB infection and loss of $b f r B$ lead to an inability for $M$. tuberculosis to persist in mice $[506,507]$. These studies show the iron storage system of $M$. tuberculosis to be an attractive drug target, as it not only is required for full virulence but also to withstand antibiotic treatment (including the frontline TB drug isoniazid). M. avium encodes only one ferritin homologue (BfrA) which is less important to iron homeostasis and virulence in $M$. tuberculosis. As $M$. avium most often causes infections in those with chronic lung diseases such as COPD, CF and non-CF bronchiectasis where iron levels in the lungs are increased, one could speculate that iron storage within M. avium is particularly crucial to persist in the host and thus we could speculate that the BfrA homologue in M. avium could be important for this opportunistic pathogen in dealing with abundant iron. Interestingly, the crystal structure of Bfr from M. smegmatis (which is $87 \%$ identical to its homologue in $M$. tuberculosis) revealed that it contained zinc in its di-nuclear metal binding site [508]. The biological implications of this and whether this is also true for the Bfrs in other mycobacteria have not yet been explored.

\section{$T B$ and the microbiome}

Studies have shown that $M$. tuberculosis infection affects both the lung and the gut microbiome of its host. However, the role of other mycobacterial species on the microbiome still needs to be elucidated. Interactions between the microbial communities of the gut and lung of an infected host can influence the outcome of $M$. tuberculosis disease progression and response to treatment [509]. The microbiota of sputum samples from TB patients were more diverse compared to respiratory secretions of healthy participants. Bacteria such as Pseudomonas and Cupriavidus were exclusive to TB patients and could influence the onset or development of infection [510]. BALF samples of TB patients showed a similar diversity in the lower respiratory tract with Cupriavidus bacteria possibly acting as a cofactor to secondary $\mathrm{TB}$ infections [511]. Furthermore, oral microbes present in the lungs of HIV patients on anti-retroviral therapy produce SCFAs, such as butyrate and propionate that increased patient susceptibility to $M$. tuberculosis infections by inhibiting the production of IFN-y and IL-17A cytokines [512].

Mice infected with M. tuberculosis by aerosol lost their gut microbial diversity and recovered with a different microbial composition [513]. Furthermore, the administration of first-line TB antibiotics almost immediately (within 1 week) altered the intestinal microbiota of $M$. tuberculosis infected mice with distinct and long lasting effects [514]. Faecal samples from clinical pulmonary TB patients had dysbiosis of their gut microbiota with a significant decrease in the SCFA-producing bacteria. A decrease in SCFA-producing bacteria have also been found in systemic inflammatory disease and therefore, loss of these bacteria in TB patients may indicate systemic inflammation and an impaired immune response 
[515]. There have been attempts to create a classification model using the abundance of gut microbes to discriminate between healthy and diseased patients [515].

\section{Trace metals, nutritional immunity and mycobacteria}

Elemental analysis of the phagosomes of peritoneal macrophages of C57BL/6 mice infected with M. avium, M. tuberculosis or M. smegmatis showed that the iron concentration of the phagosomes containing the pathogenic mycobacteria was significantly higher [83]. Radioactive iron-loaded transferrin was used to reveal that the infected macrophages acquire extracellular iron and delivers it to vacuoles containing $M$. avium through the transferrin receptor. However, when the macrophages were activated with IFN- $\gamma$ before infection, iron accumulation by the pathogenic mycobacterial phagosomes was prevented. IFN-y is known to downregulate the transferrin receptor in order to reduce the iron pool and enhance macrophage activity. Furthermore, when the macrophages were treated after infection, there was no change in iron concentration indicating macrophage anergy to IFN-y activation [83]. Iron is extracted from transferrin by mycobactins but holo-transferrin can also be taken up by M. tuberculosis within macrophages [516].

The Nramp1/Slc11a1 gene is associated with the controlling intracellular pathogens such as Mycobacteria, Salmonella and Leishmania. Several studies have reported polymorphisms in Nramp1 which are associated with increased susceptibility to tuberculosis and leprosy (caused by M. leprae) in humans [517-519]. NRAMP1 is a transmembrane protein localised to lysosomal membranes of phagocytic cells and is recruited to the phagosome upon infection [520-522]. It belongs to a family of divalent cation transporters and functional studies have shown that it transports iron out of the phagosome [523-526]. A recent report found that NRAMP1 restricts Salmonella growth in mice through magnesium deprivation, but whether this is via magnesium being directly transported by NRAMP1 has not yet been determined [527]. NRAMP1 is also known to have roles in nitric oxide production, phagosome maturation and induction of lipocalin 2 [528-530]. Despite studies in macrophages showing that NRAMP1 can restrict mycobacterial growth, deletion of Nramp1 in mice infected with $M$. tuberculosis did not result in increased bacterial burden [531]. This is in contrast to M. avium, where expression of the $\operatorname{Nrampl}^{\mathrm{D} 169}$ allele (which results in deficient function and/or expression) in mice results in these animals being highly susceptible to M. avium [532]. $M$. avium growth increased in a dose-dependent manner with iron administration in mice expressing the functional Nramp $1^{G 169}$ allele indicated that excess iron can impair or overcome the function of the NRAMP1 protein
[532]. Further studies have argued that NRAMP1 in fact transports iron into the phagosome upon its recruitment to aid mycobacterial killing be catalysing the Fenton/Haber-Weiss reaction to produce hydroxyl radicles [533]. Further investigation is required to fully elucidate the role of NRAMP1 during mycobacterial infections and whether the importance of its role differs depending on the mycobacterial species causing infection.

Murine BMDM infected with M. avium upregulated the transcription of heavy-chain $(\mathrm{H})$-ferritin in response to the stimulation of toll-like receptor-2. This could play a role in starving the mycobacterium of iron by storing iron atoms away from the pathogen while also driving macrophage polarisation towards a glycolytic M1 antibacterial phenotype via $\operatorname{HIF} 1 \alpha[534,535]$. Iron deficiency induced stabilisation of the transcription factor HIF- $1 \alpha$ promoting sustained IFN- $\gamma$-mediated polarization towards the M1 phenotype in vivo and the addition of the iron chelator deferoxamine to M. tuberculosis-infected human primary macrophages promoted the M1 phenotype in vitro $[535,536]$. LCN2 is involved in the innate immune response and can bind to the bacterial siderophore-iron complex, carboxymycobactin and restricts bacterial growth. In vitro studies showed that Lcn2 restricts the growth of $M$. avium in vitro while the mycobacteria also induced Lcn2 production by murine BMDMs [537]. Further in vivo studies with LCN2, myeloid differentiation primary response (MyD88) and TIR-domain-containing adapter-inducing interferon- $\beta$ (TRIF) knock out mice confirmed this observation [537]. LCN2 was elevated and reduced $M$. avium growth in the blood of infected mice. Since M. avium is an intracellular pathogen, subcellular imaging showed that the mycobacteria avoid LCN2-mediated immunity by residing in the Rab11 + endocytic recycling pathway which still allows them access to transferrin [537]. The Rab5 and Rab7 GTPases were also investigated as they control early and late endosomal fusion, respectively. A well-known strategy that pathogenic mycobacteria employ to persist within macrophages is by inhibiting phagosomal maturation. In vitro studies using murine BMDM revealed that those expressing the dominant negative Rab5, Rab5(S34N), limited the concentration of iron within the $M$. avium phagosome which resulted in the phagosome maturing and ultimately killing the mycobacteria when compared to wild-type Rab5 BMDM. Therefore, early endosomal fusion is required for the mycobacteria to persist in these early phagosomal compartments but $M$. avium requires an adequate iron supply to prevent further phagosome maturation [538].

Copper is essential to mycobacteria with its role as a required co-factor in the aa3-type cytochrome c oxidase, which is essential for M. tuberculosis growth. However, 
most studies on copper in mycobacteria have focused on resistance to copper stress as copper toxicity in the macrophage phagosome is a known antimycobacterial strategy employed by the host. Several studies have characterised the transcriptional response to copper but the exact mechanisms of copper uptake and not yet fully defined [539]. However, copper uptake is known to be mediated by outer membrane porins in mycobacteria [540]. Other membrane proteins have been identified, such as CtpV which acts as a copper efflux pump and MctB which regulates intracellular copper levels, as being important for $M$. tuberculosis copper resistance and virulence $[541,542]$. M. tuberculosis senses copper via two copper responsive transcriptional regulators CsoR and RicR [543, 544]. Both of these regulators control genes that contribute to copper resistance and virulence of $M$. tuberculosis. DNA binding of CsoR is affected by binding to copper and derepresses the expression of an operon which includes $\operatorname{ctp} V$, in response to high copper levels. RicR regulates a copper induced regulon including the methallothionein $m y m T$ a copper binding protein that helps to protect against copper toxicity [545]. Mutation of RicR resulting in an inability to respond to copper led to increased copper sensitivity and attenuated virulence in mice suggesting that this regulon is important for protecting $M$. tuberculosis against copper stress during infection [546]. The toxicity of copper in bacteria is thought to be due to copper removing iron from iron sulfur clusters, indeed, transcriptomic data from M. tuberculosis under copper stress also implicates damage of iron sulfur cluster enzymes [12, 539].

Zinc is an essential nutrient for mycobacteria and so access to this metal must be crucial for establishing and persisting in the host [547]. Mycobacteria can sense zinc levels and adapt to allow for persistence during zinc limited conditions [548-550]. The zinc import systems in mycobacteria are not clearly defined but are likely to consist of unspecific transport divalent cations transporters from the CorA, MgtE, ZIP, NiCo and Pit families of uptake systems. P-type ATPases may also be able to import zinc ions into the cell [551]. Export of zinc to maintain zinc homeostasis during exposure to high zinc levels is an important resistance mechanism as M. tuberculosis infected macrophages import high amounts of zinc into the phagosome. $M$. tuberculosis increases the expression of a P-type ATPase CtpC in response to infection and exposure to zinc. Loss of this ATPase leads to increased zinc levels within the bacteria and a reduced capacity to grow within macrophages [79]. Another gene Rv3929, which is encoded in the same operon as CtpC, is upregulated in response to zinc stress. This gene encodes a putative metallochaperone that could potentially supply zinc to the $\mathrm{CtpC}$ transporter. Zinc sequestration may also occur to restrict the growth of extracellular bacteria present in the necrotic granulomas in active TB as S100 proteins are produced at high levels by neutrophils in the granulomas of TB patients[552]. Zinc starvation has been shown to lead to ribosome hibernation in M. smegmatis [553]. This ribosome hibernation results in antibiotic tolerance of $M$. tuberculosis in mouse lungs and so while zinc sequestration may restrict the growth of the bacteria it may also potentially cause the bacteria to be more difficult to treat and eradicate during infection [554].

Manganese, nickel and cobalt are also essential nutrients for M. tuberculosis. CtpC was also found to transport manganese allowing for metalation of secreted proteins, and deletion of $\mathrm{CtpC}$ resulted in altered manganese homeostasis and increased sensitivity to oxidative stress [555]. Nickel is used as a co-factor for the M. tuberculosis urease UreA [556, 557]. Mycobacteria are some of the few bacteria that can synthesize vitamin $B_{12}$, a complex cobalt containing molecule, and have three vitamin $\mathrm{B}_{12}$-dependent enzymes [558-560]. However, the mechanisms by which these metals are taken up by mycobacteria not yet known. Host sequestration of manganese (and zinc) by S100 proteins, including calprotectin, a chelator of extracellular iron and zinc, is induced in response to bacterial infections [561]. Sequestration of extracellular manganese and zinc is likely to occur via S100 proteins as they are highly produced by neutrophils in the lung granulomas in TB patients [552]. This would likely impact the growth of extracellular bacteria that are present within necrotic granulomas in active TB, but further studies are required to clearly determine the role of these metals in M. tuberculosis virulence.

\section{Therapy}

An alteration in metal homeostasis, either systemically or within the lung compartment is associated with the pathogenesis and poor outcomes in several respiratory diseases. Therefore, correcting metal dysregulation could provide a novel approach to treat these diseases, potential counteracting one of the driving forces in the pathogenesis of these diseases. The potential of using iron chelators to reduce pulmonary iron overload in chronic lung diseases such as COPD, CF and IPF are an exciting prospect $[319,562-564]$. Reducing iron overload specifically in the lung in chronic lung diseases could help to counteract the metal dysregulation driving chronic inflammation and also potentially limit the respiratory infections that commonly occur in people living with these conditions by depriving airway microbes from this essential nutrient. However, as iron deficiency and anaemia is prevalent in the majority of these diseases, any direct targeting of iron in the lung must avoid reducing systemic iron levels further and re-supplementing systemic iron in iron deficient 


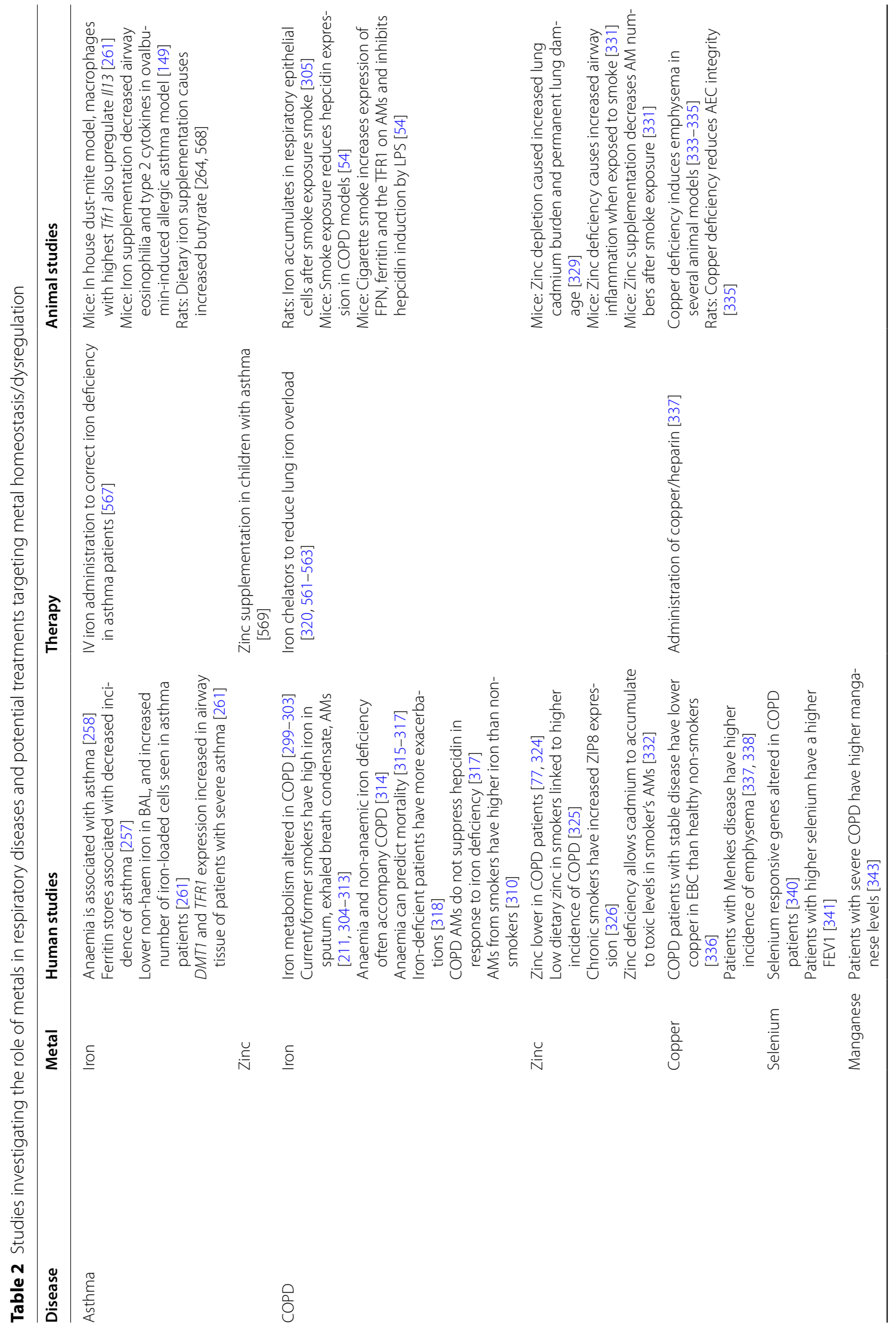


Healy et al. Respir Res ～(2021) 22:133

Page 29 of 44

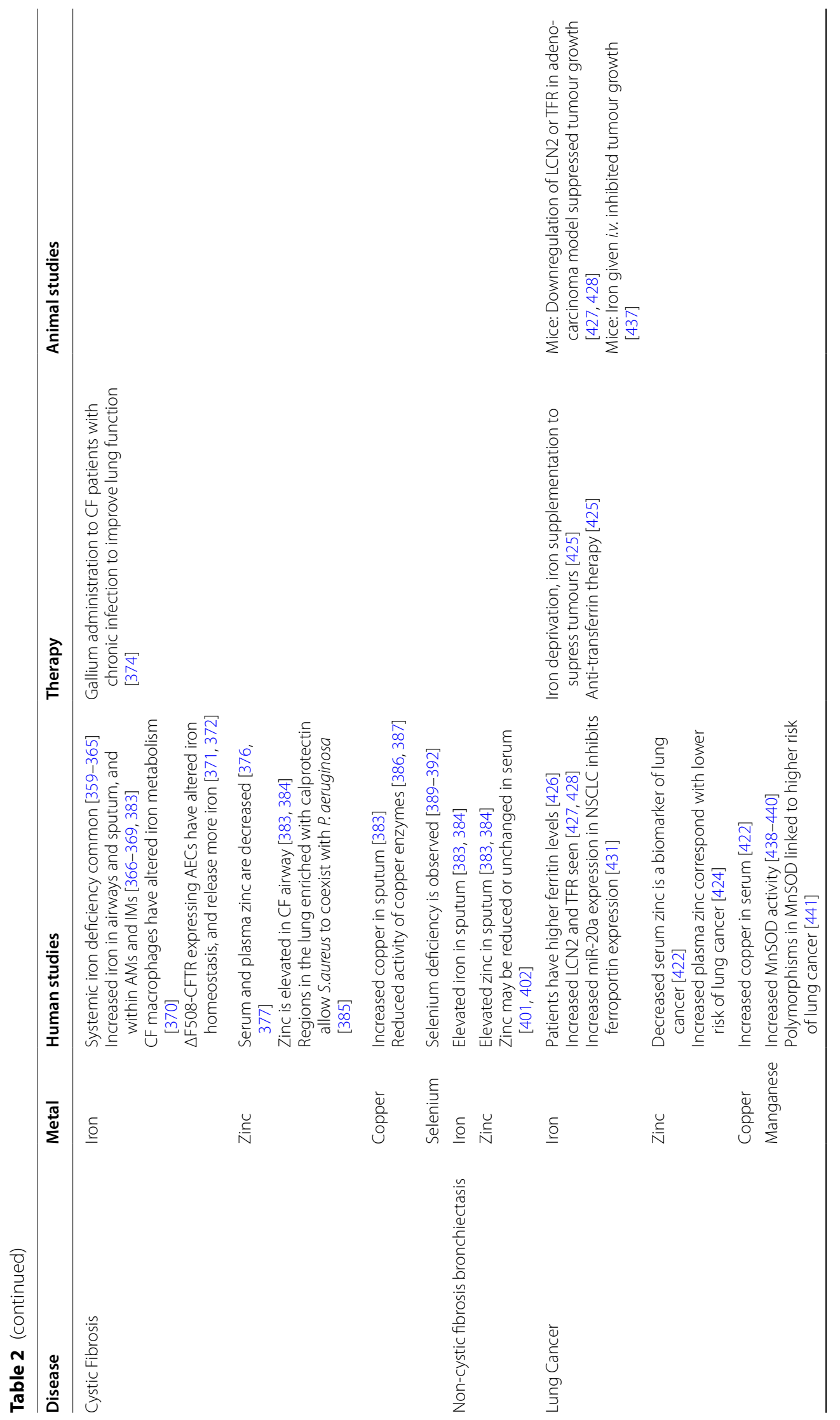


Healy et al. Respir Res ～(2021) 22:133

Page 30 of 44

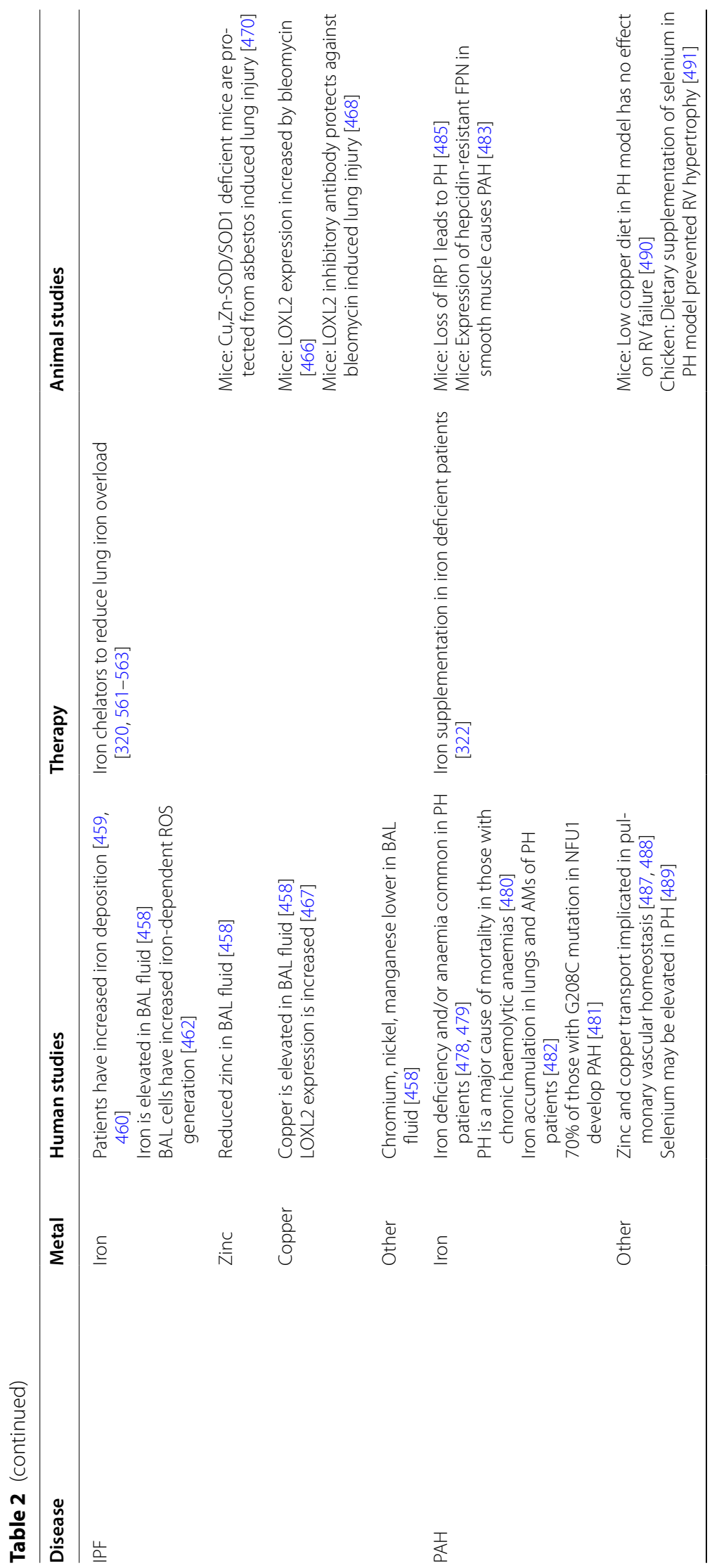




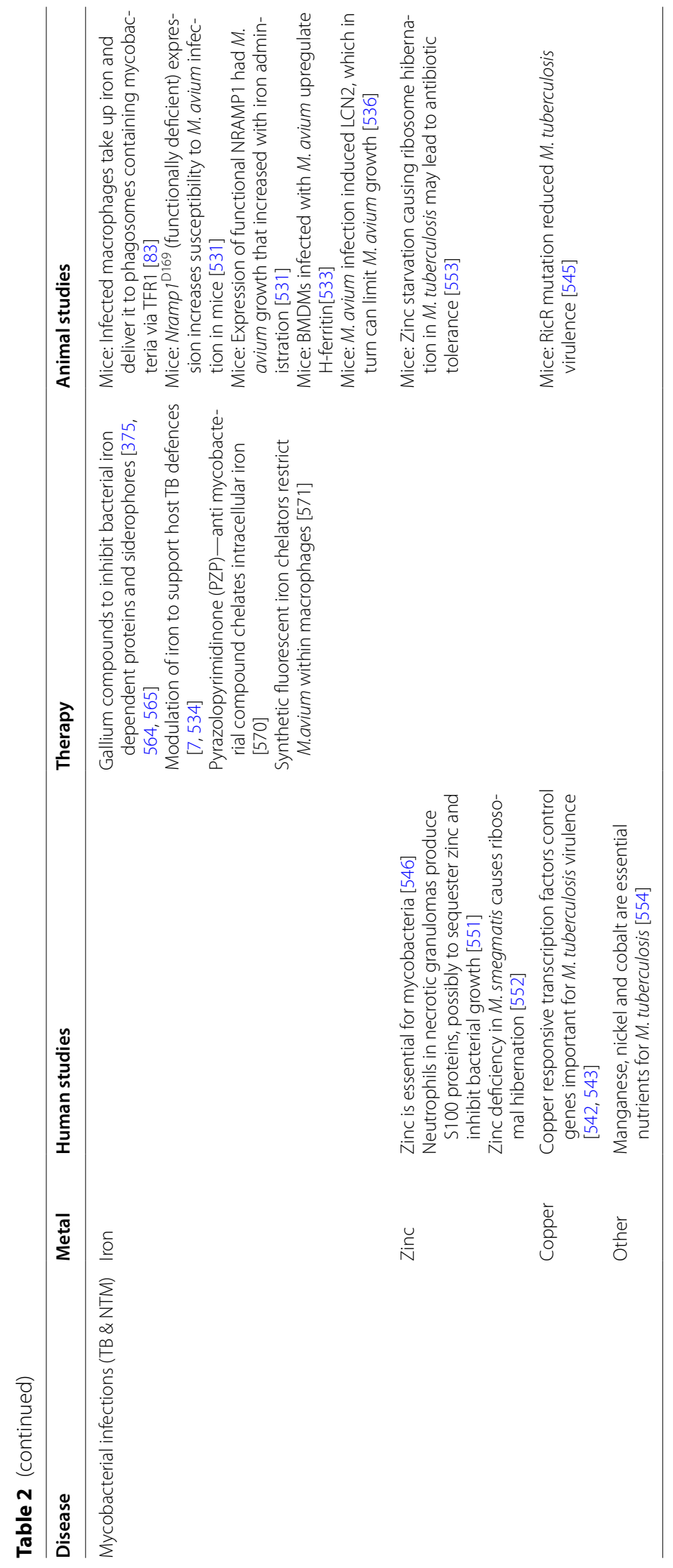


and anaemic patients may benefit overall survival and exercise capacity. Another approach to counteracting high iron in the lung is the use of gallium, a non-essential metal that acts as an iron mimetic. Gallium containing compounds have been shown to improve the lung function of CF patients with chronic infections. Gallium compounds have been shown to inhibit bacterial iron dependent processes in major respiratory pathogens, $P$. aeruginosa and $M$. tuberculosis, $M$ avium, and $M$. abscessus $[373,374,565,566]$. As gallium can replace iron in iron dependent bacterial proteins but is not redox active it interferes with function of iron-dependent essential processes such as the respiratory chain and kills the bacteria. Metal metabolism dysfunction could also potentially be corrected very easily by dietary supplementation, such as selenium supplementation for influenza and zinc supplementation for asthma. However, these treatments are likely to only have a beneficial effect in patients with deficiencies in these metals and so personalised treatment approaches are vital.

Immunonutrition is a novel concept where patients are given a personalized diet rich in vitamins and minerals such as zinc, selenium and iron to modulate immune responses in order to improve disease outcome and recovery. The modulation of the gut microbiota by immunonutrition has been reviewed as a potential therapeutic strategy for obese COVID-19 patients [567]. This novel idea of utilizing the gut-lung axis might also have potential influences on the outcome of bacterial infections such as $M$. tuberculosis that rely on metals such as iron for their replication and growth. Perhaps a personalized diet to deprive the bacteria of certain metals may one day be a potential therapeutic strategy for pulmonary bacterial infections. An overview of potential therapies for respiratory disease by targeting metal homeostasis and dysregulation are presented in Table 2.

\section{Conclusion}

The lungs are a unique compartment in the body in that they are constantly exposed to the environment. We are constantly breathing in air from our surroundings which consists of microorganisms, particles, chemicals, and pollutants. The resident immune cells within this niche must be able to deal with the influx of microbes and particles without causing inflammation. Unfortunately, environmental, lifestyle and genetic factors can lead to chronic lung inflammation resulting in an array of lung diseases that result in obstruction and damage to the airways and a decline in lung function. Furthermore, recurrent respiratory infections are common among those with chronic lung disease and contribute to progressive decline in lung function. The role of metabolites and nutrients present in lung microenvironment are just beginning to be investigated and appreciated. Metals essential to most organisms, such as iron, copper and zinc are found to be altered in several lung diseases. These metals play important roles both in the function of immune cells in the lung and in the microbes present in the airways. There remains much to be explored on the cause and source of this metal dysregulation during lung disease, as well as the exact mechanistic consequences of this on the immune cells and the constituents of the airway microbiota. We believe that targeting disturbed metal metabolism seen in many lung diseases is a novel approach that could potentially address both immune-driven pathology and the increased occurrence of respiratory infections. We hope that future work will further our understanding of the complex interactions between lung immunity, the lung microbiome and the lung microenvironment and will lead to much improved treatments for the many diseases of the lung that yet remain to be curable.

\section{Abbreviations}

2DG: 2-Deoxy-D-glucose; AD: Adenocarcinoma; AEC: Alveolar epithelial cells; ALAS: 5-Aminoevulinate synthase; AM: Alveolar macrophage; ATP: Adenosine triphosphate; BALF: Bronchoalveolar lavage fluid; BCR: B cell receptor; Bfr: Bacterioferritin; BMDM: Bone marrow derived macrophage; CDCs: Conventional dendritic cells; CF: Cystic fibrosis; CFTR: CF transmembrane conductance regulator; COPD: Chronic obstructive pulmonary disease; CXCR: Chemokine receptor; DAMP: Damage associated molecular patterns; DCs: Dendritic cells; DMT1: Divalent metal transporter 1; EBC: Exhaled breath condensate; EPX: Eosinophil peroxidase; FEV1: Forced expiratory volume in 1s; FPN: Ferroportin; FTH: Ferritin heavy chain; GM-CSF: Granulocyte macrophage-colony stimulating factor; Gpx4: Glutathione peroxidase 4; HFE: Hereditary hemochromatosis (HH) susceptibility gene; HIF-1a: Hypoxia inducible factor-1a; HO-1: Heme oxygenase 1; iBALT: Bronchial associated lymphoid tissue; IFN-y: Interferon-y; ILCs: Innate leukocyte cells; IM: Interstitial macrophage; iNKT: Invariant natural killer cells; IPF: Idiopathic pulmonary fibrosis; IPL: Isolated perfused lungs; IRF: Interferon regulatory factor; iTAMs: Iron-loaded TAMs; JNK: C-Jun N-terminal kinase; KIR: Killer cell-immunoglobulin like receptor; LCN-2: Lipocalin-2; LFA: Lymphocyte function-associated antigen; LfR: Lactoferrin receptor; LIAS: Lipoic acid synthase; LOXL2: Lysyl oxidase-like 2; LPS: Lipopolysaccharide; LRP1: Lipoprotein receptor related protein 1; LTi: Lymphoid tissue inducer; MAIT: MR1-restricted T cells; MHC: Major histocompatibility complex; MnSOD: Manganese superoxide dismutase; moDCs: Monocyte-derived dendritic cells; MPO: Myeloperoxidase; mRNA: Messenger ribonucleic acid; MsrB1: Methionine sulfoxide reductase B1; MTs: Metallothioneins; MyD88: Myeloid differentiation primary response; NETs: Neutrophil extracellular traps; NK: Natural killer; NO: Nitric oxide; NRAMP1: Natural resistance associated macrophage protein 1; NTM: Non-tuberculous mycobacteria; $\mathrm{OH} \cdot:$ Hydroxyl radical; OX PHOS: Oxidative phosphorylation; PAH: Pulmonary arterial hypertension; pDCs: Plasmacytoid DCs; PDH: Pyruvate dehydrogenase; PH: Pulmonary hypertension; PHDs: Prolyl hydroxylases; RNSO: S-nitrosothiols; ROS: Reactive oxygen species; SC: Squamous cell; SCD: Sickle cell disease; SCFA: Short chain fatty acid; SCLC: Small cell lung cancer; SelK: Selenoprotein K; SOD: Superoxide dismutase; STAT: Signal transducer and activator of transcription; TAMs: Tumour associated macrophages; TB: Tuberculosis; TCR: T cell receptor; TGF: Transforming growth factor; Th: Helper T cell; TLR: Toll like receptor; TLS: Tertiary lymphoid structures; TME: Tumour microenvironment; Tregs: T regulatory cells; TFR1:Transferrin receptor 1; TRIF: TIR-domain-containing adapter-inducing interferon- $\beta ; T_{R M}$ : Lung resident memory T cells; tRNA: Transfer ribonucleic acid; aKGDH: a-Ketoglurate dehy-

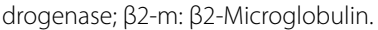




\section{Acknowledgements}

S.M.C, C.H., J.S., L.F., N.W. are supported by Science Foundation Ireland (SFI) Future Research Leaders Grant FRL4862. NMW is supported by Health Research Board Grant EIA-2019-004.

\section{Authors' contributions}

Conception: SMC; Preparation of manuscript: CH, NMW, SK, NCW, JS, LF, SMC; Manuscript revision and editing: SMC, CH, NMW, SCD; Figure preparation: JS; Table preparation: LF, NCW, CH. All authors read and approved the final manuscript.

\section{Funding}

S.M.C, C.H., J.S., L.F., N.W. are supported by Science Foundation Ireland (SFI) Future Research Leaders grant FRL4862. NMW is supported by Health Research Board Grant EIA-2019-004.

\section{Availability of data and materials}

Not applicable.

\section{Declarations}

\section{Ethics approval and consent to participate} Not applicable.

\section{Consent for Publication}

Not applicable.

\section{Competing Interests}

The authors have no competing interests to declare.

\section{Author details}

${ }^{1}$ School of Medicine, Trinity Biomedical Sciences Institute, Trinity College Dublin, Dublin, Ireland. ${ }^{2}$ Tallaght University Hospital, Dublin, Ireland. ${ }^{3}$ Joan and Sandford I. Weill Department of Medicine, Division of Pulmonary and Critical Care Medicine, Weill Cornell Medicine, New York City, USA.

Received: 24 February 2021 Accepted: 15 April 2021

Published online: 29 April 2021

\section{References}

1. Organisation, W.H. The top 10 causes of death. 2020; https://www.who. int/news-room/fact-sheets/detail/the-top-10-causes-of-death.

2. Palmer LD, Skaar EP. Transition Metals and Virulence in Bacteria. Annu Rev Genet. 2016:50:67-91.

3. Cornelis P, Dingemans J. Pseudomonas aeruginosa adapts its iron uptake strategies in function of the type of infections. Front Cell Infect Microbiol. 2013;3:75.

4. Bachman MA, et al. Klebsiella pneumoniae yersiniabactin promotes respiratory tract infection through evasion of lipocalin 2. Infect Immun. 2011;79(8):3309-16.

5. De Voss JJ, et al. The salicylate-derived mycobactin siderophores of Mycobacterium tuberculosis are essential for growth in macrophages. Proc Natl Acad Sci U S A. 2000;97(3):1252-7.

6. Pidcock KA, et al. Iron acquisition by Haemophilus influenzae. Infect Immun. 1988:56(4):721-5.

7. Phelan JJ, et al. Modulating Iron for Metabolic Support of TB Host Defense. Front Immunol. 2018; 9(2296).

8. Smith DJ, et al. Targeting iron uptake to control Pseudomonas aeruginosa infections in cystic fibrosis. Eur Respir J. 2013;42(6):1723-36.

9. Lonergan ZR, Skaar EP. Nutrient zinc at the host-pathogen interface. Trends Biochem Sci. 2019;44(12):1041-56.

10. Babu U, Failla ML. Respiratory burst and candidacidal activity of peritoneal macrophages are impaired in copper-deficient rats. J Nutr. 1990;120(12):1692-9.

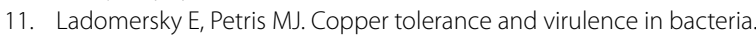
Metallomics. 2015:7(6):957-64.
12. Macomber L, Imlay JA. The iron-sulfur clusters of dehydratases are primary intracellular targets of copper toxicity. Proc Natl Acad Sci U S A. 2009;106(20):8344-9.

13. Urban CF, et al. Neutrophil extracellular traps contain calprotectin, a cytosolic protein complex involved in host defense against Candida albicans. PLoS Pathog. 2009;5(10):e1000639.

14. Guillin $\mathrm{OM}$, et al. Selenium, selenoproteins and viral infection. Nutrients, 2019; 11(9).

15. O'Neill LA, Kishton RJ, Rathmell J. A guide to immunometabolism for immunologists. Nat Rev Immunol. 2016;16(9):553-65.

16. Metchnikoff E. Leçons sur la pathologie comparée de I"inflammation faites àl"Institut Pasteur en avril et ma. 1892.

17. O'Beirne SL, et al. Alveolar macrophage immunometabolism and lung function impairment in smoking and chronic obstructive pulmonary disease. Am J Respir Crit Care Med. 2020;201(6):735-9.

18. Fricker M, Gibson PG. Macrophage dysfunction in the pathogenesis and treatment of asthma. Eur Respir J. 2017;50(3):1700196.

19. Bruscia EM, Bonfield TL. Cystic fibrosis lung immunity: the role of the macrophage. J Innate Immun. 2016;8(6):550-63.

20. Ardain A, Marakalala MJ, Leslie A. Tissue-resident innate immunity in the lung. Immunology. 2020;159(3):245-56.

21. Guilliams $M$, et al. Alveolar macrophages develop from fetal monocytes that differentiate into long-lived cells in the first week of life via GM-CSF. J Exp Med. 2013;210(10):1977-92.

22. van de Laar $L$, et al. Yolk sac macrophages, fetal liver, and adult monocytes can colonize an empty niche and develop into functional tissue-resident macrophages. Immunity. 2016:44(4):755-68.

23. Snelgrove RJ, et al. A critical function for CD200 in lung immune homeostasis and the severity of influenza infection. Nat Immunol. 2008;9(9):1074-83.

24. Byrne AJ, et al. Pulmonary macrophages: key players in the innate defence of the airways. Thorax. 2015;70(12):1189-96.

25. Neupane AS, et al. Patrolling alveolar macrophages conceal bacteria from the immune system to maintain homeostasis. Cell. 2020;183(1):110-125.e11.

26. Bourdonnay E, et al. Transcellular delivery of vesicular SOCS proteins from macrophages to epithelial cells blunts inflammatory signaling. J Exp Med. 2015;212(5):729-42

27. Levine AM, et al. GM-CSF-deficient mice are susceptible to pulmonary group B streptococcal infection. J Clin Investig. 1999;103(4):563-9.

28. Gonzalez-Juarrero M, et al. Disruption of granulocyte macrophagecolony stimulating factor production in the lungs severely affects the ability of mice to control Mycobacterium tuberculosis infection. J Leukoc Biol. 2005;77(6):914-22.

29. Schneider $C$, et al. Alveolar macrophages are essential for protection from respiratory failure and associated morbidity following influenza virus infection. PLoS Pathog. 2014;10(4):e1004053.

30. $\mathrm{Yu} X$, et al. The cytokine TGF- $\beta$ promotes the development and homeostasis of alveolar macrophages. Immunity. 2017;47(5):903-912.e4.

31. Puttur F, Gregory LG, Lloyd CM. Airway macrophages as the guardians of tissue repair in the lung. Immunol Cell Biol. 2019;97(3):246-57.

32. Gibbings SL, et al. Three unique interstitial macrophages in the murine lung at steady state. Am J Respir Cell Mol Biol. 2017;57(1):66-76.

33. Schyns J, et al. Non-classical tissue monocytes and two functionally distinct populations of interstitial macrophages populate the mouse lung. Nat Commun. 2019;10(1):3964.

34. Chakarov S, et al. Two distinct interstitial macrophage populations coexist across tissues in specific subtissular niches. Science. 2019:363(6432):eaau0964.

35. Janssen WJ, et al. Fas determines differential fates of resident and recruited macrophages during resolution of acute lung injury. Am J Respir Crit Care Med. 2011;184(5):547-60.

36. Mould KJ, et al. Cell origin dictates programming of resident versus recruited macrophages during acute lung injury. Am J Respir Cell Mol Biol. 2017:57(3):294-306.

37. Machiels B, et al. A gammaherpesvirus provides protection against allergic asthma by inducing the replacement of resident alveolar macrophages with regulatory monocytes. Nat Immunol. 2017;18(12):1310-20. 
38. Byrne AJ, et al. Dynamics of human monocytes and airway macrophages during healthy aging and after transplant. J Exp Med, 2020; 217(3).

39. Guilliams M, et al. Establishment and maintenance of the macrophage niche. Immunity. 2020;52(3):434-51.

40. Winn NC, Volk KM, Hasty AH. Regulation of tissue iron homeostasis: the macrophage "ferrostat". JCI Insight, 2020; 5(2).

41. Roth-Walter F, et al. Linking iron-deficiency with allergy: role of molecular allergens and the microbiome. Metallomics. 2017;9(12):1676-92.

42. Ghio AJ, et al. DMT1 expression is increased in the lungs of hypotransferrinemic mice. Am J Physiol-Lung Cell Mol Physiol. 2003;284(6):L938-44.

43. Wygrecka M, et al. Shedding of low-density lipoprotein receptorrelated protein-1 in acute respiratory distress syndrome. Am J Respir Crit Care Med. 2011;184(4):438-48.

44. Beker Aydemir T, et al. Zinc transporter ZIP14 functions in hepatic zinc, iron and glucose homeostasis during the innate immune response (Endotoxemia). PLoS ONE. 2012;7(10):e48679.

45. Pisu D, et al. Dual RNA-Seq of Mtb-infected macrophages in vivo reveals ontologically distinct host-pathogen interactions. Cell Rep. 2020;30(2):335-350.e4

46. Wesselius LJ, Flowers $\mathrm{CH}$, Skikne BS. Alveolar macrophage content of isoferritins and transferrin: comparison of nonsmokers and smokers with and without chronic airflow obstruction. Am Rev Respir Dis. 1992;145(2_pt_1):311-6.

47. Yang F, et al. Apical location of ferroportin 1 in airway epithelia and its role in iron detoxification in the lung. Am J Physiol Lung Cell Mol Physiol. 2005;289(1):L14-23.

48. Neves J, et al. Disruption of the hepcidin/ferroportin regulatory system causes pulmonary iron overload and restrictive lung disease. EBioMedicine. 2017:20:230-9.

49. Lim D, et al. The hepcidin-ferroportin axis controls the iron content of Salmonella-containing vacuoles in macrophages. Nat Commun. 2018;9(1):2091.

50. Willemetz A, et al. Iron-and hepcidin-independent downregulation of the iron exporter ferroportin in macrophages during Salmonella infection. Front Immunol. 2017:8:498

51. Babitt $J$, et al. Bone morphogenetic protein signaling by hemojuvelin regulates hepcidin expression. Nat Genet. 2006;38(5):531-9.

52. Nemeth $\mathrm{E}$, et al. IL-6 mediates hypoferremia of inflammation by inducing the synthesis of the iron regulatory hormone hepcidin. J Clin Investig. 2004;113(9):1271-6.

53. Nguyen NB, et al. Hepcidin expression and iron transport in alveolar macrophages. Am J Physiol Lung Cell Mol Physiol. 2006;291(3):L417-25.

54. Perez $\mathrm{E}$, et al. Hepcidin is essential for alveolar macrophage function and is disrupted by smoke in a murine chronic obstructive pulmonary disease model. J Immunol. 2020;205(9):2489-98.

55. Brown BL, et al. Structure of the mitochondrial aminolevulinic acid synthase, a key heme biosynthetic enzyme. Structure. 2018;26(4):580-9.

56. Schmitt TH, Frezzatti W, Schreier S. Hemin-induced lipid membrane disorder and increased permeability: a molecular model for the mechanism of cell lysis. Arch Biochem Biophys. 1993;307(1):96-103.

57. Knutson MD, et al. Iron release from macrophages after erythrophagocytosis is up-regulated by ferroportin 1 overexpression and downregulated by hepcidin. Proc Natl Acad Sci USA. 2005;102(5):1324-8.

58. van den Heuvel MM, et al. Regulation of CD163 on human macrophages: cross-linking of CD163 induces signaling and activation. J Leukoc Biol. 1999;66(5):858-66.

59. Simões RL, et al. Proinflammatory responses of heme in alveolar macrophages: repercussion in lung hemorrhagic episodes. Mediators Inflamm. 2013;2013:946878.

60. Stocker $\mathrm{R}$, et al. Bilirubin is an antioxidant of possible physiological importance. Science. 1987;235(4792):1043-6

61. Vitali SH, et al. Heme oxygenase-1 dampens the macrophage sterile inflammasome response and regulates its components in the hypoxic lung. Am J Physiol Lung Cell Mol Physiol. 2020:318(1):L125-I134.

62. Oberle $\mathrm{S}$, et al. The antioxidant defense protein ferritin is a novel and specific target for pentaerithrityl tetranitrate in endothelial cells. Biochem Biophys Res Commun. 1999;261(1):28-34.

63. Flo TH, et al. Lipocalin 2 mediates an innate immune response to bacterial infection by sequestrating iron. Nature. 2004;432(7019):917-21.
64. Agoro R, Mura C. Inflammation-induced up-regulation of hepcidin and down-regulation of ferroportin transcription are dependent on macrophage polarization. Blood Cells Mol Dis. 2016;61:16-25.

65. Poss KD, Tonegawa S. Heme oxygenase 1 is required for mammalian iron reutilization. Proc Natl Acad Sci. 1997:94(20):10919-24.

66. Agoro R, et al. Cell iron status influences macrophage polarization. PLoS ONE. 2018;13(5):e0196921.

67. Sindrilaru A, et al. An unrestrained proinflammatory M1 macrophage population induced by iron impairs wound healing in humans and mice. J Clin Investig. 2011;121(3):985-97.

68. Artyomov MN, Sergushichev A, Schilling JD. Integrating immunometabolism and macrophage diversity. Semin Immunol. 2016;28(5):417-24.

69. Russell DG, Huang L, Vanderven BC. Immunometabolism at the interface between macrophages and pathogens. Nat Rev Immunol. 2019:19(5):291-304

70. Pain D, Dancis A. Roles of Fe-S proteins: from cofactor synthesis to iron homeostasis to protein synthesis. Curr Opin Genet Dev. 2016:38:45-51.

71. Canal F, et al. Regulation of the cysteine desulfurase Nfs 1 and the scaffold protein IscU in macrophages stimulated with interferon- $\gamma$ and lipopolysaccharide. Arch Biochem Biophys. 2007;465(1):282-92.

72. Drapier JC, Hibbs JB Jr. Differentiation of murine macrophages to express nonspecific cytotoxicity for tumor cells results in L-argininedependent inhibition of mitochondrial iron-sulfur enzymes in the macrophage effector cells. J Immunol. 1988;140(8):2829-38.

73. Pereira $M$, et al. Acute iron deprivation reprograms human macrophage metabolism and reduces inflammation in vivo. Cell Rep. 2019;28(2):498511.e5.

74. Salvin SB, et al. The effect of dietary zinc and prothymosin alpha on cellular immune responses of RF/J mice. Clin Immunol Immunopathol. 1987:43(3):281-8

75. Nowak JE, et al. Prophylactic zinc supplementation reduces bacterial load and improves survival in a murine model of sepsis. Pediatr Crit Care Med. 2012;13(5):e323-9.

76. Wirth JJ, Fraker PJ, Kierszenbaum F. Zinc requirement for macrophage function: effect of zinc deficiency on uptake and killing of a protozoan parasite. Immunology. 1989;68(1):114-9.

77. Hamon R, et al. Zinc and zinc transporters in macrophages and their roles in efferocytosis in COPD. PLoS ONE. 2014;9(10):e110056.

78. Gao H, et al. Metal transporter Slc39a10 regulates susceptibility to inflammatory stimuli by controlling macrophage survival. Proc Natl Acad Sci U S A. 2017:114(49):12940-5.

79. Botella $\mathrm{H}$, et al. Mycobacterial p(1)-type ATPases mediate resistance to zinc poisoning in human macrophages. Cell Host Microbe. 2011;10(3):248-59.

80. Neyrolles O, Mintz E, Catty P. Zinc and copper toxicity in host defense against pathogens: Mycobacterium tuberculosis as a model example of an emerging paradigm. Front Cell Infect Microbiol. 2013;3:89.

81. Jones DG, Suttle NF. The effect of copper deficiency on the resistance of mice to infection with Pasteurella haemolytica. J Comp Pathol. 1983:93(1):143-9.

82. Crocker A, et al. Interaction of nutrition and infection: effect of copper deficiency on resistance to Trypanosoma lewisi. J Natl Med Assoc. 1992;84(8):697-706

83. Wagner D, et al. Elemental analysis of Mycobacterium avium-, Mycobacterium tuberculosis-, and Mycobacterium smegmatis-containing phagosomes indicates pathogen-induced microenvironments within the host cell's endosomal system. J Immunol. 2005;174(3):1491-500.

84. White $\mathrm{C}$, et al. A role for the ATP7A copper-transporting ATPase in macrophage bactericidal activity. J Biol Chem. 2009;284(49):33949-56.

85. Johnson MD, et al. Role of copper efflux in pneumococcal pathogenesis and resistance to macrophage-mediated immune clearance. Infect Immun. 2015;83(4):1684-94

86. Shen $\mathrm{Q}$, et al. Macrophage activation by IFN- $\gamma$ triggers restriction of phagosomal copper from intracellular pathogens. PLoS Pathog. 2018:14(11):e1007444

87. Jabado $\mathrm{N}$, et al. Natural resistance to intracellular infections: natural resistance-associated macrophage protein 1 (Nramp1) functions as a $\mathrm{pH}$-dependent manganese transporter at the phagosomal membrane. J Exp Med. 2000;192(9):1237-48. 
88. Davidsson L, et al. Identification of transferrin as the major plasma carrier protein for manganese introduced orally or intravenously or after in vitro addition in the rat. J Nutr. 1989;119(10):1461-4.

89. Lee BC, et al. Selenoprotein MsrB1 promotes anti-inflammatory cytokine gene expression in macrophages and controls immune response in vivo. Sci Rep. 2017;7(1):5119-5119.

90. Nelson SM, Lei X, Prabhu KS. Selenium levels affect the IL-4-induced expression of alternative activation markers in murine macrophages. J Nutr. 2011;141(9):1754-61.

91. Carlson BA, et al. Role of selenium-containing proteins in T-cell and macrophage function. Proc Nutr Soc. 2010;69(3):300-10.

92. Kopf M, Schneider C, Nobs SP. The development and function of lung-resident macrophages and dendritic cells. Nat Immunol. 2015;16(1):36-44.

93. Naik SH, et al. Development of plasmacytoid and conventional dendritic cell subtypes from single precursor cells derived in vitro and in vivo. Nat Immunol. 2007;8(11):1217-26.

94. Guilliams M, Lambrecht BN, Hammad H. Division of labor between lung dendritic cells and macrophages in the defense against pulmonary infections. Mucosal Immunol. 2013;6(3):464-73.

95. Sichien D, et al. Development of conventional dendritic cells: from common bone marrow progenitors to multiple subsets in peripheral tissues. Mucosal Immunol. 2017;10(4):831-44.

96. Hoffmann FM, et al. Distribution and Interaction of Murine Pulmonary Phagocytes in the Naive and Allergic Lung. Front Immunol. 2018. 9.

97. Cella M, et al. Plasmacytoid monocytes migrate to inflamed lymph nodes and produce large amounts of type I interferon. Nat Med. 1999:5(8):919-23.

98. Van Pottelberge GR, et al. Plasmacytoid dendritic cells in pulmonary lymphoid follicles of patients with COPD. Eur Respir J. 2010;36(4):781-91.

99. De Heer $\mathrm{HJ}$, et al. Essential role of lung plasmacytoid dendritic cells in preventing asthmatic reactions to harmless inhaled antigen. J Exp Med. 2004;200(1):89-98.

100. Verna $G$, et al. Iron overload mimicking conditions skews bone marrow dendritic cells differentiation into $\mathrm{MHCII}(\mathrm{low}) \mathrm{CD} 11 \mathrm{c}(+) \mathrm{CD} 11 \mathrm{~b}(+)$ F4/80(+) cells. Int J Mol Sci. 2020; 21(4)

101. Kramer $J$ L, et al. Differentiation of functional dendritic cells and macrophages from human peripheral blood monocyte precursors is dependent on expression of p21 (WAF1/CIP1) and requires iron. $\mathrm{Br} J$ Haematol. 2002:117(3):727-34.

102. Kitamura $\mathrm{H}$, et al. Toll-like receptor-mediated regulation of zinc homeostasis influences dendritic cell function. Nat Immunol. 2006;7(9):971-7.

103. Jenne $\mathrm{CN}$, et al. Neutrophils recruited to sites of infection protect from virus challenge by releasing neutrophil extracellular traps. Cell Host Microbe. 2013;13(2):169-80.

104. Kumawat K, et al. LAIR-1 limits neutrophilic airway inflammation. Front Immunol. 2019;10:842.

105. Casulli J, et al. CD200R deletion promotes a neutrophil niche for Francisella tularensis and increases infectious burden and mortality. Nat Commun. 2019;10(1):1-9.

106. Griffiths JS, et al. Differential susceptibility of Dectin-1 isoforms to functional inactivation by neutrophil and fungal proteases. FASEB J. 2018;32(6):3385-97.

107. Giacalone VD, et al. Neutrophil adaptations upon recruitment to the lung: new concepts and implications for homeostasis and disease. Int J Mol Sci. 2020; 21(3)

108. Kuebler W, et al. Contribution of selectins to leucocyte sequestration in pulmonary microvessels by intravital microscopy in rabbits. J Physiol. 1997:501(Pt 2):375.

109. Devi S, et al. Neutrophil mobilization via plerixafor-mediated CXCR4 inhibition arises from lung demargination and blockade of neutrophil homing to the bone marrow. J Exp Med. 2013;210(11):2321-36.

110. Ward PP, et al. Stimulus-dependent impairment of the neutrophil oxidative burst response in lactoferrin-deficient mice. Am J Pathol. 2008;172(4):1019-29.

111. Francis $\mathrm{N}$, et al. Lactoferrin inhibits neutrophil apoptosis via blockade of proximal apoptotic signaling events. Biochim Biophys Acta. 2011:1813(10):1822-6.
112. Grigorieva DV, et al. Effects of recombinant human lactoferrin on calcium signaling and functional responses of human neutrophils. Arch Biochem Biophys. 2019:675:108122.

113. Metzler KD, et al. A myeloperoxidase-containing complex regulates neutrophil elastase release and actin dynamics during NETosis. Cell Rep. 2014;8(3):883-96.

114. Maxwell PH, et al. The tumour suppressor protein VHL targets hypoxia-inducible factors for oxygen-dependent proteolysis. Nature. 1999:399(6733):271-5.

115. Sadiku P, et al. Prolyl hydroxylase 2 inactivation enhances glycogen storage and promotes excessive neutrophilic responses. J Clin Investig. 2017;127(9):3407-20

116. Mecklenburgh $\mathrm{Kl}$, et al. Involvement of a ferroprotein sensor in hypoxia-mediated inhibition of neutrophil apoptosis. Blood. 2002;100(8):3008-16.

117. Konrad FM, et al. Heme oxygenase-1 attenuates acute pulmonary inflammation by decreasing the release of segmented neutrophils from the bone marrow. Am J Physiol Lung Cell Mol Physiol. 2014;307(9):L707-17.

118. Cichon I, et al. Reduced neutrophil extracellular trap (NET) formation during systemic inflammation in mice with Menkes disease and Wilson disease: copper requirement for NET release. Front Immunol. 2019;10:3021.

119. Iwata T, et al. Ceruloplasmin induces polymorphonuclear leukocyte priming in localized aggressive periodontitis. J Periodontol. 2009:80(8):1300-6.

120. Hasan $\mathrm{R}$, Rink $\mathrm{L}$, Haase $\mathrm{H}$. Chelation of free $\mathrm{Zn}^{2+}$ impairs chemotaxis, phagocytosis, oxidative burst, degranulation, and cytokine production by neutrophil granulocytes. Biol Trace Elem Res. 2016;171(1):79-88.

121. Hasan R, Rink L, Haase H. Zinc signals in neutrophil granulocytes are required for the formation of neutrophil extracellular traps. Innate Immun. 2013;19(3):253-64.

122. Kehl-Fie TE, et al. Nutrient metal sequestration by calprotectin inhibits bacterial superoxide defense, enhancing neutrophil killing of Staphylococcus aureus. Cell Host Microbe. 2011;10(2):158-64.

123. Isaksen B, Fagerhol MK. Calprotectin inhibits matrix metalloproteinases by sequestration of zinc. Mol Pathol: MP. 2001;54(5):289-92.

124. Moroz OV et al. Both Ca 2+ and Zn 2+ are essential for S100A12 protein oligomerization and function. BMC Biochem. 2009;10(1):1-18.

125. Clark HL, et al. Zinc and manganese chelation by neutrophil S100A8/A9 (Calprotectin) limits extracellular aspergillus fumigatus hyphal growth and corneal infection. J Immunol. 2016;196(1):336-44.

126. Köse SA, Nazıroğlu M. Selenium reduces oxidative stress and calcium entry through TRPV1 channels in the neutrophils of patients with polycystic ovary syndrome. Biol Trace Elem Res. 2014;158(2):136-42.

127. Kashiwakura J, et al. Pivotal advance: IgE accelerates in vitro development of mast cells and modifies their phenotype. J Leukoc Biol. 2008;84(2):357-67.

128. Kirshenbaum AS, et al. Demonstration of the origin of human mast cells from CD34+ bone marrow progenitor cells. J Immunol. 1991;146(5):1410-5.

129. Tsai $M$, et al. The rat c-kit ligand, stem cell factor, induces the development of connective tissue-type and mucosal mast cells in vivo. Analysis by anatomical distribution, histochemistry, and protease phenotype. J Exp Med. 1991;174(1):125-31.

130. Suto $H$, et al. Mast cell-associated tnf promotes dendritic cell migration. J Immunol. 2006;176(7):4102-12.

131. Levi-Schaffer F, et al. Mouse bone marrow-derived mast cells cocultured with fibroblasts. Morphology and stimulation-induced release of histamine, leukotriene B4, leukotriene C4, and prostaglandin D2. J Immunol. 1987;139(10):3431-41

132. McCurdy JD, et al. Cutting edge: distinct toll-like receptor 2 activators selectively induce different classes of mediator production from human mast cells. J Immunol. 2003;170(4):1625-9.

133. Weller $\mathrm{CL}$, et al. Chemotactic action of prostaglandin $\mathrm{E}<\mathrm{sub}>2</$ sub $>$ on mouse mast cells acting via the $\mathrm{PGE}<\mathrm{sub}>2</$ sub $>$ receptor 3 . Proc Natl Acad Sci. 2007;104(28):11712-7.

134. Abonia JP, et al. Constitutive homing of mast cell progenitors to the intestine depends on autologous expression of the chemokine receptor CXCR2. Blood. 2005;105(11):4308-13. 
135. Dvorak AM. Ultrastructural analysis of human mast cells and basophils. Chem Immunol. 1995;61:1-33.

136. Kirshenbaum AS, et al. IL-3-dependent growth of basophil-like cells and mastlike cells from human bone marrow. J Immunol. 1989;142(7):2424-9.

137. Varricchi $G$, et al. Human mast cells and basophils-How are they similar how are they different? Immunol Rev. 2018;282(1):8-34.

138. Schwartz C, Eberle JU, Voehringer D. Basophils in inflammation. Eur J Pharmacol. 2016:778:90-5.

139. Brunner T, Heusser CH, Dahinden CA. Human peripheral blood basophils primed by interleukin 3 (IL-3) produce IL-4 in response to immunoglobulin E receptor stimulation. J Exp Med. 1993;177(3):605-11.

140. Arinobu Y, et al. Developmental checkpoints of the basophil/mast cell lineages in adult murine hematopoiesis. Proc Natl Acad Sci. 2005;102(50):18105-10.

141. Cohen $\mathrm{M}$, et al. Lung single-cell signaling interaction map reveals basophil role in macrophage imprinting. Cell. 2018;175(4):1031-1044.e18.

142. Lamkhioued B, et al. Increased expression of eotaxin in bronchoalveolar lavage and airways of asthmatics contributes to the chemotaxis of eosinophils to the site of inflammation. J Immunol. 1997;159(9):4593-601.

143. Stevens WW, et al. Detection and quantitation of eosinophils in the murine respiratory tract by flow cytometry. J Immunol Methods. 2007;327(1-2):63-74.

144. Mesnil C, et al. Lung-resident eosinophils represent a distinct regulatory eosinophil subset. J Clin Investig. 2016;126(9):3279-95.

145. Yi S, et al. Eosinophil recruitment is dynamically regulated by interplay among lung dendritic cell subsets after allergen challenge. Nat Commun. 2018;9(1):3879.

146. Drake MG, et al. Human and mouse eosinophils have antiviral activity against parainfluenza virus. Am J Respir Cell Mol Biol. 2016;55(3):387-94.

147. Palma-Carlos AG, Palma-Carlos ML, Costa AC. "Minor" hemoglobinopathies: a risk factor for asthma. Eur Ann Allergy Clin Immunol. 2005;37(5):177-82.

148. Wu Z, Holwill SD, Oliveira DB. Desferrioxamine modulates chemically induced Thelper 2-mediated autoimmunity in the rat. Clin Exp Immunol. 2004;135(2):194-9.

149. Maazi $\mathrm{H}$, et al. Iron administration reduces airway hyperreactivity and eosinophilia in a mouse model of allergic asthma. Clin Exp Immunol. 2011;166(1):80-6.

150. Arnhold J, Furtmüller PG, Obinger C, Redox properties of myeloperoxidase. 2003, Redox Rep. p. 179-186

151. Ten RM, et al. Molecular cloning of the human eosinophil peroxidase. Evidence for the existence of a peroxidase multigene family. J Exp Med. 1989;169(5):1757-69.

152. Voehringer D, van Rooijen N, Locksley RM. Eosinophils develop in distinct stages and are recruited to peripheral sites by alternatively activated macrophages. J Leukoc Biol. 2007;81(6):1434-44.

153. Sumbayev W, et al. Involvement of hypoxia-inducible factor-1 (HiF-1a) in IgE-mediated primary human basophil responses. Eur J Immunol. 2009;39(12):3511-9.

154. Ünal $M$, et al. Serum levels of antioxidant vitamins, copper, zinc and magnesium in children with chronic rhinosinusitis. J Trace Elem Med Biol. 2004;18(2):189-92.

155. Guo C-H, et al. Role of certain trace minerals in oxidative stress, inflammation, CD4/CD8 lymphocyte ratios and lung function in asthmatic patients. Ann Clin Biochem. 2011;48(4):344-51.

156. Ariaee $\mathrm{N}$, et al. Trace elements status in sera of patients with allergic asthma. Rep Biochem Mol Biol. 2016;5(1):20-5.

157. Ho LH, et al. Labile zinc and zinc transporter ZnT4 in mast cell granules: role in regulation of caspase activation and NF-kappaB translocation. J Immunol. 2004;172(12):7750-60.

158. Ugajin T, et al. Zinc-binding metallothioneins are key modulators of IL-4 production by basophils. Mol Immunol. 2015;66(2):180-8.

159. Richter $M$, et al. Zinc status modulates bronchopulmonary eosinophil infiltration in a murine model of allergic inflammation. Chest. 2003;123(3 Suppl):446s.

160. Lu H, et al. Zinc suppressed the airway inflammation in asthmatic rats: effects of zinc on generation of eotaxin, MCP-1, IL-8, IL-4, and IFN- $\gamma$. Biol Trace Elem Res. 2012;150(1-3):314-21.
161. Öhrvik H, et al. Ctr2 regulates mast cell maturation by affecting the storage and expression of tryptase and proteoglycans. J Immunol. 2015;195(8):3654-64

162. Simoni Y, et al. Human innate lymphoid cell subsets possess tissuetype based heterogeneity in phenotype and frequency. Immunity. 2018;48(5):1060.

163. Yudanin NA, et al. Spatial and temporal mapping of human innate lymphoid cells reveals elements of tissue specificity. Immunity. 2019:50(2):505-19.

164. Kärre K, et al. Selective rejection of H-2-deficient lymphoma variants suggests alternative immune defence strategy. Nature. 1986;319(6055):675-8.

165. Hart OM, et al. TLR7/8-mediated activation of human NK cells results in accessory cell-dependent IFN- $\gamma$ production. J Immunol. 2005; 175(3):1636-42.

166. Kumar $\mathrm{P}$, et al. IL-22 from conventional NK cells is epithelial regenerative and inflammation protective during influenza infection. Mucosal Immunol. 2013;6(1):69-82

167. Marquardt N, et al. Human lung natural killer cells are predominantly comprised of highly differentiated hypofunctional CD69- CD56dim cells. J Allergy Clin Immunol. 2017;139(4):1321-30.

168. Fehniger TA, et al. Differential cytokine and chemokine gene expression by human NK cells following activation with IL-18 or IL-15 in combination with IL-12: implications for the innate immune response. J Immunol. 1999;162(8):4511-20.

169. Yamamoto $Y$, et al. Lung-resident natural killer cells control pulmonary tumor growth in mice. Cancer Sci. 2018;109(9):2670-6.

170. Marquardt $\mathrm{N}$, et al. Unique transcriptional and protein-expression signature in human lung tissue-resident NK cells. Nat Commun. 2019;10(1):3841.

171. Michel T, et al. Mouse lung and spleen natural killer cells have phenotypic and functional differences, in part influenced by macrophages. PLOS ONE. 2012;7(12):e51230.

172. Cong J, Wei H. Natural killer cells in the lungs. Front Immunol. 2019:10:1416.

173. Cooper GE, et al. Human CD49a(+) lung natural killer cell cytotoxicity in response to influenza a virus. Front Immunol. 2018;9:1671.

174. Orange JS. Human natural killer cell deficiencies and susceptibility to infection. Microbes Infect. 2002;4(15):1545-58.

175. Augustin A, Kubo RT, Sim GK. Resident pulmonary lymphocytes expressing the gamma/delta T-cell receptor. Nature. 1989:340(6230):239-41.

176. Cheng M, Hu S. Lung-resident gammadelta T cells and their roles in lung diseases. Immunology. 2017;151(4):375-84.

177. Barlow JL, McKenzie ANJ. Innate lymphoid cells of the lung. Annu Rev Physiol. 2019;81:429-52.

178. Trottein F, Paget C. Natural killer T cells and mucosal-associated invariant t cells in lung infections. Front Immunol. 2018;9:1750

179. Metelitsa LS, et al. Human NKT cells mediate antitumor cytotoxicity directly by recognizing target cell CD1d with bound ligand or indirectly by producing IL-2 to activate NK cells. J Immunol. 2001;167(6):3114-22.

180. Lee YJ, et al. Tissue-specific distribution of iNKT cells impacts their cytokine response. Immunity. 2015:43(3):566-78.

181. Thanabalasuriar $A$, et al. iNKT cell emigration out of the lung vasculature requires neutrophils and monocyte-derived dendritic cells in inflammation. Cell Rep. 2016;16(12):3260-72.

182. Gold MC, et al. Human mucosal associated invariant T cells detect bacterially infected cells. PLoS Biol. 2010;8(6):e1000407.

183. Marçais A, et al. The metabolic checkpoint kinase mTOR is essential for IL-15 signaling during the development and activation of NK cells. Nat Immunol. 2014;15(8):749-57.

184. Keating SE, et al. Metabolic reprogramming supports IFN- $\gamma$ production by CD56<sup>bright</sup> NK Cells. J Immunol. 2016;196(6):2552-60.

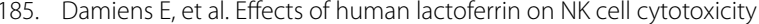
against haematopoietic and epithelial tumour cells. Biochim Biophys Acta. 1998:1402(3):277-87.

186. Hua Y, et al. Iron overload may promote alteration of NK cells and hematopoietic stem/progenitor cells by JNK and P38 pathway in myelodysplastic syndromes. Int J Hematol. 2017;106(2):248-57.

187. Sottile R, et al. Iron and ferritin modulate MHC class I expression and NK cell recognition. Front Immunol. 2019;10:224. 
188. Kumar S, et al. Zinc-induced polymerization of killer-cell lg-like receptor into filaments promotes its inhibitory function at cytotoxic immunological synapses. Mol Cell. 2016;62(1):21-33.

189. Muzzioli M, et al. Zinc improves the development of human CD34+ cell progenitors towards NK cells and increases the expression of GATA-3 transcription factor in young and old ages. Biogerontology. 2009;10(5):593-604

190. Wessels I, Maywald M, Rink L. Zinc as a gatekeeper of immune function. Nutrients, 2017; 9(12)

191. Mace EM, et al. A dual role for talin in NK cell cytotoxicity: activation of LFA-1-mediated cell adhesion and polarization of NK cells. J Immunol. 2009;182(2):948-56.

192. LV M, et al. Manganese is critical for antitumor immune responses via CGAS-STING and improves the efficacy of clinical immunotherapy. Cell Res. 2020;30(11):966-79.

193. Kiremidjian-Schumacher $L$, et al. Supplementation with selenium augments the functions of natural killer and lymphokine-activated killer cells. Biol Trace Elem Res. 1996;52(3):227-39.

194. Brekelmans $P$, et al. Inhibition of proliferation and differentiation during early $T$ cell development by anti-transferrin receptor antibody. Eur J Immunol. 1994;24(11):2896-902.

195. Mincheva-Nilsson L, Hammarstrom S, Hammarstrom ML. Activated human gamma delta T lymphocytes express functional lactoferrin receptors. Scand J Immunol. 1997;46(6):609-18.

196. Vargas Buonfiglio LG, et al. Airway surface liquid from smokers promotes bacterial growth and biofilm formation via iron-lactoferrin imbalance. Respir Res. 2018;19(1):42.

197. Reuben A, et al. The hemochromatosis protein HFE 20 years later: an emerging role in antigen presentation and in the immune system. Immun Inflamm Dis. 2017;5(3):218-32.

198. French JD, et al. Gammadelta T lymphocyte homeostasis is negatively regulated by beta2-microglobulin. J Immunol. 2009;182(4):1892-900.

199. de Sousa M, et al. Iron overload in $\beta 2$-microglobulin-deficient mice. Immunol Lett. 1994;39(2):105-11.

200. Maia ML, et al. Invariant natural killer T cells are reduced in hereditary hemochromatosis patients. J Clin Immunol. 2015;35(1):68-74.

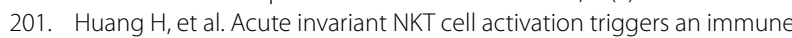
response that drives prominent changes in iron homeostasis. Sci Rep. 2020;10(1):21026.

202. Pabst R, Tschernig T. Lymphocytes in the lung: an often neglected cell. Numbers, characterization and compartmentalization. Anat Embryol (Berl). 1995;192(4):293-9.

203. Hwang JY, Randall TD, Silva-Sanchez A. Inducible bronchus-associated lymphoid tissue: taming inflammation in the lung. Front Immunol. 2016;7:258.

204. Hogg JC, et al. The nature of small-airway obstruction in chronic obstructive pulmonary disease. N Engl J Med. 2004;350(26):2645-53.

205. Belz GT, et al. Tissue-resident lymphocytes: weaponized sentinels at barrier surfaces. F1000Res, 2020; 9.

206. Niedzielska M, et al. Differential gene expression in human tissue resident regulatory $T$ cells from lung, colon, and blood. Oncotarget. 2018;9(90):36166-84.

207. Lu C, et al. Memory regulatory T cells home to the lung and control influenza A virus infection. Immunol Cell Biol. 2019;97(9):774-86.

208. Ichikawa T, et al. CD103(hi) Treg cells constrain lung fibrosis induced by CD103(lo) tissue-resident pathogenic CD4 T cells. Nat Immunol. 2019;20(11):1469-80.

209. Weiss $\mathrm{G}$. Modification of iron regulation by the inflammatory response. Best Pract Res Clin Haematol. 2005;18(2):183-201.

210. Brock JH. The effect of iron and transferrin on the response of serumfree cultures of mouse lymphocytes to concanavalin A and lipopolysaccharide. Immunology. 1981;43(2):387-92.

211. Mateos F, Brock JH, Perez-Arellano JL. Iron metabolism in the lower respiratory tract. Thorax. 1998;53(7):594-600.

212. Stoffel NU, et al. Iron deficiency anemia at time of vaccination predicts decreased vaccine response and iron supplementation at time of vaccination increases humoral vaccine response: a birth cohort study and a randomized trial follow-up study in Kenyan infants. Front Immunol. 2020;11:1313

213. Frost JN, et al. Hepcidin-mediated hypoferremia disrupts immune responses to vaccination and infection. Med. 2021;2(2):164-179.e12.
214. Jabara HH, et al. A missense mutation in TFRC, encoding transferrin receptor 1 , causes combined immunodeficiency. Nat Genet. 2016;48(1):74-8.

215. Cunningham-Rundles $S$, et al. Effect of transfusional iron overload on immune response. J Infect Dis. 2000;182(Suppl 1):S115-21.

216. Ehrlich R, Lemonnier FA. HFE-a novel nonclassical class I molecule that is involved in iron metabolism. Immunity. 2000;13(5):585-8.

217. Matsushita M, et al. T cell lipid peroxidation induces ferroptosis and prevents immunity to infection. J Exp Med. 2015;212(4):555-68.

218. Roy M, et al. Supplementation with selenium and human immune cell functions. I. Effect on lymphocyte proliferation and interleukin 2 receptor expression. Biol Trace Elem Res. 1994;41(1-2):103-14.

219. Elmadfa I, Meyer AL. The role of the status of selected micronutrients in shaping the immune function. Endocr Metab Immune Disord Drug Targets. 2019;19(8):1100-15.

220. Budden KF, et al. Functional effects of the microbiota in chronic respiratory disease. Lancet Respir Med. 2019;7(10):907-20.

221. Dickson RP, et al. Bacterial topography of the healthy human lower respiratory tract. mBio, 2017; 8(1).

222. Dickson RP, et al. Changes in the lung microbiome following lung transplantation include the emergence of two distinct Pseudomonas species with distinct clinical associations. PLOS ONE. 2014;9(5):e97214.

223. Segal LN, et al. Enrichment of lung microbiome with supraglottic taxa is associated with increased pulmonary inflammation. Microbiome. 2013;1(1):19.

224. Morris A, et al. Comparison of the respiratory microbiome in healthy nonsmokers and smokers. Am J Respir Crit Care Med. 2013;187(10):1067-75.

225. Andrews SC, Robinson AK, Rodríguez-Quiñones F. Bacterial iron homeostasis. FEMS Microbiol Rev. 2003:27(2-3):215-37.

226. Andrei A, et al. Cu Homeostasis in Bacteria: The Ins and Outs. Membranes (Basel), 2020; 10(9).

227. Mevissen-Verhage EA, et al. Effect of iron on neonatal gut flora during the first three months of life. Eur J Clin Microbiol. 1985;4(3):273-8.

228. Dostal A, et al. Low iron availability in continuous in vitro colonic fermentations induces strong dysbiosis of the child gut microbial consortium and a decrease in main metabolites. FEMS Microbiol Ecol. 2013;83(1):161-75.

229. Tompkins GR, et al. The effects of dietary ferric iron and iron deprivation on the bacterial composition of the mouse intestine. Curr Microbiol. 2001;43(1):38-42.

230. Dostal A, et al. Iron depletion and repletion with ferrous sulfate or electrolytic iron modifies the composition and metabolic activity of the gut microbiota in rats. J Nutr. 2012;142(2):271-7.

231. Buhnik-Rosenblau K, et al. Genetic modification of iron metabolism in mice affects the gut microbiota. Biometals. 2012;25(5):883-92.

232. Song $M$, et al. Dietary copper-fructose interactions alter gut microbial activity in male rats. Am J Physiol Gastrointest Liver Physiol. 2018;314(1):G119-g130.

233. Zhang Y, et al. Effect of dietary copper on intestinal microbiota and antimicrobial resistance profiles of Escherichia coli in weaned piglets. Front Microbiol. 2019;10:2808.

234. Pieper R, et al. Concentration and chemical form of dietary zinc shape the porcine colon microbiome, its functional capacity and antibiotic resistance gene repertoire. Isme j. 2020;14(11):2783-93.

235. Foligné $B$, et al. High-dose dietary supplementation with zinc prevents gut inflammation: investigation of the role of metallothioneins and beyond by transcriptomic and metagenomic studies. Faseb j. 2020;34(9):12615-33.

236. Podany A, et al. Excess dietary zinc intake in neonatal mice causes oxidative stress and alters intestinal host-microbe interactions. Mol Nutr Food Res. 2019;63(3):e1800947.

237. Gifford $\mathrm{AH}$, et al. Iron supplementation does not worsen respiratory health or alter the sputum microbiome in cystic fibrosis. J Cyst Fibros. 2014;13(3):311-8.

238. Rooks MG, Garrett WS. Gut microbiota, metabolites and host immunity. Nat Rev Immunol. 2016;16(6):341-52.

239. Sharma NS, et al. Differences in airway microbiome and metabolome of single lung transplant recipients. Respir Res. 2020;21(1):104. 
240. O'Dwyer DN, et al. Lung dysbiosis, inflammation, and injury in hematopoietic cell transplantation. Am J Respir Crit Care Med. 2018;198(10):1312-21.

241. Cevhertas $L$, et al. Advances and recent developments in asthma in 2020. Allergy. 2020

242. Lambrecht BN, Hammad H. The immunology of asthma. Nat Immunol. 2015;16(1):45-56

243. Woodruff PG, et al. T-helper type 2-driven inflammation defines major subphenotypes of asthma. Am J Respir Crit Care Med. 2009;180(5):388-95.

244. Barlow JL, et al. Innate IL-13-producing nuocytes arise during allergic lung inflammation and contribute to airways hyperreactivity. J Allergy Clin Immunol. 2012;129(1):191-8.

245. Zhao J, Lloyd CM, Noble A. Th17 responses in chronic allergic airway inflammation abrogate regulatory T-cell-mediated tolerance and contribute to airway remodeling. Mucosal Immunol. 2013;6(2):335-46.

246. Raymond M, et al. Selective control of SIRP-alpha-positive airway dendritic cell trafficking through CD47 is critical for the development of $\mathrm{T}(\mathrm{H}) 2$-mediated allergic inflammation. J Allergy Clin Immunol. 2009:124(6):1333-42.

247. Williams JW, et al. Transcription factor IRF4 drives dendritic cells to promote Th2 differentiation. Nat Commun. 2013;4:2990.

248. Barcik W, et al. The role of lung and gut microbiota in the pathology of asthma. Immunity. 2020;52(2):241-55.

249. Thorsen J, et al. Infant airway microbiota and topical immune perturbations in the origins of childhood asthma. Nat Commun. 2019;10(1):5001.

250. Huang YJ, et al. The airway microbiome in patients with severe asthma: associations with disease features and severity. J Allergy Clin Immunol. 2015;136(4):874-84

251. Durack J, et al. Features of the bronchial bacterial microbiome associated with atopy, asthma, and responsiveness to inhaled corticosteroid treatment. J Allergy Clin Immunol. 2017;140(1):63-75.

252. Arrieta MC, et al. Associations between infant fungal and bacterial dysbiosis and childhood atopic wheeze in a nonindustrialized setting. J Allergy Clin Immunol. 2018;142(2):424-34.

253. Trompette A, et al. Gut microbiota metabolism of dietary fiber influences allergic airway disease and hematopoiesis. Nat Med. 2014;20(2):159-66.

254. Roduit C, et al. High levels of butyrate and propionate in early life are associated with protection against atopy. Allergy. 2019;74(4):799-809.

255. McLoughlin R, et al. Soluble fibre supplementation with and without a probiotic in adults with asthma: a 7-day randomised, double blind, three way cross-over trial. EBioMedicine. 2019;46:473-85.

256. Brigham EP, et al. Iron status is associated with asthma and lung function in US women. PLoS ONE. 2015;10(2):e0117545.

257. Drury KE, Schaeffer M, Silverberg J. Association between atopic disease and anemia in US children. JAMA Pediatr. 2016;170(1):29-34.

258. Thorson JA, et al. Role of iron in T cell activation: TH1 clones differ from $\mathrm{TH} 2$ clones in their sensitivity to inhibition of DNA synthesis caused by IgG Mabs against the transferrin receptor and the iron chelator deferoxamine. Cell Immunol. 1991;134(1):126-37.

259. Leung S, et al. Differential inhibition of inducible T cell cytokine secretion by potent iron chelators. J Biomol Screen. 2005;10(2):157-67.

260. Ali MK, et al. Crucial role for lung iron level and regulation in the pathogenesis and severity of asthma. Eur Respir J. 2020; 55(4).

261. Roth-Walter $F$, et al. Bet $\vee 1$ from birch pollen is a lipocalin-like protein acting as allergen only when devoid of iron by promoting Th2 lymphocytes. J Biol Chem. 2014;289(25):17416-21.

262. Roth-Walter $\mathrm{F}$, et al. The major cow milk allergen Bos d 5 manipulates T-helper cells depending on its load with siderophore-bound iron. PLoS ONE. 2014;9(8):e104803.

263. Dostal A, et al. Effects of iron supplementation on dominant bacterial groups in the gut, faecal SCFA and gut inflammation: a randomised, placebo-controlled intervention trial in South African children. Br J Nutr. 2014:112(4):547-56.

264. Hoffmann PR, et al. A role for dietary selenium and selenoproteins in allergic airway inflammation. J Immunol. 2007;179(5):3258-67.

265. Vural H, et al. Concentrations of copper, zinc and various elements in serum of patients with bronchial asthma. J Trace Elem Med Biol. 2000:14(2):88-91.
266. Yates DH. Role of exhaled nitric oxide in asthma. Immunol Cell Biol. 2001;79(2):178-90.

267. Gaston B, et al. Bronchodilator S-nitrosothiol deficiency in asthmatic respiratory failure. Lancet. 1998;351(9112):1317-9.

268. GBD 2015 Chronic Respiratory Disease Collaborators. Global, regional, and national deaths, prevalence, disability-adjusted life years, and years lived with disability for chronic obstructive pulmonary disease and asthma, 1990-2015: a systematic analysis for the Global Burden of Disease Study 2015. Lancet Respir Med. 2017:5(9):691-706.

269. Vogelmeier CF, et al. Global strategy for the diagnosis, management, and prevention of chronic obstructive lung disease 2017 report. GOLD executive summary. Am J Respir Crit Care Med. 2017;195(5):557-82.

270. Postma DS, Bush A, van den Berge M. Risk factors and early origins of chronic obstructive pulmonary disease. Lancet. 2015;385(9971):899-909.

271. GrashoffWF, et al. Chronic obstructive pulmonary disease: role of bronchiolar mast cells and macrophages. Am J Pathol. 1997;151(6):1785-90.

272. Finkelstein $\mathrm{R}$, et al. Alveolar inflammation and its relation to emphysema in smokers. Am J Respir Crit Care Med. 1995;152(5 Pt 1):1666-72.

273. van Zyl-Smit RN, et al. Cigarette smoke impairs cytokine responses and BCG containment in alveolar macrophages. Thorax. 2014;69(4):363-70.

274. Phipps JC, et al. Cigarette smoke exposure impairs pulmonary bacterial clearance and alveolar macrophage complement-mediated phagocytosis of Streptococcus pneumoniae. Infect Immun. 2010;78(3):1214-20.

275. Drannik AG, et al. Impact of cigarette smoke on clearance and inflammation after Pseudomonas aeruginosa infection. Am J Respir Crit Care Med. 2004;170(11):1164-71.

276. Hodge S, et al. Alveolar macrophages from subjects with chronic obstructive pulmonary disease are deficient in their ability to phagocytose apoptotic airway epithelial cells. Immunol Cell Biol. 2003:81(4):289-96.

277. Retamales I, et al. Amplification of inflammation in emphysema and its association with latent adenoviral infection. Am J Respir Crit Care Med. 2001;164(3):469-73.

278. Di Stefano A, et al. Severity of airflow limitation is associated with severity of airway inflammation in smokers. Am J Respir Crit Care Med. 1998;158(4):1277-85.

279. Jasper $A E$, et al. Understanding the role of neutrophils in chronic inflammatory airway disease. F1000Res, 2019; 8.

280. Kim VL, et al. Impact and associations of eosinophilic inflammation in COPD: analysis of the AERIS cohort. Eur Respir J. 2017; 50(4).

281. Kolsum U, et al. Blood and sputum eosinophils in COPD; relationship with bacterial load. Respir Res. 2017;18(1):88.

282. Wilkinson TMA, et al. A prospective, observational cohort study of the seasonal dynamics of airway pathogens in the aetiology of exacerbations in COPD. Thorax. 2017:72(10):919-27.

283. Wang Z, et al. Airway host-microbiome interactions in chronic obstructive pulmonary disease. Respir Res. 2019;20(1):113.

284. Opron $\mathrm{K}$, et al. Lung microbiota associations with clinical features of COPD in the SPIROMICS cohort. NPJ Biofilms Microbiomes. 2021;7(1):14

285. Wang $Z$, et al. A refined view of airway microbiome in chronic obstructive pulmonary disease at species and strain-levels. Front Microbiol. 2020;11:1758

286. Pragman AA, et al. The lung microbiome in moderate and severe chronic obstructive pulmonary disease. PLOS ONE. 2012;7(10):e47305.

287. Dicker AJ, et al. The sputum microbiome, airway inflammation, and mortality in chronic obstructive pulmonary disease. J Allergy Clin Immunol. 2021;147(1):158-67.

288. Ghebre MA, et al. Biological exacerbation clusters demonstrate asthma and chronic obstructive pulmonary disease overlap with distinct mediator and microbiome profiles. J Allergy Clin Immunol. 2018;141(6):20272036.e12.

289. Haldar K, et al. The sputum microbiome is distinct between COPD and health, independent of smoking history. Respir Res. 2020;21(1):183.

290. Ren L, et al. Transcriptionally active lung microbiome and its association with bacterial biomass and host inflammatory status. mSystems. 2018; 3(5).

291. Sze MA, et al. Host response to the lung microbiome in chronic obstructive pulmonary disease. Am J Respir Crit Care Med. 2015;192(4):438-45. 
292. Huang YJ, et al. Airway microbiome dynamics in exacerbations of chronic obstructive pulmonary disease. J Clin Microbiol. 2014;52(8):2813-23.

293. Wang Z, et al. Lung microbiome dynamics in COPD exacerbations. Eur Respir J. 2016;47(4):1082-92

294. Taylor SL, et al. Inflammatory phenotypes in patients with severe asthma are associated with distinct airway microbiology. J Allergy Clin Immunol. 2018;141(1):94-103.e15.

295. Bowerman KL, et al. Disease-associated gut microbiome and metabolome changes in patients with chronic obstructive pulmonary disease. Nat Commun. 2020;11(1):5886.

296. Wu Y, Luo Z, Liu C. Variations in fecal microbial profiles of acute exacerbations and stable chronic obstructive pulmonary disease. Life Sci. 2021;265:118738

297. Jang YO, et al. Fecal microbial transplantation and a high fiber diet attenuates emphysema development by suppressing inflammation and apoptosis. Exp Mol Med. 2020;52(7):1128-39.

298. DeMeo DL, et al. Integration of genomic and genetic approaches implicates IREB2 as a COPD susceptibility gene. Am J Hum Genet. 2009;85(4):493-502

299. Chappell SL, et al. The role of IREB2 and transforming growth factor beta-1 genetic variants in COPD: a replication case-control study. BMC Med Genet. 2011;12:24

300. Du Y, Xue Y, Xiao W. Association of IREB2 gene rs2568494 polymorphism with risk of chronic obstructive pulmonary disease: a meta-analysis. Med Sci Monit. 2016;22:177-82.

301. Hardin $M$, et al. CHRNA3/5, IREB2, and ADCY2 are associated with severe chronic obstructive pulmonary disease in Poland. Am J Respir Cell Mol Biol. 2012;47(2):203-8.

302. Ding Y, et al. Association of genetic polymorphisms with chronic obstructive pulmonary disease in the Hainan population: a case-control study. Int J Chron Obstruct Pulmon Dis. 2015;10:7-13.

303. Corhay JL, et al. Iron content in human alveolar macrophages. Eur Respir J. 1992;5(7):804-9.

304. Ghio AJ, et al. Particulate matter in cigarette smoke alters iron homeostasis to produce a biological effect. Am J Respir Crit Care Med. 2008;178(11):1130-8.

305. Thompson AB, et al. Lower respiratory tract iron burden is increased in association with cigarette smoking. J Lab Clin Med. 1991;117(6):493-9.

306. McGowan SE, Henley SA. Iron and ferritin contents and distribution in human alveolar macrophages. J Lab Clin Med. 1988;111(6):611-7.

307. Philippot $\mathrm{Q}$, et al. Increased iron sequestration in alveolar macrophages in chronic obstructive pulmonary disease. PLoS ONE. 2014;9(5):e96285.

308. Wesselius LJ, Flowers $\mathrm{CH}$, Skikne BS. Alveolar macrophage content of isoferritins and transferrin. Comparison of nonsmokers and smokers with and without chronic airflow obstruction. Am Rev Respir Dis. 1992;145(2 pt 1):311-6.

309. Wesselius LJ, Nelson ME, Skikne BS. Increased release of ferritin and iron by iron-loaded alveolar macrophages in cigarette smokers. Am J Respir Crit Care Med. 1994;150(3):690-5.

310. Nelson ME, O'Brien-Ladner AR, Wesselius LJ. Regional variation in iron and iron-binding proteins within the lungs of smokers. Am J Respir Crit Care Med. 1996:153(4 Pt 1):1353-8.

311. Mumby S, et al. Decreased breath excretion of redox active iron in COPD: a protective failure? Eur Respir J, 2015.

312. Zhang WZ, et al. Increased airway iron parameters and risk for exacerbation in COPD: an analysis from SPIROMICS. Sci Rep. 2020; 10(1).

313. John $\mathrm{M}$, et al. Prevalence of anemia in chronic obstructive pulmonary disease: comparison to other chronic diseases. Int J Cardiol. 2006;111(3):365-70

314. Martinez-Rivera C, et al. Anemia is a mortality predictor in hospitalized patients for COPD exacerbation. COPD. 2012;9(3):243-50.

315. Boutou AK, et al. Anemia and survival in chronic obstructive pulmonary disease: a dichotomous rather than a continuous predictor. Respiration. 2013;85(2):126-31.

316. Nickol AH, et al. A cross-sectional study of the prevalence and associations of iron deficiency in a cohort of patients with chronic obstructive pulmonary disease. BMJ Open. 2015;5(7):e007911.

317. Mackay AJ, Loza M, Branigan BS, Baribaud F, George SN, Donaldson GC, Wedzicha JA. Serum amyloid A and hepcidin elevations characterise bacterial-associated exacerbations in frequently exacerbating COPD patients. Am J Respir Critical Care Med. 2015;191:A6336.

318. Tandara L, et al. Systemic inflammation up-regulates serum hepcidin in exacerbations and stabile chronic obstructive pulmonary disease. Clin Biochem. 2015:48(18):1252-7.

319. Cloonan SM, et al. Mitochondrial iron chelation ameliorates cigarette smoke-induced bronchitis and emphysema in mice. Nat Med. 2016;22(2):163-74.

320. Sato $K$, et al. Effect of iron deficiency on a murine model of smokeinduced emphysema. Am J Respir Cell Mol Biol. 2020;62(5):588-97.

321. Santer $\mathrm{P}$, et al. Intravenous iron and chronic obstructive pulmonary disease: a randomised controlled trial. BMJ Open Respir Res, 2020; 7(1).

322. Yoshida M, et al. Involvement of cigarette smoke-induced epithelial cell ferroptosis in COPD pathogenesis. Nat Commun. 2019;10(1):3145.

323. Karadag F, et al. Trace elements as a component of oxidative stress in COPD. Respirology. 2004;9(1):33-7.

324. Lin YS, et al. Cigarette smoking, cadmium exposure, and zinc intake on obstructive lung disorder. Respir Res. 2010;11(1):53.

325. Napolitano JR, et al. Cadmium-mediated toxicity of lung epithelia is enhanced through NF-kB-mediated transcriptional activation of the human zinc transporter ZIP8. Am J Physiol Lung Cell Mol Physiol. 2012;302(9):L909-18.

326. Roscioli E, et al. Zinc deficiency as a codeterminant for airway epithelial barrier dysfunction in an ex vivo model of COPD. Int J Chron Obstruct Pulmon Dis. 2017:12:3503-10.

327. Roscioli E, et al. The uncoupling of autophagy and zinc homeostasis in airway epithelial cells as a fundamental contributor to COPD. Am J Physiol Lung Cell Mol Physiol. 2017;313(3):L453-1465.

328. Knoell DL, et al. Imbalance in zinc homeostasis enhances lung Tissue Loss following cigarette smoke exposure. J Trace Elem Med Biol. 2020:60:126483.

329. Knoell DL, et al. Insufficient zinc intake enhances lung inflammation in response to agricultural organic dust exposure. J Nutr Biochem. 2019;70:56-64.

330. Lang CJ, et al. Dietary zinc mediates inflammation and protects against wasting and metabolic derangement caused by sustained cigarette smoke exposure in mice. Biometals. 2011;24(1):23-39.

331. Grasseschi RM, et al. Cadmium accumulation and detoxification by alveolar macrophages of cigarette smokers. Chest. 2003;124(5):1924-8.

332. Soskel NT, et al. A copper-deficient, zinc-supplemented diet produces emphysema in pigs. Am Rev Respir Dis. 1982;126(2):316-25.

333. O'Dell BL, et al. The lung of the copper-deficient rat. A model for developmental pulmonary emphysema. Am J Pathol. 1978;91(3):413-32.

334. Mizuno S, et al. Copper deficiency induced emphysema is associated with focal adhesion kinase inactivation. PLoS ONE. 2012;7(1):e30678.

335. Mutti A, et al. Exhaled metallic elements and serum pneumoproteins in asymptomatic smokers and patients with COPD or asthma. Chest. 2006;129(5):1288-97.

336. Janssen $\mathrm{R}$, et al. Copper-heparin inhalation therapy to repair emphysema: a scientific rationale. Int J Chron Obstruct Pulmon Dis. 2019:14:2587-602

337. Grange DK, et al. Severe bilateral panlobular emphysema and pulmonary arterial hypoplasia: unusual manifestations of Menkes disease. Am J Med Genet A. 2005;139a(2):151-5.

338. Janssen $\mathrm{R}$, et al. Copper as the most likely pathogenic divergence factor between lung fibrosis and emphysema. Med Hypotheses. 2018;120:49-54.

339. Agler $\mathrm{AH}$, et al. Differential expression of vitamin $\mathrm{E}$ and seleniumresponsive genes by disease severity in chronic obstructive pulmonary disease. COPD. 2013;10(4):450-8.

340. McKeever TM, et al. A multivariate analysis of serum nutrient levels and lung function. Respir Res. 2008;9(1):67.

341. Ates Alkan F, et al. Analysis of the relationship between hemorheologic parameters, aluminum, manganese, and selenium in smokers. Biol Trace Elem Res. 2019;187(1):22-31.

342. Hassan F, et al. Accumulation of metals in GOLD4 COPD lungs is associated with decreased CFTR levels. Respir Res. 2014;15(1):69.

343. Ratjen F, et al. Cystic fibrosis. Nature Rev Dis Primers. 2015;1(1):15010.

344. Einarsson GG, et al. Community analysis and co-occurrence patterns in airway microbial communities during health and disease. ERJ Open Res. 2019; 5(3). 
345. Madan JC, et al. Serial analysis of the gut and respiratory microbiome in cystic fibrosis in infancy: interaction between intestinal and respiratory tracts and impact of nutritional exposures. mBio, 2012; 3(4).

346. Frayman KB, et al. The lower airway microbiota in early cystic fibrosis lung disease: a longitudinal analysis. Thorax. 2017;72(12):1104-12.

347. Frayman KB, et al. Differences in the lower airway microbiota of infants with and without cystic fibrosis. J Cyst Fibros. 2019;18(5):646-52

348. Hoen AG, et al. Associations between gut microbial colonization in early life and respiratory outcomes in cystic fibrosis. J Pediatr. 2015;167(1):138-47 (e1-3)

349. Jorth $\mathrm{P}$, et al. Direct lung sampling indicates that established pathogens dominate early infections in children with cystic fibrosis. Cell Rep. 2019;27(4):1190-1204.e3.

350. Goddard AF, et al. Direct sampling of cystic fibrosis lungs indicates that DNA-based analyses of upper-airway specimens can misrepresent lung microbiota. Proc Natl Acad Sci U S A. 2012;109(34):13769-74.

351. Fodor AA, et al. The adult cystic fibrosis airway microbiota is stable over time and infection type, and highly resilient to antibiotic treatment of exacerbations. PLoS ONE. 2012;7(9):e45001.

352. Zemanick ET, et al. Airway microbiota across age and disease spectrum in cystic fibrosis. Eur Respir J, 2017; 50(5).

353. Coburn B, et al. Lung microbiota across age and disease stage in cystic fibrosis. Sci Rep. 2015;5:10241.

354. Carmody LA, et al. Changes in cystic fibrosis airway microbiota at pulmonary exacerbation. Ann Am Thorac Soc. 2013;10(3):179-87.

355. Sherrard LJ, et al. Assessment of stability and fluctuations of cultured lower airway bacterial communities in people with cystic fibrosis. J Cyst Fibros. 2019;18(6):808-16.

356. Zemanick ET, et al. Inflammation and airway microbiota during cystic fibrosis pulmonary exacerbations. PLoS ONE. 2013;8(4):e62917.

357. Valentini TD, et al. Bioorthogonal non-canonical amino acid tagging reveals translationally active subpopulations of the cystic fibrosis lung microbiota. Nat Commun. 2020;11(1):2287.

358. Pond MN, Morton AM, Conway SP. Functional iron deficiency in adults with cystic fibrosis. Respir Med. 1996;90(7):409-13.

359. Kelleher J, et al. Essential element nutritional status in cystic fibrosis. Hum Nutr Appl Nutr. 1986;40(2):79-84.

360. Ehrhardt P, Miller MG, Littlewood JM. Iron deficiency in cystic fibrosis. Arch Dis Child. 1987;62(2):185-7.

361. Keevil B, et al. Assessment of iron status in cystic fibrosis patients. Ann Clin Biochem. 2000;37(Pt 5):662-5.

362. Fischer $R$, et al. Lung disease severity, chronic inflammation, iron deficiency, and erythropoietin response in adults with cystic fibrosis. Pediatr Pulmonol. 2007;42(12):1193-7.

363. von Drygalski A, Biller J. Anemia in cystic fibrosis: incidence, mechanisms, and association with pulmonary function and vitamin deficiency. Nutr Clin Pract. 2008;23(5):557-63.

364. Reid DW, et al. Iron deficiency in cystic fibrosis: relationship to lung disease severity and chronic Pseudomonas aeruginosa infection. Chest. 2002;121(1):48-54.

365. Stites SW, et al. Increased iron and ferritin content of sputum from patients with cystic fibrosis or chronic bronchitis. Chest. 1998;114(3):814-9.

366. Reid DW, et al. Increased airway iron as a potential factor in the persistence of Pseudomonas aeruginosa infection in cystic fibrosis. Eur Respir J. 2007;30(2):286-92.

367. Reid DW, et al. Airway iron and iron-regulatory cytokines in cystic fibrosis. Eur Respir J. 2004;24(2):286-91.

368. Hunter RC, et al. Ferrous iron is a significant component of bioavailable iron in cystic fibrosis airways. mBio, 2013; 4(4).

369. Hazlett HF, et al. Altered iron metabolism in cystic fibrosis macrophages: the impact of CFTR modulators and implications for Pseudomonas aeruginosa survival. Sci Rep. 2020;10(1):10935.

370. Moreau-Marquis S, et al. The $\triangle F 508-C F T R$ mutation results in increased biofilm formation by Pseudomonas aeruginosa by increasing iron availability. Am J Physiol-Lung Cell Mol Physiol. 2008;295(1):L25-37.

371. Chillappagari S, et al. Impaired TLR4 and HIF expression in cystic fibrosis bronchial epithelial cells downregulates hemeoxygenase-1 and alters iron homeostasis in vitro. Am J Physiol Lung Cell Mol Physiol. 2014;307(10):L791-9.
372. Hendricks MR, et al. Respiratory syncytial virus infection enhances Pseudomonas aeruginosa biofilm growth through dysregulation of nutritional immunity. Proc Natl Acad Sci U S A. 2016;113(6):1642-7.

373. Goss $\mathrm{CH}$, et al. Gallium disrupts bacterial iron metabolism and has therapeutic effects in mice and humans with lung infections. Sci Transl Med. 2018; 10(460).

374. Abdalla MY, et al. Gallium compounds exhibit potential as new therapeutic agents against Mycobacterium abscessus. Antimicrob Agents Chemother. 2015:59(8):4826-34.

375. Damphousse V, et al. Plasma zinc in adults with cystic fibrosis: correlations with clinical outcomes. J Trace Elem Med Biol. 2014;28(1):60-4.

376. Krebs NF, et al. Low plasma zinc concentrations in young infants with cystic fibrosis. J Pediatr. 1998;133(6):761-4.

377. Abdulhamid I, et al. Effect of zinc supplementation on respiratory tract infections in children with cystic fibrosis. Pediatr Pulmonol. 2008;43(3):281-7.

378. Safai-Kutti S, et al. Zinc therapy in children with cystic fibrosis. Beitr Infusionsther. 1991;27:104-14

379. Van Biervliet S, et al. The effect of zinc supplements in cystic fibrosis patients. Ann Nutr Metab. 2008;52(2):152-6.

380. Ataee P, et al. Effect of supplementary zinc on body mass index, pulmonary function and hospitalization in children with cystic fibrosis. Turk J Pediatr. 2014;56(2):127-32.

381. Sharma G, et al. Zinc supplementation for one year among children with cystic fibrosis does not decrease pulmonary infection. Respir Care. 2016;61(1):78-84

382. Gray RD, et al. Sputum trace metals are biomarkers of inflammatory and suppurative lung disease. Chest. 2010;137(3):635-41.

383. Smith DJ, et al. Elevated metal concentrations in the CF airway correlate with cellular injury and disease severity. J Cyst Fibros. 2014;13(3):289-95.

384. Wakeman CA, et al. The innate immune protein calprotectin promotes Pseudomonas aeruginosa and Staphylococcus aureus interaction. Nat Commun. 2016;7(1):11951.

385. Percival SS, Bowser E, Wagner M. Reduced copper enzyme activities in blood cells of children with cystic fibrosis. Am J Clin Nutr. 1995;62(3):633-8.

386. Percival SS, et al. Altered copper status in adult men with cystic fibrosis. J Am Coll Nutr. 1999;18(6):614-9.

387. Best $\mathrm{K}$, et al. Copper enzyme activities in cystic fibrosis before and after copper supplementation plus or minus zinc. Metabolism. 2004;53(1):37-41.

388. Dworkin B, et al. Low blood selenium levels in patients with cystic fibrosis compared to controls and healthy adults. JPEN J Parenter Enteral Nutr. 1987;11(1):38-41.

389. Stead RJ, et al. Selenium deficiency and possible increased risk of carcinoma in adults with cystic fibrosis. Lancet. 1985;2(8460):862-3.

390. Nève J, et al. Erythrocyte and plasma trace element levels in clinical assessments : Zinc, copper, and selenium in normals and patients with Down's syndrome and cystic fibrosis. Biol Trace Elem Res. 1983;5(2):75-9.

391. Michalke B. Selenium speciation in human serum of cystic fibrosis patients compared to serum from healthy persons. J Chromatogr A. 2004:1058(1-2):203-8.

392. Chalmers JD, et al. Bronchiectasis. Nat Rev Dis Primers. 2018;4(1):45.

393. Woo TE, et al. A longitudinal characterization of the non-cystic fibrosis bronchiectasis airway microbiome. Sci Rep. 2019;9(1):6871.

394. Rogers GB, et al. Clinical measures of disease in adult non-CF bronchiectasis correlate with airway microbiota composition. Thorax. 2013;68(8):731-7.

395. Cox MJ, et al. Longitudinal assessment of sputum microbiome by sequencing of the 16S rRNA gene in non-cystic fibrosis bronchiectasis patients. PLoS ONE. 2017;12(2):e0170622.

396. Rogers GB, et al. A novel microbiota stratification system predicts future exacerbations in bronchiectasis. Ann Am Thorac Soc. 2014;11(4):496-503

397. Serisier DJ, et al. Effect of long-term, low-dose erythromycin on pulmonary exacerbations among patients with non-cystic fibrosis bronchiectasis: the BLESS randomized controlled trial. JAMA. 2013;309(12):1260-7.

398. Sulaiman I, et al. Evaluation of the airway microbiome in nontuberculous mycobacteria disease. Eur Respir J, 2018; 52(4). 
399. Macovei L, et al. The hidden 'mycobacteriome' of the human healthy oral cavity and upper respiratory tract. J Oral Microbiol. 2015;7:26094.

400. Javadmoosavi SA, et al. Comparison of the serum concentration of zinc in patients with bronchiectasis and control group. Iran Red Crescent Med J. 2013;15(7):587-9.

401. Beeley JM, Darke CS, Owen GCR. Serum zinc, bronchiectasis, and bronchial carcinoma. Thorax. 1974;29(1):21-5.

402. Lemjabbar-Alaoui $\mathrm{H}$, et al. Lung cancer: biology and treatment options. Biochem Biophys Acta. 2015;1856(2):189-210.

403. van Meerbeeck JP, Fennell DA, De Ruysscher DKM. Small-cell lung cancer. The Lancet. 2011;378(9804):1741-55.

404. Conway EM, et al. Macrophages, inflammation, and lung cancer. 2016, American Thoracic Society. p. 116-130.

405. Mantovani A, et al. Cancer-related inflammation. 2008, Nature Publishing Group. p. 436-444.

406. Lewis CE, Pollard JW. Distinct role of macrophages in different tumor microenvironments. Cancer Res. 2006;66(2):605-17

407. Jiang $L X$, et al. Simultaneous detection of 13 key bacterial respiratory pathogens by combination of multiplex PCR and capillary electrophoresis. Biomed Environ Sci. 2017:30(8):549-61.

408. Greathouse KL, et al. Interaction between the microbiome and TP53 in human lung cancer. Genome Biol. 2018; 19(1).

409. Yan X, et al. Discovery and validation of potential bacterial biomarkers for lung cancer. Am J Cancer Res. 2015;5(10):3111-22.

410. Liu Y, et al. Lung tissue microbial profile in lung cancer is distinct from emphysema. Am J Cancer Res. 2018;8(9):1775-87.

411. Cameron SJS, et al. A pilot study using metagenomic sequencing of the sputum microbiome suggests potential bacterial biomarkers for lung cancer. PLoS ONE, 2017; 12(5).

412. Koshiol J, et al. Helicobacter pylori seropositivity and risk of lung cancer. PLOS ONE. 2012:7(2):32106-32106.

413. Ece $F$, et al. Does Helicobacter pylori infection play a role in lung cancer? Respir Med. 2005;99(10):1258-62.

414. Behroozian R, Moradkhan E. The assessment of probable relationship between lung cancer and Helicobacter pylori infection. Trop Gastroenterol. 2010;31(1):34-6.

415. Samareh-Fekri $M$, et al. Detection of Helicobacter pylori in the bronchoalveolar lavage of patients with lung cancer using real-time PCR. Jundishapur J Microbiol. 2016; 9(11)

416. Xu MY, et al. Association between Helicobacter pylori infection and tumor markers: an observational retrospective study. BMJ Open, 2018; 8(8).

417. Travaglione S, Fabbri A, Fiorentini C. The rho-activating CNF1 toxin from pathogenic E. coli: a risk factor for human cancer development? 2008, Infect Agent Cancer.

418. Yaghoobi $H$, Bandehpour M, Kazemi B. Apoptotic effects of the B subunit of bacterial cytolethal distending toxin on the A549 lung cancer cell line. Asian Pac J Cancer Prev. 2016;17(S3):299-304.

419. Routy B, et al. Gut microbiome influences efficacy of PD-1-based immunotherapy against epithelial tumors. Science. 2018;359(6371):91-7.

420. Callejón-Leblic B, et al. Metal dyshomeostasis based biomarkers of lung cancer using human biofluids. Metallomics. 2018;10(10):1444-51.

421. Zabłocka-Słowińska K, et al. Serum and whole blood Zn, Cu and Mn profiles and their relation to redox status in lung cancer patients. J Trace Elem Med Biol. 2018:45:78-84.

422. Callejón-Leblic B, et al. The metallome of lung cancer and its potential use as biomarker. 2019, MDPI AG.

423. Bai Y, et al. Circulating essential metals and lung cancer: risk assessment and potential molecular effects. Environ Int. 2019;127:685-93.

424. Kuang Y, Wang Q. Iron and lung cancer. Cancer Lett. 2019;464:56-61.

425. Ashmawi SS, Diab HS, Fahmy EA. Evaluation of lung cancer by estimating ferritin in exhaled breath condensate. Egyptian J Chest Dis Tuberculosis. 2015;64(2):465-8.

426. Song B, et al. Down-regulation of lipocalin 2 suppresses the growth of human lung adenocarcinoma through oxidative stress involving Nrf2/ HO-1 signaling. Acta Biochim Biophys Sin. 2015:47(10):805-14.

427. Jiang XP, Elliott RL, Head JF. Manipulation of iron transporter genes results in the suppression of human and mouse mammary adenocarcinomas. Anticancer Res. 2010;30(3):759-65.

428. Yanagawa $\mathrm{H}$, et al. Serum levels of interleukin 6 in patients with lung cancer. Br J Cancer. 1995:71(5):1095-8.
429. Andrews NC. Anemia of inflammation: the cytokine-hepcidin link. 2004 The American Society for Clinical Investigation. p. 1251-1253.

430. Babu KR, Muckenthaler MU. miR-20a regulates expression of the iron exporter ferroportin in lung cancer. J Mol Med. 2016;94(3):347-59.

431. Chanvorachote P, Luanpitpong S. Iron induces cancer stem cells and aggressive phenotypes in human lung cancer cells. Am J Physiol Cell Physiol. 2016;310(9):C728-39.

432. Grivennikov SI, Greten FR, Karin M. Immunity, inflammation, and cancer. 2010, NIH Public Access. p. 883-899.

433. da Silva MC, et al. Iron induces anti-tumor activity in tumor-associated macrophages. Front Immunol. 2017;8(NOV):1479-1479.

434. Vinchi F, et al. Hemopexin therapy reverts heme-induced proinflammatory phenotypic switching of macrophages in a mouse model of sickle cell disease. Blood. 2016;127(4):473-86.

435. Thielmann CM, et al. Iron accumulation in tumor-associated macrophages marks an improved overall survival in patients with lung adenocarcinoma. Sci Rep. 2019; 9(1)

436. Zanganeh $\mathrm{S}$, et al. Iron oxide nanoparticles inhibit tumour growth by inducing pro-inflammatory macrophage polarization in tumour tissues. Nat Nanotechnol. 2016;11(11):986-94.

437. Svensk A-M, et al. Differential expression of superoxide dismutases in lung cancer. Am J Clin Pathol. 2004;122(3):395-404.

438. Yoo DG, et al. Alteration of APE1/ref-1 expression in non-small cell lung cancer: the implications of impaired extracellular superoxide dismutase and catalase antioxidant systems. Lung Cancer. 2008;60(2):277-84.

439. Miar A, et al. Manganese superoxide dismutase (SOD2/MnSOD)/catalase and SOD2/GPX1 ratios as biomarkers for tumor progression and metastasis in prostate, colon, and lung cancer. Free Radic Biol Med. 2015:85:45-55.

440. Zejnilovic J, et al. Association between manganese superoxide dismutase polymorphism and risk of lung cancer. Cancer Genet Cytogenet. 2009;189(1):1-4.

441. Li S, et al. The novel truncated isoform of human manganese superoxide dismutase has a differential role in promoting metastasis of lung cancer cells. Cell Biol Int. 2018;42(8):1030-40.

442. Zhuo H, Smith AH, Steinmaus C. Selenium and lung cancer: a quantitative analysis of heterogeneity in the current epidemiological literature. Cancer Epidemiol Biomarkers Prev. 2004;13(5):771-8.

443. Mahabir S, et al. Dietary zinc, copper and selenium, and risk of lung cancer. Int J Cancer. 2007;120(5):1108-15.

444. Karp DD, et al. Randomized, double-blind, placebo-controlled, phase III chemoprevention trial of selenium supplementation in patients with resected stage I non-small-cell lung cancer: ECOG 5597. J Clin Oncol: Off J Am Soc Clin Oncol. 2013:31(33):4179-87.

445. Idiopathic Pulmonary Fibrosis. Diagnosis and treatment. Am J Respir Crit Care Med. 2000;161(2):646-64.

446. Wuyts WA, et al. Idiopathic pulmonary fibrosis: best practice in monitoring and managing a relentless fibrotic disease. Respiration. 2020:99(1):73-82

447. Paudel KR, et al. Role of lung microbiome in innate immune response associated with chronic lung diseases. Front Med. 2020; 7(554).

448. Song JW, et al. Acute exacerbation of idiopathic pulmonary fibrosis: incidence, risk factors and outcome. Eur Respir J. 2011:37(2):356.

449. Molyneaux PL, et al. The role of bacteria in the pathogenesis and progression of idiopathic pulmonary fibrosis. Am J Respir Crit Care Med. 2014;190(8):906-13.

450. O'Dwyer DN, et al. Lung microbiota contribute to pulmonary inflammation and disease progression in pulmonary fibrosis. Am J Respir Crit Care Med. 2019;199(9):1127-38.

451. Han MK, et al. Lung microbiome and disease progression in idiopathic pulmonary fibrosis: an analysis of the COMET study. Lancet Respir Med. 2014:2(7):548-56

452. Molyneaux PL, et al. Changes in the respiratory microbiome during acute exacerbations of idiopathic pulmonary fibrosis. Respir Res. 2017;18(1):29

453. Huang $Y$, et al. Microbes are associated with host innate immune response in idiopathic pulmonary fibrosis. Am J Respir Crit Care Med. 2017;196(2):208-19.

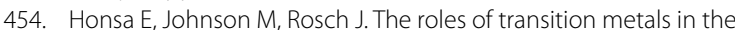
physiology and pathogenesis of Streptococcus pneumoniae. Front Cell Infect Microbiol. 2013; 3(92). 
455. Williams P, Brown MRW. Influence of iron restriction on growth and the expression of outer membrane proteins by Haemophilus influenzae and H. parainfluenzae. FEMS Microbiol Lett. 1986;33(1):153-7.

456. Assad N, et al. Metal-induced pulmonary fibrosis. Curr Environ Health Rep. 2018;5(4):486-98.

457. Bargagli $E$, et al. Analysis of trace elements in bronchoalveolar lavage of patients with diffuse lung diseases. Biol Trace Elem Res. 2008;124(3):225-35.

458. Bargagli $E$, et al. Trace metals in fluids lining the respiratory system of patients with idiopathic pulmonary fibrosis and diffuse lung diseases. J Trace Elem Med Biol. 2017:42:39-44.

459. Kim KH, et al. Iron deposition and increased alveolar septal capillary density in nonfibrotic lung tissue are associated with pulmonary hypertension in idiopathic pulmonary fibrosis. Respir Res. 2010;11(1):37

460. Byrne AJ, Maher TM, Lloyd CM. Pulmonary macrophages: a new therapeutic pathway in fibrosing lung disease? Trends Mol Med. 2016;22(4):303-16.

461. Lee J, et al. Bronchoalveolar lavage (BAL) cells in idiopathic pulmonary fibrosis express a complex pro-inflammatory, pro-repair, angiogenic activation pattern, likely associated with macrophage iron accumulation. PLOS ONE. 2018;13(4):e0194803.

462. Allden SJ, et al. The transferrin receptor CD71 delineates functionally distinct airway macrophage subsets during idiopathic pulmonary fibrosis. Am J Respir Crit Care Med. 2019;200(2):209-19.

463. Sangiuolo $F$, et al. HFE gene variants and iron-induced oxygen radical generation in idiopathic pulmonary fibrosis. Eur Respir $\mathrm{J}$ 2015;45(2):483-90.

464. Wen X, et al. LOXL2, a copper-dependent monoamine oxidase, activates lung fibroblasts through the TGF- $\beta /$ Smad pathway. Int J Mol Med. 2018:42(6):3530-41.

465. Cheng T, et al. Lysyl oxidase promotes bleomycin-induced lung fibrosis through modulating inflammation. J Mol Cell Biol. 2014;6(6):506-15.

466. Aumiller $\mathrm{V}$, et al. Comparative analysis of lysyl oxidase (like) family members in pulmonary fibrosis. Sci Rep. 2017;7(1):149.

467. Barry-Hamilton $V$, et al. Allosteric inhibition of lysyl oxidase-like-2 impedes the development of a pathologic microenvironment. Nat Med. 2010;16(9):1009-17.

468. Mondola P, et al. The $\mathrm{Cu}, \mathrm{Zn}$ superoxide dismutase: not only a dismutase enzyme. Front Physiol. 2016; 7(594).

469. $\mathrm{He} \mathrm{C}$, et al. Mitochondrial $\mathrm{Cu}, \mathrm{Zn}$-superoxide dismutase mediates pulmonary fibrosis by augmenting $\mathrm{H} 2 \mathrm{O} 2$ generation. J Biol Chem. 2011;286(17):15597-607.

470. $\mathrm{He} \mathrm{C}$, et al. $\mathrm{Cu}, \mathrm{Zn}$-superoxide dismutase-mediated redox regulation of jumonji domain containing 3 modulates macrophage polarization and pulmonary fibrosis. Am J Respir Cell Mol Biol. 2016;55(1):58-71.

471. Montani D, et al. Pulmonary arterial hypertension. Orphanet J Rare Dis. 2013:8:97.

472. Farber HW, et al. Five-year outcomes of patients enrolled in the REVEAL registry. Chest. 2015;148(4):1043-54.

473. Tamosiuniene R, Nicolls MR. Regulatory T cells and pulmonary hypertension. Trends Cardiovasc Med. 2011;21(6):166-71.

474. Zhang $C$, et al. Altered airway microbiota composition in patients with pulmonary hypertension. Hypertension. 2020;76(5):1589-99.

475. Kim S, et al. Altered gut microbiome profile in patients with pulmonary arterial hypertension. Hypertension. 2020;75(4):1063-71.

476. Callejo $\mathrm{M}$, et al. Pulmonary arterial hypertension affects the rat gut microbiome. Sci Rep. 2018;8(1):9681.

477. Ruiter $\mathrm{G}$, et al. Iron deficiency is common in idiopathic pulmonary arterial hypertension. Eur Respir J. 2011;37(6):1386-91.

478. Sonnweber T, et al. The crucial impact of iron deficiency definition for the course of precapillary pulmonary hypertension. PLOS ONE. 2018;13(8):e0203396.

479. Haw A, Palevsky HI. Pulmonary hypertension in chronic hemolytic anemias: pathophysiology and treatment. Respir Med. 2018:137:191-200.

480. Niihori M, et al. Rats with a human mutation of NFU1 develop pulmonary hypertension. Am J Respir Cell Mol Biol. 2020;62(2):231-42.

481. Puxeddu $\mathrm{E}$, et al. Iron laden macrophages in idiopathic pulmonary fibrosis: the telltale of occult alveolar hemorrhage? Pulm Pharmacol Ther. 2014:28(1):35-40.
482. Lakhal-Littleton S, et al. Intracellular iron deficiency in pulmonary arterial smooth muscle cells induces pulmonary arterial hypertension in mice. Proc Natl Acad Sci U S A. 2019:116(26):13122-30.

483. Ramakrishnan $\mathrm{L}$, et al. The Hepcidin/Ferroportin axis modulates proliferation of pulmonary artery smooth muscle cells. Sci Rep. 2018;8(1):12972.

484. Ghosh MC, et al. Deletion of iron regulatory protein 1 causes polycythemia and pulmonary hypertension in mice through translational derepression of HIF2a. Cell Metab. 2013;17(2):271-81.

485. Redinus $\mathrm{K}$, et al. An Hb-mediated circulating macrophage contributing to pulmonary vascular remodeling in sickle cell disease. JCI Insight. 2019; 4(15).

486. Zhao $\mathrm{L}$, et al. The zinc transporter ZIP12 regulates the pulmonary vascular response to chronic hypoxia. Nature. 2015;524(7565):356-60.

487. Zimnicka AM, et al. Upregulated copper transporters in hypoxiainduced pulmonary hypertension. PLoS ONE. 2014;9(3):e90544.

488. Sun Q, et al. Selenium and copper as biomarkers for pulmonary arterial hypertension in systemic sclerosis. Nutrients. 2020; 12(6).

489. Bernal PJ, et al. Nitric-oxide-mediated zinc release contributes to hypoxic regulation of pulmonary vascular tone. Circ Res. 2008;102(12):1575-83.

490. White C, et al. Copper transport into the secretory pathway is regulated by oxygen in macrophages. J Cell Sci. 2009;122(Pt 9):1315-21.

491. Poels EM, et al. Supplementing exposure to hypoxia with a copper depleted diet does not exacerbate right ventricular remodeling in mice. PLoS ONE. 2014;9(4):e92983.

492. Zamani Moghaddam AK, et al. Role of selenium from different sources in prevention of pulmonary arterial hypertension syndrome in broiler chickens. Biol Trace Elem Res. 2017;180(1):164-70.

493. Organisation WH. Global Tuberculosis Report 2020. 2020, World Health Organisation

494. Adjemian J, et al. Prevalence of nontuberculous mycobacterial lung disease in U.S. medicare beneficiaries. Am J Respir Critical Care Med. 2012;185(8):881-6.

495. Baldwin SL, et al. The complexities and challenges of preventing and treating nontuberculous mycobacterial diseases. PLoS Negl Trop Dis. 2019;13(2):e0007083.

496. Ehrt S, Schnappinger D, Rhee KY. Metabolic principles of persistence and pathogenicity in Mycobacterium tuberculosis. Nat Rev Microbiol. 2018;16(8):496-507.

497. Douvas GS, May MH, Crowle AJ. Transferrin, iron, and serum lipids enhance or inhibit Mycobacterium avium replication in human macrophages. J Infect Dis. 1993;167(4):857-857.

498. Ratledge C, Dover LG. Iron metabolism in pathogenic bacteria. Annu Rev Microbiol. 2000;54(1):881-941.

499. Ratledge C. Iron, mycobacteria and tuberculosis. Tuberculosis. 2004;84(1-2):110-30.

500. Wong DK, et al. Characterization of exochelins of Mycobacterium avium: evidence for saturated and unsaturated and for acid and ester forms. J Bacteriol. 1996;178(21):6394-8.

501. Parish T, et al. HemZ is essential for heme biosynthesis in Mycobacterium tuberculosis. Tuberculosis. 2005;85(3):197-204.

502. Tullius MV, Nava S, Horwitz MA. PPE37 is essential for Mycobacterium tuberculosis Heme-Iron Acquisition (HIA), and a defective PPE37 in Mycobacterium bovis BCG prevents HIA. Infect Immunity. 2018; 87(2).

503. Owens $C P$, et al. The Mycobacterium tuberculosis secreted protein Rv0203 transfers heme to membrane proteins MmpL3 and MmpL11. J Biol Chem. 2013;288(30):21714-28.

504. Raghu B, Sarma CR, Venkatesan P. Effect of hemoglobin on the growth of mycobacteria and the production of siderophores. Indian J Pathol Microbiol. 1993;36(4):376-82.

505. Rodriguez GM, et al. ideR, An essential gene in mycobacterium tubercuIosis: role of IdeR in iron-dependent gene expression, iron metabolism, and oxidative stress response. Infect Immun. 2002;70(7):3371-81.

506. Reddy PV, et al. Iron storage proteins are essential for the survival and pathogenesis of Mycobacterium tuberculosis in THP-1 macrophages and the guinea pig model of infection. J Bacteriol. 2012;194(3):567-75.

507. Pandey R, Rodriguez GM. A ferritin mutant of Mycobacterium tuberculosis is highly susceptible to killing by antibiotics and is unable to establish a chronic infection in mice. Infect Immun. 2012;80(10):3650-9. 
508. Janowski R, Auerbach-Nevo T, Weiss MS. Bacterioferritin from Mycobacterium smegmatis contains zinc in its di-nuclear site. Protein Sci. 2008;17(7):1138-50

509. Namasivayam $\mathrm{S}$, et al. The microbiome and tuberculosis: early evidence for cross talk. mBio. 2018; 9(5).

510. Cui Z, et al. Complex sputum microbial composition in patients with pulmonary tuberculosis. BMC Microbiol. 2012;12(1):276

511. Zhou Y, et al. Correlation between either cupriavidus or porphyromonas and primary pulmonary tuberculosis found by analysing the microbiota in patients' bronchoalveolar lavage fluid. PLOS ONE. 2015;10(5):e0124194.

512. Segal LN, et al. Anaerobic bacterial fermentation products increase tuberculosis risk in antiretroviral-drug-treated HIV patients. Cell Host Microbe. 2017;21(4):530-537.e4.

513. Winglee $K$, et al. Aerosol Mycobacterium tuberculosis infection causes rapid loss of diversity in gut microbiota. PLoS ONE. 2014;9(5):e97048,

514. Namasivayam, S., et al., Longitudinal profiling reveals a persistent intestinal dysbiosis triggered by conventional anti-tuberculosis therapy. Microbiome, 2017. 5(1)

515. Hu Y. et al. The gut microbiome signatures discriminate healthy from pulmonary tuberculosis patients. Front Cell Infect Microbiol. 2019; 9.

516. Boradia VM, et al. Mycobacterium tuberculosis acquires iron by cellsurface sequestration and internalization of human holo-transferrin. Nat Commun. 2014;5(1):4730.

517. Abel L, et al. Susceptibility to leprosy is linked to the human NRAMP1 gene. J Infect Dis. 1998;177(1):133-45.

518. Bellamy R, et al. Variations in the NRAMP1 gene and susceptibility to tuberculosis in West Africans. N Engl J Med. 1998;338(10):640-4.

519. Souza KS, et al. Simple nucleotide polymorphisms in the NRAMP1 / SLC11A1 gene to the risk of susceptibility to tuberculosis. Res, Soc Dev. 2020:9(11):e2489119708.

520. Cellier M, et al. Expression of the human NRAMP1 gene in professional primary phagocytes: studies in blood cells and in $\mathrm{HL}-60$ promyelocytic leukemia. J Leukoc Biol. 1997;61(1):96-105.

521. Searle S, et al. Localisation of Nramp1 in macrophages: modulation with activation and infection. J Cell Sci. 1998;111(Pt 19):2855-66.

522. Vidal SM, et al. Natural resistance to intracellular infections: Nramp1 encodes a membrane phosphoglycoprotein absent in macrophages from susceptible (Nramp1 D169) mouse strains. J Immunol. 1996:157(8):3559-68.

523. Cellier MF. Nramp: from sequence to structure and mechanism of divalent metal import. Curr Top Membr. 2012;69:249-93.

524. Kuhn DE, et al. Differential iron transport into phagosomes isolated from the RAW264.7 macrophage cell lines transfected with Nramp1Gly169 or Nramp1Asp169. J Leukoc Biol. 1999;66(1):113-9.

525. Zwilling BS, et al. Role of iron in Nramp1-mediated inhibition of mycobacterial growth. Infect Immun. 1999;67(3):1386-92.

526. Barton $\mathrm{CH}$, et al. Nramp1: a link between intracellular iron transport and innate resistance to intracellular pathogens. J Leukoc Biol. 1999;66(5):757-62.

527. Cunrath O, Bumann D. Host resistance factor SLC11A1 restricts Salmonella growth through magnesium deprivation. Science. 2019:366(6468):995-9.

528. Fritsche $\mathrm{G}$, et al. Nramp1 functionality increases inducible nitric oxide synthase transcription via stimulation of IFN regulatory factor 1 expression. J Immunol. 2003;171(4):1994-8.

529. Fritsche G, et al. Slc1 1a1 (Nramp1) impairs growth of Salmonella enterica serovar typhimurium in macrophages via stimulation of lipocalin-2 expression. J Leukoc Biol. 2012;92(2):353-9.

530. Frehel C, et al. Effect of Nramp1 on bacterial replication and on maturation of Mycobacterium avium-containing phagosomes in bone marrowderived mouse macrophages. Cell Microbiol. 2002:4(8):541-56.

531. North RJ, et al. Consequence of Nramp1 deletion to Mycobacterium tuberculosis infection in mice. Infect Immun. 1999;67(11):5811-4.

532. Gomes and Appelberg. Evidence for a link between iron metabolism and Nramp1 gene function in innate resistance against Mycobacterium avium. Immunology. 1998;95(2):165-8.

533. Lafuse WP, Alvarez GR, Zwilling BS. Regulation of Nramp1 mRNA stability by oxidants and protein kinase C in RAW264.7 macrophages expressing Nramp1(Gly169). Biochem J. 2000;351 pt 3:687-96.
534. Silva-Gomes $\mathrm{S}$, et al. Mycobacterium avium infection induces $\mathrm{H}$-ferritin expression in mouse primary macrophages by activating toll-like receptor 2. PLoS ONE. 2013:8(12):e82874.

535. Phelan JJ, et al. Desferrioxamine supports metabolic function in primary human macrophages infected with Mycobacterium tuberculosis. Front Immunol. 2020;11:836.

536. Braverman J, et al. HIF-1a is an essential mediator of IFN- $y$ dependent immunity to Mycobacterium tuberculosis. J Immunol. 2016:197(4):1287-97.

537. Halaas $\varnothing$, et al. Intracellular Mycobacterium avium intersect transferrin in the Rab11+recycling endocytic pathway and avoid lipocalin 2 trafficking to the lysosomal pathway. J Infect Dis. 2010;201(5):783-92.

538. Kelley VA, Schorey JS. Mycobacterium's arrest of phagosome maturation in macrophages requires Rab5 activity and accessibility to iron. Mol Biol Cell. 2003;14(8):3366-77.

539. Ward SK, Hoye EA, Talaat AM. The global responses of Mycobacterium tuberculosis to physiological levels of copper. J Bacteriol. 2008;190(8):2939-46.

540. Speer A, et al. Porins increase copper susceptibility of Mycobacterium tuberculosis. J Bacteriol. 2013;195(22):5133-40.

541. Wolschendorf F, et al. Copper resistance is essential for virulence of Mycobacterium tuberculosis. Proc Natl Acad Sci U S A. 2011;108(4):1621-6.

542. Ward SK, et al. CtpV: a putative copper exporter required for full virulence of Mycobacterium tuberculosis. Mol Microbiol. 2010;77(5):1096-110.

543. Liu T, et al. CsoR is a novel Mycobacterium tuberculosis copper-sensing transcriptional regulator. Nat Chem Biol. 2007;3(1):60-8.

544. Festa RA, et al. A novel copper-responsive regulon in Mycobacterium tuberculosis. Mol Microbiol. 2011;79(1):133-48.

545. Gold B, et al. Identification of a copper-binding metallothionein in pathogenic mycobacteria. Nat Chem Biol. 2008;4(10):609-16.

546. Shi X. et al. The copper-responsive RicR regulon contributes to Mycobacterium tuberculosis virulence. mBio. 2014. 5(1).

547. Harris AB. Inhibition of growth and nucleic acid synthesis in zinc-deficient Mycobacterium smegmatis. J Gen Microbiol. 1969;56(1):27-33.

548. Dow A, Prisic S. Alternative ribosomal proteins are required for growth and morphogenesis of Mycobacterium smegmatis under zinc limiting conditions. PLoS ONE. 2018;13(4):e0196300.

549. Eckelt $\mathrm{E}$, et al. Identification of a lineage specific zinc responsive genomic island in Mycobacterium avium ssp. paratuberculosis. BMC Genomics. 2014:15(1):1076.

550. Tinaztepe $E$, et al. Role of metal-dependent regulation of ESX-3 secretion in intracellular survival of Mycobacterium tuberculosis. Infect Immun. 2016;84(8):2255-63.

551. Riccardi G, et al. Genomic analysis of zinc homeostasis in Mycobacterium tuberculosis. FEMS Microbiol Lett. 2008;287(1):1-7.

552. Gopal R, et al. S100A8/A9 proteins mediate neutrophilic inflammation and lung pathology during tuberculosis. Am J Respir Crit Care Med. 2013;188(9):1137-46.

553. LiY, et al. Zinc depletion induces ribosome hibernation in mycobacteria. Proc Natl Acad Sci U S A. 2018;115(32):8191-6.

554. LiY, et al. Progression from remodeling to hibernation of ribosomes in zinc-starved mycobacteria. Proc Natl Acad Sci U S A. 2020;117(32):19528-37.

555. Padilla-Benavides T, et al. A novel P(1B)-type Mn2+-transporting ATPase is required for secreted protein metallation in mycobacteria. J Biol Chem. 2013;288(16):11334-47.

556. Zambelli B, et al. Biochemical studies on Mycobacterium tuberculosis UreG and comparative modeling reveal structural and functional conservation among the bacterial UreG family. Biochemistry. 2007:46(11):3171-82.

557. Habel JE, et al. Structure of Rv1848 (UreA), the Mycobacterium tuberculosis urease gamma subunit. Acta Crystallogr Sect F Struct Biol Cryst Commun. 2010;66(Pt 7):781-6.

558. Savvi $\mathrm{S}$, et al. Functional characterization of a vitamin B12-dependent methylmalonyl pathway in Mycobacterium tuberculosis: implications for propionate metabolism during growth on fatty acids. J Bacteriol. 2008;190(11):3886-95.

559. Warner DF, et al. A riboswitch regulates expression of the coenzyme B12-independent methionine synthase in Mycobacterium tuberculosis: 
implications for differential methionine synthase function in strains H37Rv and CDC1551. J Bacteriol. 2007;189(9):3655-9.

560. Rodionov DA, et al. Comparative genomics of the vitamin B12 metabolism and regulation in prokaryotes. J Biol Chem. 2003;278(42):41148-59.

561. Lisher JP, Giedroc DP. Manganese acquisition and homeostasis at the host-pathogen interface. Front Cell Infect Microbiol. 2013;3:91.

562. Yatmark $P$, et al. Effects of iron chelators on pulmonary iron overload and oxidative stress in $\beta$-thalassemic mice. Pharmacology. 2015:96(3-4):192-9.

563. Sohn YS, et al. Redistribution of accumulated cell iron: a modality of chelation with therapeutic implications. Blood. 2008;111(3):1690-9.

564. Zhou T, et al. Design of iron chelators with therapeutic application. Dalton Trans. 2012:41(21):6371-89.

565. Choi SR, et al. In vitro efficacy of free and nanoparticle formulations of gallium(III) meso-tetraphenylporphyrine against Mycobacterium avium and mycobacterium abscessus and gallium biodistribution in mice. Mol Pharm. 2018;15(3):1215-25.

566. Choi SR, et al. Gallium porphyrin and gallium nitrate synergistically inhibit mycobacterial species by targeting different aspects of iron/ heme metabolism. ACS Infect Dis. 2020;6(10):2582-91.

567. Di Renzo L, et al. COVID-19: is there a role for immunonutrition in obese patient? J Trans Med. 2020; 18(1).

\section{Publisher's Note}

Springer Nature remains neutral with regard to jurisdictional claims in published maps and institutional affiliations.
Ready to submit your research? Choose BMC and benefit from:

- fast, convenient online submission

- thorough peer review by experienced researchers in your field

- rapid publication on acceptance

- support for research data, including large and complex data types

- gold Open Access which fosters wider collaboration and increased citations

- maximum visibility for your research: over $100 \mathrm{M}$ website views per year

At BMC, research is always in progress.

Learn more biomedcentral.com/submissions 